\title{
Modulation of Coronary Reactive Hyperemia through Soluble Epoxide Hydrolase: Role of Oxylipins and PPARgamma
}

\author{
Ahmad Mahmoud Hanif
}

Follow this and additional works at: https://researchrepository.wvu.edu/etd

\section{Recommended Citation}

Hanif, Ahmad Mahmoud, "Modulation of Coronary Reactive Hyperemia through Soluble Epoxide Hydrolase: Role of Oxylipins and PPARgamma" (2016). Graduate Theses, Dissertations, and Problem Reports. 5754.

https://researchrepository.wvu.edu/etd/5754

This Dissertation is protected by copyright and/or related rights. It has been brought to you by the The Research Repository @ WVU with permission from the rights-holder(s). You are free to use this Dissertation in any way that is permitted by the copyright and related rights legislation that applies to your use. For other uses you must obtain permission from the rights-holder(s) directly, unless additional rights are indicated by a Creative Commons license in the record and/ or on the work itself. This Dissertation has been accepted for inclusion in WVU Graduate Theses, Dissertations, and Problem Reports collection by an authorized administrator of The Research Repository @ WVU.

For more information, please contact researchrepository@mail.wvu.edu. 


\title{
Modulation of Coronary Reactive Hyperemia through Soluble Epoxide Hydrolase: Role of Oxylipins and PPAR $\gamma$
}

\author{
Ahmad Mahmoud Hanif \\ Dissertation submitted to the School of Pharmacy at West Virginia University in partial \\ fulfillment of the requirements for the degree of \\ Doctor of Philosophy $(\mathrm{PhD})$ in \\ Pharmaceutical and Pharmacological Sciences
} Mohammed A. Nayeem, PhD, Chair

S. Jamal Mustafa, PhD Kan Hong, $\mathrm{PhD}$ Jason Huber, PhD Grazyna Szklarz, PhD Morgantown, West Virginia, USA Department of Pharmaceutical and Pharmacological Sciences 2016

C Copyrights by Ahmad Hanif, 2016

All Rights Reserved 


\begin{abstract}
Modulation of Coronary Reactive Hyperemia through Soluble Epoxide
\end{abstract}

Hydrolase: Role of Oxylipins and PPAR $\gamma$

Ahmad Mahmoud Hanif

Ischemic heart disease (IHD) continues to be a leading cause of morbidity and mortality in increasingly more countries. Function and structural damage to the heart are likely consequences of ischemic insults and may even lead to death. The body's physiological response to ischemia is by increasing nourishment or perfusion to the deprived organ in an attempt to decrease the potential ischemia-induced damage and achieve faster recovery. This reaction to ischemia is termed reactive hyperemia $(\mathrm{RH})$, and in the heart, it is coronary reactive hyperemia $(\mathrm{CRH})$. Epoxyeicosatrienoic acids (EETs) are oxylipins derived from arachidonic acid (AA) and possess distinct cardiovascular benefits, such as vasodilation, cardio-protection in ischemia/reperfusion, and anti-inflammation. Other oxylipins, such as HETEs, epoxyoctadecaenoic acid (EpOMEs), HODEs, and prostanoids have versatile and yet incompletely elucidated cardiovascular effects. Moreover, these oxylipins seem to be interconnected and can affect each other. For example, the enzyme soluble epoxide hydrolase $(\mathrm{sEH})$ is involved in the main catabolic pathway of EETs, which are converted into dihydroxyeicosatrienoic acids (DHETs), and EpOMEs which are converted into dihydroxyoctadecaenoic acids (DiHOMEs). The relationship among sEH, CYPepoxygenase pathway, oxylipins, PPAR $\gamma$, and CRH response to a brief ischemia is not known. We hypothesized that targeting $\mathrm{sEH}$, by pharmacologic inhibition or genetic deletion, enhances $\mathrm{CRH}$ in isolated mouse hearts through changing the oxylipin profiles, including an increase in EETs/DHETs ratio. Targeting sEH resulted in significant enhancement of CRH, including repayment volume, repayment duration, and repayment/debt ratio $(\mathrm{p} \leq 0.05)$. Also, oxylipin profiles were changed because of sEH targeting, including increase in EET/DHET ratio, increase in EpOME/DiHOME ratio, increase in the level of HODEs, decrease in the levels of mid-chain HETEs, and decrease in prostanoids $(\mathrm{p} \leq 0.05)$. Involvement of PPAR $\gamma$ in the modulation of CRH was demonstrated using a PPAR $\gamma$-antagonist (T0070907) and a PPAR $\gamma$-agonist (rosiglitazone). T0070907 reduced CRH ( $\mathrm{p} \leq 0.05)$, whereas, rosiglitazone enhanced $\mathrm{CRH}(\mathrm{p} \leq$ $0.05)$ in isolated mouse hearts compared to the non-treated.

These data demonstrate that sEH disruption enhances CRH possibly through causing changes in oxylipin profiles. Also, PPAR $\gamma$ mediate $\mathrm{CRH}$ downstream of the CYP epoxygenases-EET pathway. 
West Virginia University Electronic

Thesis and Dissertation Signature

Student Name: $\frac{\text { HANIF }}{\text { (Last) }}$ (First)

Student ID : $701-185-924 \quad$ Non-Wvi Email Account:apmitigmail.com

Degree:

Master's $\quad \times$ Doctorate

Document Type:

Thesis $\quad X \quad$ Dissertation

Document Title: Modulation of Coronary Reactive Hoperemia

\section{Student Agreement:}

I hereby certify that, if appropriate, I have obtained and attached hereto a written permission statement from the owners of each third party copyrighted matter to be included in my thesis, dissertation, project report, or other research material, allowing distribution as specified upon deposit.

I hereby grant to West Virginia University and its agents the non-exclusive license to archive and make accessible, under the conditions selected upon deposit, my above mentioned document in whole or in part in all forms of media, now or hereafter known. I retain ownership rights as specified in the WVU copyright policy to the copyright of the abovementioned document. I also retain the right to use in future works (such as articles or books) all or part of this abovementioned document.

\section{Review and Acceptance:}

The above mentioned document has been reviewed and accepted by the student's advisory committee. The undersigned agree to abide by the statements above, and agree that this Signature Form updates any and all previous Signature Forms submitted heretofore.

Signed:

Committee:
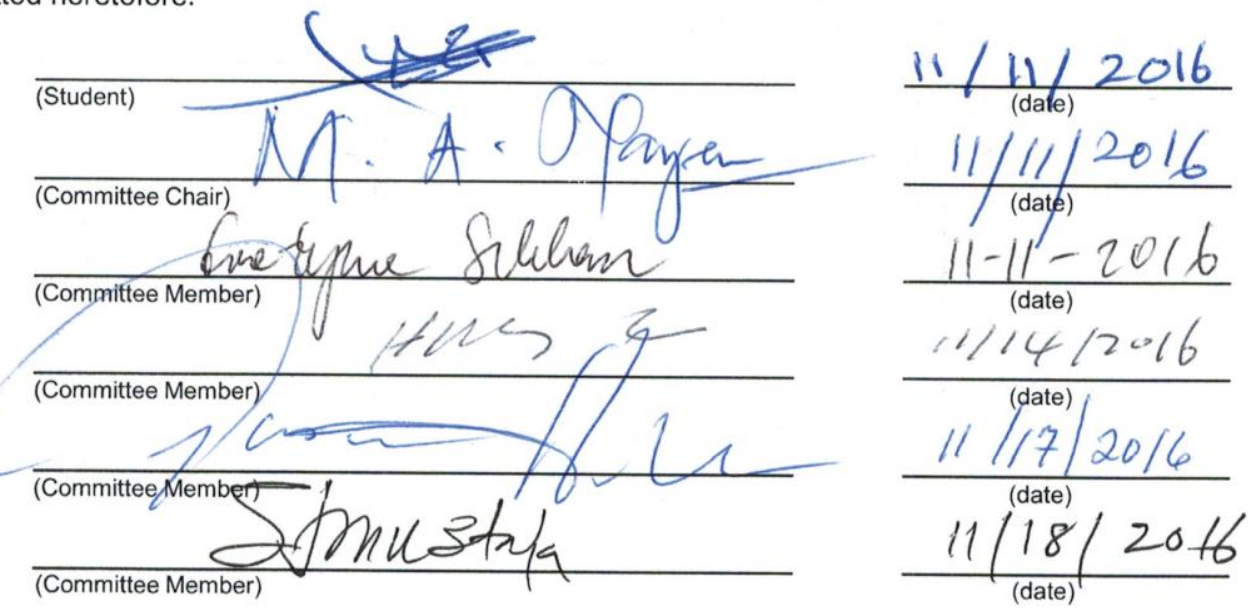

$\frac{1114 / 2016}{\text { (date) }}$

$\frac{1 / \int^{\text {(date) }} / 20 / 6}{\text { (date) }}$

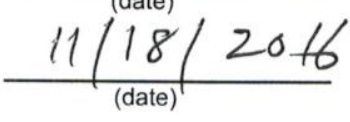

(Committee Member) 


\section{DEDICATIONS}

To my parents, my wife, and my family

I am dedicating my work to my parents: my father Mahmoud Hanif and my mother Mariam Abu Moailish, for unconditionally believing in me, supporting me, and keeping me in their prayers to achieve my aspirations. Despite the bitterness of having their son distantly far away from them, they made the sacrifice to help me fulfill my dream of pursuing graduate studies.

I am also dedicating this work to my beloved wife, Kholoud Alhindi, for all the support and encouragement, for believing in me, and, most importantly, for standing by my side during the financially tight five years of the graduate study. I would not have been able to achieve this goal if it were not for her support and dedication.

Finally, I am dedicating my work to my children: Shahd, Yazan, Zaid, Rayyan, and Kenan, and to my siblings: Ghada, Ayman, Wafa'a, Muhanned, Moayyad, Eman, and Mohammed. 


\section{ACKNOWLEDGEMENTS}

I would like to sincerely thank everyone who supported me through the inception, development, and completion of this dissertation. I will be forever indebted and grateful to Dr. Mohammed Nayeem, my mentor and committee chair. His insight, encouragement, guidance, and patience have made this dissertation possible. I would like to thank my dissertation committee members, Dr. Jamal Mustafa, Dr. Kan Hong, Dr. Jason Huber, and Dr. Grazyna Szklarz for their advice and suggestions at various stages of preparing and writing this dissertation. I am greatly indebted to Dr. Bunyen Teng, and Dr. Xueping Zhou, for teaching me and helping me understand, with matchless patience, how to perform and trouble-shoot the complex technique of Isolated Prefused Heart. They both have embraced me as a colleague from day one and relentlessly encouraged me throughout the practice and experimentation periods. I would also like to thank my friends and colleagues from Dr. Nayeem's and Dr. Mustafa's laboratories: Dr. Isha Pradhan, Dr. Dovenia Pannoth, Dr. Hicham Labazi, Dr. Vishal Yadav, and Mrs. Sherry Xie for their help, support and advice. Additionally, I would like to thank my teachers during the didactic part of the program; their efforts were pivotal to expanding my knowledge and to be better equipped for the requirements of the program.

Finally, I would like the institutions and departments, whose support was pivotal in fulfilling this project: the Graduate Program at West Virginia University, specifically Dr. Fred Minnear and Dr. Jason Huber, for believing in me and for giving me the chance to be a graduate student in their program; National Institutes of Health Grant (HL-114559) to M. A. Nayeem for financially supporting me, providing the chemicals and other costs, and for sponsoring me to attend different 
conferences in related fields; the Office of Laboratory Animals Resources at West Virginia University for their guidance and support with animal breeding and care; the School of Pharmacy at West Virginia University and the American Society for Pharmacology and Experimental Therapeutics for the financial support, which allowed me to attend relevant conferences. 
DEDICATION iv

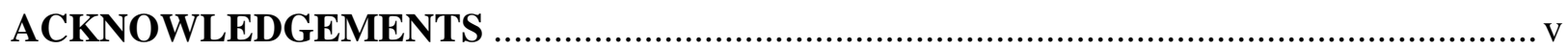

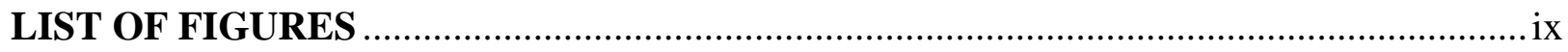

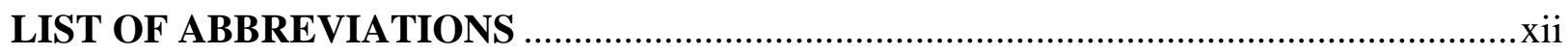

INTRODUCTION



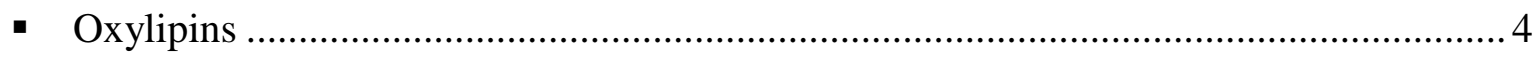

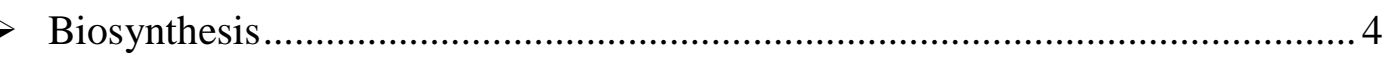

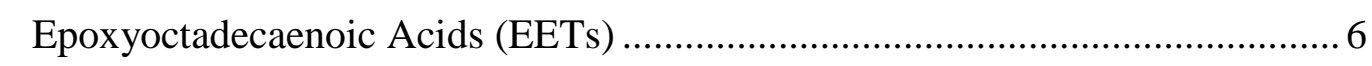

> Mid-chain Hydroxyeicosatetraenoic Acids (HETEs) ..........................................

$>$ Epoxyeicosatrienoic Acids (EpOMEs) ......................................................... 8

$>$ Hydroxyoctadecadienoic Acids (HODEs) …………....................................... 8

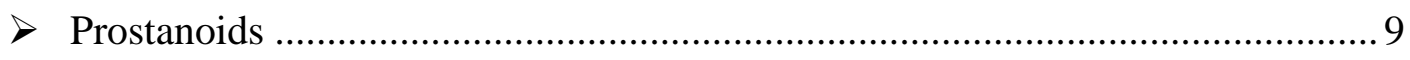

- Coronary Reactive Hyperemia in the Isolated Perfused Heart........................................ 11

- Peroxisome Proliferator-Activated Receptors (PPARs) .................................................. 14

- Hypothesis and Project Summary …………………………...................................... 15

CHAPTER ONE: Deletion of Soluble Epoxide Hydrolase Enhances Coronary Reactive

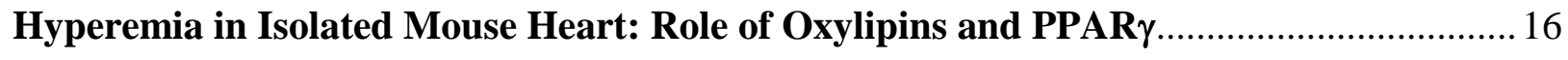

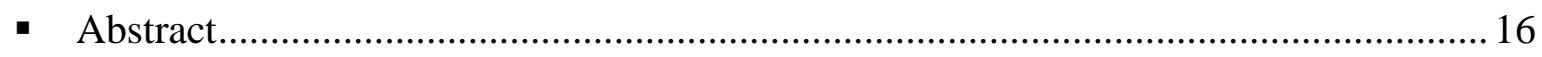

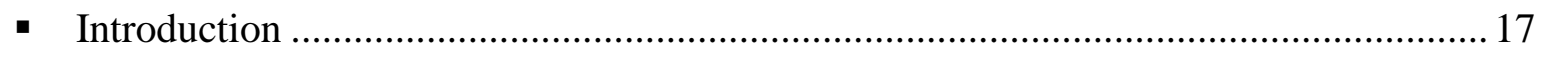

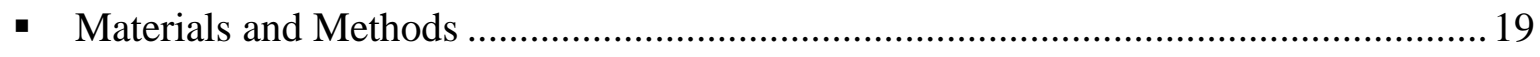




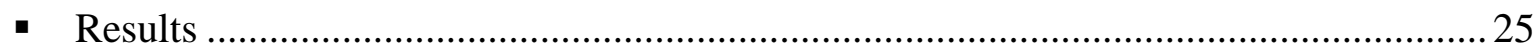

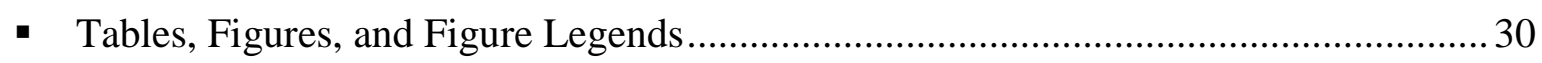

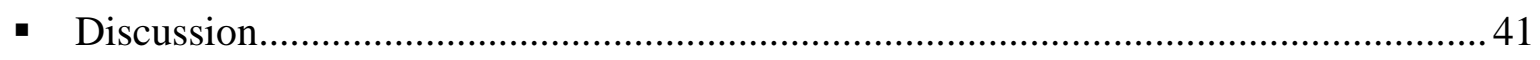

- Perspective and Significance ............................................................................ 47

CHAPTER TWO: Effect of Soluble Epoxide Hydrolase on the Modulation of Coronary

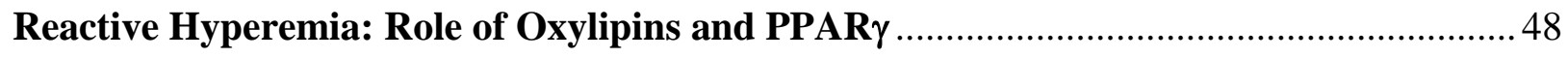

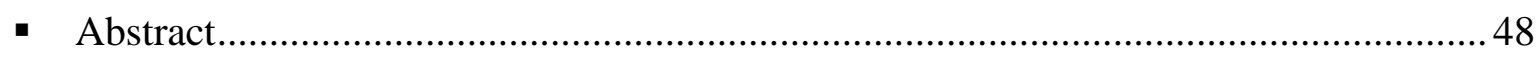

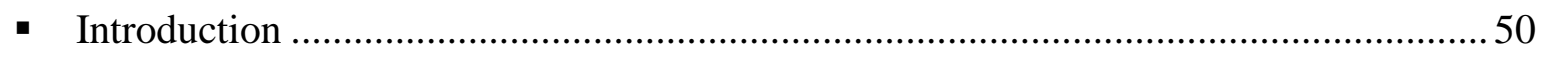

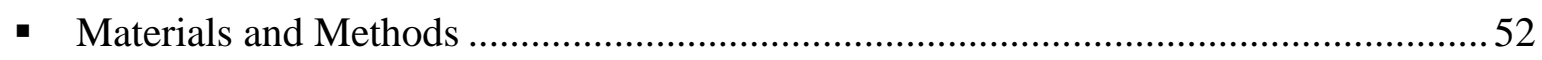

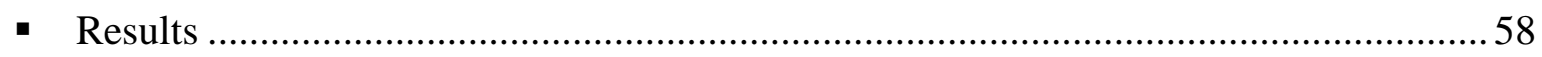

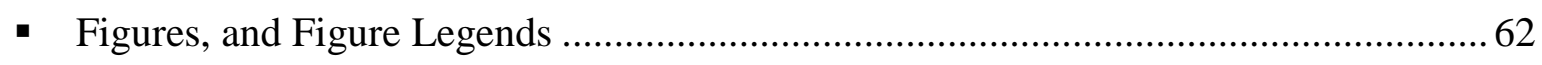

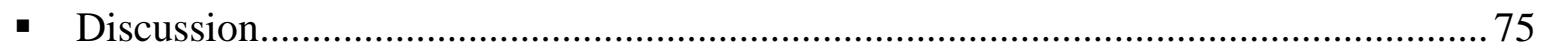

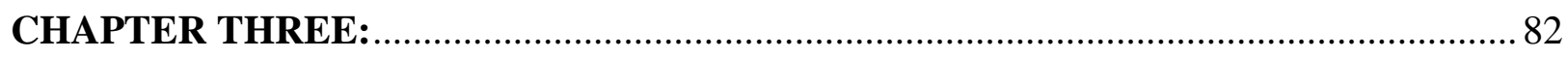

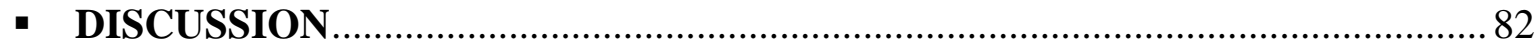

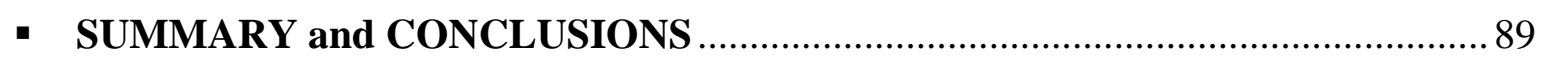

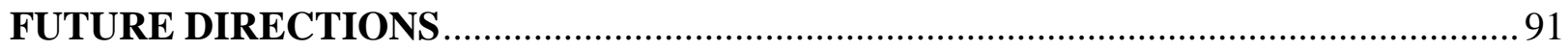

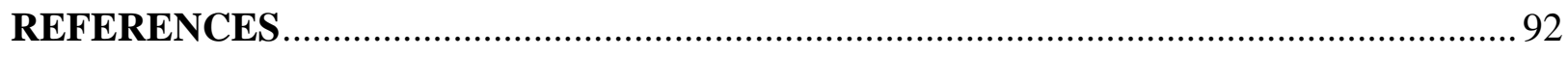

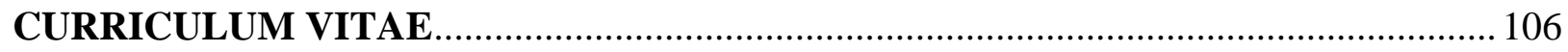




\section{LIST OF FIGURES}

Figure

Page

Figure I: Parameters derived from coronary reactive hyperemia (CRH) before and after 15-sec ischemia 3

Figure II: Oxylipins derived from arachidonic and linoleic acids. .10

Figure III: Schematic picture showing the setup of Langendorff perfused heart experiment .....13

Figure 1.1: Repayment volume in female and male $\mathrm{sEH}^{+/+}$and $\mathrm{sEH}^{-/-}$mice. 31

Figure 1.2: Comparison of coronary reactive hyperemia between $\mathrm{sEH}^{+/+}$and $\mathrm{sEH}^{-/}$mice .32

Figure 1.3: LC-MS/MS analysis of 14,15-EET and 14,15-DHET in $\mathrm{sEH}^{+/+}$and $\mathrm{sEH}^{-/-}$mice heart perfusate at baseline and post ischemia .33

Figure 1.4: $\mathrm{LC}-\mathrm{MS} / \mathrm{MS}$ analysis of mid-chain HETEs in $\mathrm{sEH}^{+/+}$and $\mathrm{sEH}^{-/}$mice heart perfusate at baseline and post ischemia. .34

Figure 1.5: LC-MS/MS analysis of EpOMEs and DiHOMEs in $\mathrm{sEH}^{+/+}$and $\mathrm{sEH}^{-/}$mice heart perfusate at baseline and post ischemia .35

Figure 1.6: $\mathrm{LC}-\mathrm{MS} / \mathrm{MS}$ analysis of $\mathrm{HODEs}$ in $\mathrm{sEH}^{+/+}$and $\mathrm{sEH}^{-/-}$mice heart perfusate at baseline and post ischemia. .36 
Figure 1.7: Comparison of the effect of PPAR $\gamma$-antagonist, T0070907, $(10 \mu \mathrm{M})$ on coronary reactive hyperemia between $\mathrm{sEH}^{+/+}$and $\mathrm{sEH}^{-/-}$mice

Figure 1.8: Comparison of the effect of PPAR $\gamma$-agonist, rosiglitazone, $(10 \mu \mathrm{M})$ on coronary reactive hyperemia between $\mathrm{sEH}^{+/+}$and $\mathrm{sEH}^{-/-}$mice .38

Figure 1.9: Comparison of the effect of L-NAME $(100 \mu \mathrm{M})$ on coronary reactive hyperemia between $\mathrm{sEH}^{+/+}$and $\mathrm{sEH}^{-1-}$ mice.

Figure 1.10: A schematic diagram summarizing oxylipin changes in response to short-lived ischemia in $\mathrm{sEH}^{+/+}$and $\mathrm{sEH}^{-/-}$mice heart perfusate

Figure 2.1: Comparison of coronary reactive hyperemia between WT and $t$-AUCB-treated WT mice. .63

Figure 2.2: Effect of $t$-AUCB, soluble epoxide hydrolase-inhibitor, on coronary reactive hyperemia in $\mathrm{sEH}^{-/-}$mice .64

Figure 2.3: LC-MS/MS analysis of 14,15-EET, 11,12- and 14,15-DHETs in WT and $t$-AUCBtreated WT mice heart perfusate at baseline and post ischemia .65

Figure 2.4: LC-MS/MS analysis of mid-chain HETEs in WT and $t$-AUCB-treated WT mice heart perfusate at baseline and post ischemia

Figure 2.5: LC-MS/MS analysis of EpOMEs and DiHOMEs in WT and $t$-AUCB-treated WT mice heart perfusate at baseline and post ischemia 
Figure 2.6: LC-MS/MS analysis of HODEs in WT and $t$-AUCB-treated WT mice heart perfusate at baseline and post ischemia .68

Figure 2.7: LC-MS/MS analysis of prostanoids in WT and $t$-AUCB-treated WT mice heart perfusate at baseline and post ischemia

Figure 2.8: Comparison of the effect of CYP-epoxygenase inhibitor, MS-PPOH, $(1 \mu \mathrm{M})$ on coronary reactive hyperemia between WT and $t$-AUCB-treated WT mice. .70

Figure 2.9: Comparison of the effect of PPAR $\gamma$-antagonist, T0070907, $(10 \mu \mathrm{M})$ on coronary reactive hyperemia in $t$-AUCB-treated WT mice after 15 -sec ischemia .71

Figure 2.10: Effect of PPAR $\gamma$-antagonist, T0070907, $(10 \mu \mathrm{M})$ on coronary reactive hyperemia between WT and $t$-AUCB-treated WT mice .72

Figure 2.11: Comparison of the effect of PPAR $\gamma$-agonist, rosiglitazone, $(10 \mu \mathrm{M})$ on coronary reactive hyperemia between $\mathrm{WT}$ and $t$-AUCB-treated WT mice. .73

Figure 2.12: A schematic diagram summarizing oxylipin changes in response to short-lived ischemia in WT and $t$-AUCB-treated WT mice heart perfusate. .74 


\section{LIST OF ABBREVIATIONS}

\begin{tabular}{|c|c|}
\hline$\mu \mathrm{g}:$ & micro gram \\
\hline$\mu \mathrm{l}:$ & micro liter \\
\hline 14,15 EEZE: & 14,15-epoxyeicosa-5(z)-enoic \\
\hline $6 \mathrm{~K}-\mathrm{PG}-\mathrm{F}_{1 \alpha}:$ & 6-keto prostaglandin- $\mathrm{F}_{1 \alpha}$ \\
\hline AA: & Arachinodic acid \\
\hline ANOVA: & Analysis of variance \\
\hline AUDA: & 12-(3-adamantan-1-yl-ureido)-dodecanoic acid \\
\hline $\mathrm{A}_{2 \mathrm{~A}} \mathrm{AR}$ : & $\mathrm{A}_{2 \mathrm{~A}}$ adenosine receptor \\
\hline $\mathrm{A}_{2 \mathrm{~B}} \mathrm{AR}:$ & $\mathrm{A}_{2 \mathrm{~B}}$ adenosine receptor \\
\hline BK: & Large conductance potassium channels \\
\hline BP & Blood Pressure \\
\hline $\mathrm{Ca}^{2+}:$ & Calcium \\
\hline cAMP: & Cyclic adenosine monophosphate \\
\hline COX: & Cyclooxygenase \\
\hline CRH: & coronary reactive hyperemia \\
\hline CYP2J2: & Cytochrome P450 2J2 \\
\hline Cyp450: & Cytochrome P450 \\
\hline DDMS: & dibromo-dodecenyl-methylsulfimide \\
\hline DHETs: & dihydroxyeicosatrienoic acids \\
\hline DiHOMEs: & dihydroxyoctadecaenoic acids \\
\hline DMSO: & Dimethyl sulfoxide \\
\hline DOCA & Deoxycorticosterone acetate \\
\hline
\end{tabular}


EDHF:

EETs:

EpOMEs:

GPCR:

HETEs:

HODEs:

HR:

$\mathrm{IP}_{3}$ :

$\mathrm{K}_{\text {ATP: }}$

$\mathrm{K}_{\mathrm{Ca}}$ :

$\mathrm{Kg}$ :

LC-MS/MS:

LOX:

LVDP:

$\mathrm{mEH}$ :

mg:

$\mathrm{ml}$ :

$\mathrm{mm}$ :

$\mathrm{mM}$ :

MS-PPOH:

NFкB:

NO:

PG:
Endothelium-Derived Hyperpolarizing Factor

Epoxyeicosatrienoic acids

epoxyoctadecaenoic acids

G Protein Coupled Receptor

hydroxyeicosatetraenoic acids

hydroxyoctadecadienoic acids

heart rate

Inositol triphosphate

ATP-sensitive $\mathrm{K}^{+}$channels

Calcium-acitvated $\mathrm{K}^{+}$channels

Kilogram

liquid chromatography, tandem mass spectroscopy

Lipoxygenase

left ventricular developed pressure

Microsomal epoxide hydrolase

milligram

milliliter

millimeter

millimole

methylsulfonyl-propargyloxyphenylhexanamide

Nuclear factor kappa light chain enhancer of activated B cells

Nitric oxide

prostaglandin 
PHF:

PKA:

PKC:

$\mathrm{PLA}_{2}$ :

PLC:

PPAR:

PPAR $\gamma:$

PUFAs:

R/D:

$\mathrm{RD}$ :

$\mathrm{RV}$ :

SAH:

sEH:

$\mathrm{sEH}^{-/-}$:

$\mathrm{sEH}^{+/+}$:

SEM:

t-AUCB:

TxA

$\mathrm{TxB}_{2}$ : peak hyperemic flow

Protein kinase A

Protein kinase C

Phospholipase $\mathrm{A}_{2}$

Phospholipase C

Peroxisome proliferator-activated receptor

peroxisome proliferator-activated receptor - gamma

polyunsaturated fatty acids

repayment/debt ratio

repayment duration

repayment volume

S-Adenosyl-L-homocysteine

soluble epoxide hydrolase

sEH-knockout mice

Wild type mice normally expressing sEH

Standard error of the mean

trans-4-[4-(3-adamantan-1-ylureido)cyclohexyloxy] benzoic acid

thromboxane $\mathrm{A}_{2}$

thromboxane $\mathrm{B}_{2}$ 


\section{INTRODUCTION}

\section{Reactive Hyperemia}

When the heart is subjected to a brief period of ischemia it responds by momentarily increasing coronary blood flow (12), which is known as reactive hyperemia (RH) or Coronary RH (CRH). The phenomenon of $\mathrm{CRH}$ is associated with significant coronary vasodilation in response to cessation in coronary perfusion. This phenomenon is not unique to the heart; it is observed in other tissues when they are temporarily deprived of blood perfusion. This occlusion, or ischemia, is inherently associated with potentially detrimental effects on the heart and cardiac function. The increase in blood flow coupled with $\mathrm{CRH}$ is known as repayment volume; it aims at preventing or lessening the potential injury due to ischemia. It helps do so by speeding up functional recovery through supplying more-than usual blood to nourish the deprived heart muscle and wash away accumulated metabolic byproducts (105). Therefore, CRH, as is reactive hyperemia elsewhere in the body, may be viewed as a protective mechanism against ischemia. Research has shown that $\mathrm{CRH}$ is attenuated in pathologic conditions affecting blood vessels including the coronary blood vessels. Previous research has also confirmed that $\mathrm{CRH}$ is decreased in cardiac hypertrophy (36) and metabolic syndrome (6). Moreover, higher risk of cardiovascular conditions, such as unstable angina, myocardial infarction, congestive heart failure and cardiac death, are linked to decreased CRH (30). Additionally, corrective measures have been shown to reverse or mitigate this association. Patients with acute myocardial infarction, who had blunted CRH, had significant improvement in CRH after successful coronary angioplasty as measured by coronary angiography (91). Another useful application of CRH is that it serves as a measure of coronary vascular reserve (CVR), which is traditionally measured 
using pharmacological agents. However, $\mathrm{CRH}$ is an accepted alternative for estimating CVR (38). A number of parameters can be evaluated during $\mathrm{CRH}$; figure I illustrates these parameters. Additionally, heart rate (HR) and left ventricular developed pressure (LVDP) are used to evaluate the basic function of the heart in response to $\mathrm{CRH}$ and the drugs used to study it. Extensive work has been done to study the mechanisms and mediators involved in reactive hyperemia. These mechanisms may be broadly divided into two categories, mechano-senstive and metabolic (105). The mechano-senstive mechanisms include shear stress and myogenic response. On the other hand, the list of metabolic mediators include adenosine, which plays an important role through acting on its receptor subtypes $\mathrm{A}_{2 \mathrm{~A}}$ and $\mathrm{A}_{2 \mathrm{~B}}(5,36)$, nitric oxide (NO) (36), ATP-dependent $\mathrm{K}^{+}\left(\mathrm{K}_{\mathrm{ATP}}\right)$ channels, which function downstream of adenosine $(36,80)$, and hydrogen peroxide $\left(\mathrm{H}_{2} \mathrm{O}_{2}\right)$, which functions downstream of adenosine $(36,80) . \mathrm{H}_{2} \mathrm{O}_{2}$ is one of the endothelium-derived hyperpolarizing factors (EDHFs). Likewise, Campbell et al. identified epoxyeicosatrienoic acids (EETs) as EDHF (9). 


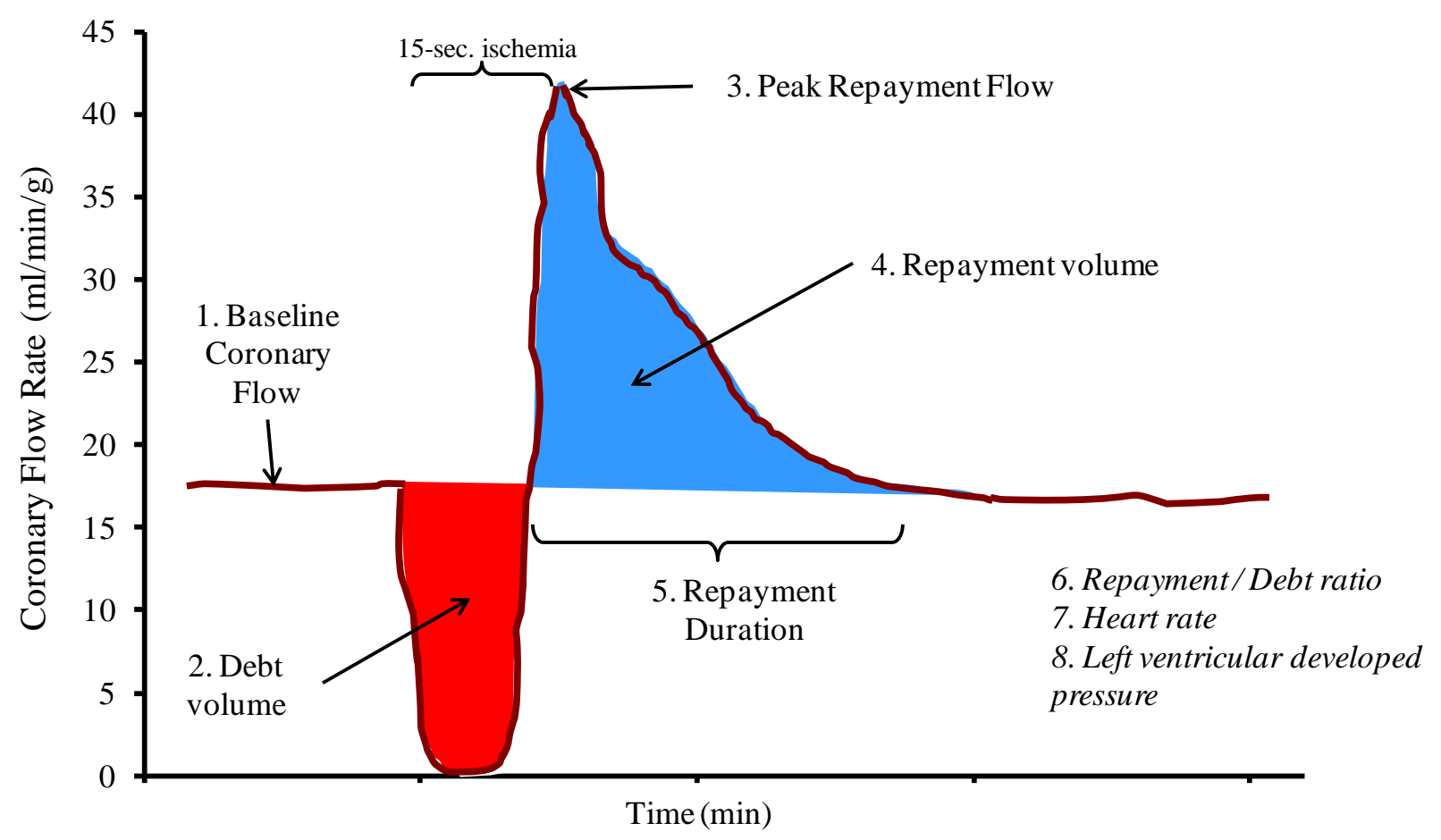

Figure I: Different parameters derived from coronary reactive hyperemia before and after 15-sec no-flow ischemia 


\section{Oxylipins}

Oxylipins are bioactive lipids generated by the oxidation of polyunsaturated fatty acids (PUFAs) (95). Since their discovery almost five decades ago, numerous biologic functions have been linked to them, and many others are still being elucidated. The advancement in detection and quantification rejuvenated both interest and research in oxylipins with accurate nanomolar detection using an array state-of-the-art mass spectrometry instruments (89). Still, studying the biologic functions of oxylipins is challenged by the sheer number of oxylipins discovered thus far, over 100 oxylipins have been discovered (95), and the overlapping and interconnected roles different oxylipin groups have. Important cardiovascular disease (CVD) pathologies, including hyperlipidemia, hypertension, thrombosis, hemostasis, and diabetes have been linked to abnormal oxylipin signaling (25).

Oxylipins derived from arachidonic acid (AA) include EETs, HETEs, and prostanoids, whereas those derived from linoleic acid (LA) include EpOMEs and HODEs among others. Figure II illustrates some of AA- and LA-derived oxylipins and the main enzymes involved in their generation and breakdown, such as CYP epoxygenases and sEH.

\section{Biosynthesis:}

Oxylipins are both potent and short lived. Therefore, they are not stored; they are rather synthesized de novo and regulated closely, and exert their effect in a paracrine or autocrine manner (95). Free PUFAs are mono- or dioxygenated by three families of enzymes: cyclooxygenase (COX), lipoxygenase (LOX), and cytochrome P450 (CYP) into distinct classes of oxylipins (50). The type of oxylipins produced from PUFAs depends on the amount of dietary PUFAs consumed, the oxygenases (COX, LOX or CYP) present for metabolizing PUFAs, and 
the enzyme's affinity for a specific substrate PUFA (22). The most well-known oxylipins are the eicosanoids (20-carbon compounds) formed from arachidonic acid (AA), and octadecanoids (18carbon compounds) derived from linoleic acid (LA) (22). Cyclooxygenase (COX) enzymes convert AA into prostanoids (PGs and thromboxanes). Also, COX enzymes can produce some hydroxy-metabolites, such as 11-HETE from AA, and 9-HODE from LA (94). Lipoxygenases (LOXs) catalyze the formation of hydroxy fatty acids, including leukotrienes, lipoxins, resolvins, protectins, maresins, hepoxilins, and eoxins (22). LOX enzymes also metabolize AA to form mid-chain (5-, 8-, 9-, 11-, 12-, and 15-) HETEs (3). Cytochrome P450 (CYP) enzymes, which were originally known for their roles in xenobiotic metabolism, could either have epoxygenase or $\omega$-hydroxylase activity (22). CYP $\omega$-hydroxylase enzymes (CYP4A and CYP4F) metabolize AA and generate $\omega$-terminal (16-, 17-, 18-, 19-, and 20-) HETEs, whereas CYPs with epoxygenase activity (CYP2C and CYP2J) metabolize AA and generate epoxyeicosatrienoic acid (EETs), which are further metabolized to dihydroxyeicosatrienoic acids (DHETs) by soluble epoxide hydrolase ( $\mathrm{sEH}$ ) (95). AA can also be generated from LA metabolism (40). Metabolism of LA includes the same enzyme families described above; for example, CYP epoxygenases metabolize LA to form EpOMEs, the epoxy compounds of LA. EpOMEs are hydrated by sEH to form DiHOMEs, the dihydroxy form of EpOMEs (3). LOX enzymes form the hydroxy metabolites of LA: HODEs (95). Oxylipins have a wide range of biological functions, many of which are still being investigated. They produce their effects through activating PPARs, or through GPCRs (81). Targeting sEH impacted the level of oxylipins directly affected by its catalytic activity, such as EETs, DHETs, EpOMEs, and DiHOMEs, and indirectly by affecting the other PUFAs pathways, such as HODEs, and HETEs $(45,47)$. The latter observation could 
be explained by the shift observed in $\mathrm{sEH}^{-1-}$ in AA metabolism due to EETs accumulation (39), suggesting that the different oxylipin pathways affect one another.

\section{Epoxyeicosatrienoic acids (EETs)}

EETs are 20-carbon metabolites of arachidonic acid (AA) with numerous physiologic actions. They are generated from AA by the cytochrome P450 epoxygenase pathway. Four distinct regioisomers are produced: 5,6-EET, 8,9-EET, 11,12-EET, and 14,15-EET. In the heart, EETs exert cardioprotective effects in ischemia / reperfusion injury (77). EETs are classified as EDHFs; they are produced in endothelial cells and induce hyperpolarization in vascular smooth muscle cells by activating large conductance $\mathrm{Ca}^{2+}$-activated $\mathrm{K}^{+}$channels $\left(\mathrm{BK}_{\mathrm{Ca}}\right)(9,20)$. EETs also cause vasodilation in many vascular beds such as the intestines (71), kidney preglomerular vasculature (33), conduit arteries (57-61), and brain (23). It is worth mentioning that small $\left(\mathrm{K}_{\mathrm{Ca}} 2.3\right)$ and medium $\left(\mathrm{K}_{\mathrm{Ca}} 3.1\right)$ conductance $\mathrm{Ca}^{2+}$-activated $\mathrm{K}^{+}$channels are important in EETsinduced hyperpolarization (20). EETs are metabolized rapidly by hydration to their corresponding, less active, dihydroxyeicosatrienoic acids (DHETs) by soluble epoxide hydrolase sEH, which is the main catabolic pathway responsible for EETs breakdown (83). Not all EETs isomers are substrates for sEH; 5,6-EET is a poor substrate of sEH. In fact, this EET isomer along with 8,9-EET are substrates for cyclooxygenase (COX) pathway (32). The terminal halflife of 14,15-EET was found to be between 7.9 - 12.3 minutes (10). Other catabolic pathways of EETs include $\omega$-oxidation, $\beta$-oxidation, and chain elongation. The latter two pathways become more important when the activity of the main pathway, hydration by $\mathrm{sEH}$, is inhibited (32). Not

only were EETs shown to have confirmed beneficial effects in numerous animal studies through their vasodilatory $(23,33,57-61,71)$, cardioprotective (77), and anti-inflammatory effects (79), 
they were also linked to decreased cardiovascular risk in epidemiological studies in humans (42, 43). Polymorphism variants where EETs production is decreased, such as decreased CYP2J2 expression (variant G-50T) (42) or EETs breakdown is increased, such as increased sEH activity (variant K55R) (43), had increased risk of coronary artery disease. It was speculated by some researchers that EETs exert their effects through specific cell surface receptors, which is supported by the finding that different responses were elicited by different stereoisomers and regioisomers of EETs (100). However, it is worth mentioning that numerous reports linked EETs's signaling pathway with protein kinase A (PKA) and cAMP (63).

\section{Mid-chain Hydroxyeicosatetraenoic Acids (HETEs):}

Mid-chain HETEs are produced through allylic oxidation of AA by lipoxygenase (LOX) (39). They were shown to have chemotaxis effects, change vascular tone, and induce the production of vascular endothelial growth factors $(29,56,86,93)$. Also, the increased formation of mid-chain HETEs was involved in cardiovascular dysfunction $(13,68,87,97)$. Unlike the vasodilatory effect of EETs in the kidneys (33), 12-HETE caused vasoconstriction in small renal arteries (48). Also, the generation of mid-chain HETEs is increased in essential hypertension (16) suggesting that they could be involved in its pathogenesis. These reports point to opposite effects of EETs and HETEs in vascular biology. Maayah et al. reported that mid-chain HETEs blocked the synthesis of EETs and increased their conversion to DHETs in RL-14 cells (49). Moreover, although sEH is not directly involved in the generation or breakdown of mid-chain HETEs, sEH was found to be essential for mid-chain HETE-mediated induction of cellular hypertrophy (49). Therefore, not only do EETs and mid-chain HETEs have opposite effects, they seem to affect the level of each other. 


\section{Epoxyoctadecaenoic acid (EpOMEs):}

The physiological significance of EpOMEs and DiHOMEs remains poorly understood, and the evidence is somewhat contradictory, with a few studies suggesting toxic effects, while others indicating beneficial effects of these bioactive metabolites. EpOMEs and DiHOMEs were reported to increase oxidative stress in vascular endothelial cells (96); DiHOMEs were toxic to renal proximal tubular cells (54); and intravenously injected 9,10-EpOME had cardiodepressive effects in dogs (90). The main caveat of these studies, which reported that EpOMEs and DiHOMEs have toxic effects, is that very high concentrations $(100-500 \mu \mathrm{M})$ were used (39). Contrary to these reports, lower, more physiological concentrations of EpOMEs and DiHOMEs did not have toxic effects and rather had beneficial effects (52). Mitchell et al reported that LA and its oxidative metabolites (EpOMEs and DiHOMEs) did not have toxic effects during acute exposure in Langendorff-perfused rat hearts (52). Pretreatment with 12,13-EpOME protected primary cultures of rabbit renal proximal tubular cells against hypoxia/reoxygenation injury (65). Also, increased EpOME/DiHOME ratio, induced by sEH inhibition using AUDA, improved renal recovery in response to ischemia/reperfusion injury in C57BL/6 mice (44).

\section{Hydroxyoctadecadienoic Acids (HODEs):}

Linoleic acid (LA) is also metabolized through hydroxylation by CYP epoxygenases to form hydroxyl-LA metabolites known as hydroxyoctadecadienoic acids (HODEs) (39). Like EpOMEs, the physiologic functions of HODEs are still being investigated (39). 13-HODE is suggested to have an anti-inflammatory role in inflammatory diseases through its effect as a PPAR $\gamma$-agonist $(2,4,18,21,88)$. Also, 13-HODE, through increasing prostacyclin $\left(\mathrm{PGI}_{2}\right)$ biosynthesis, was involved in splenic and coronary artery relaxation in smooth muscle cells in Mongrel dogs (15). 
9-HODE, unlike 13-HODE, was described as pro-inflammatory in an experimental woundhealing model in rats $(26,66)$.

\section{Prostanoids:}

The term prostanoids comprises two distinct groups: prostaglandins and thromboxanes. Prostaglandins $\mathrm{G}_{2}$ and $\mathrm{H}_{2}$, which are AA metabolites formed by COX isoforms (1 and 2), are converted to the 4 main bioactive PGs $\left(\mathrm{D}_{2}, \mathrm{E}_{2}, \mathrm{I}_{2}\right.$, and $\left.\mathrm{F}_{2 \alpha}\right)$ and thromboxanes $\left(\mathrm{TxA}_{2}\right.$ and $\left.\mathrm{TxB}_{2}\right)$ $(74,92)$. Most PGs have pro-inflammatory effects. For example, PG-E $E_{2}$ augments arterial dilation and increases microvascular permeability (92). However, PG-E $E_{2}$ was found to have an antiinflammatory role as well by up-regulating cAMP and inducing secretion of the antiinflammatory IL-10 (78). Similarly, PG-D 2 attenuated inflammation in experimental models of pleuritis and colitis (92). 6-keto-PG- $\mathrm{F}_{1 \alpha}$, the non-active hydrolysis product of prostacyclin (PG$\mathrm{I}_{2}$ ), and $\mathrm{TxB}_{2}$, the inactive degradation product of $\mathrm{TxA}_{2}(92)$, were also decreased by $t$-AUCB. Targeting sEH was shown to affect the level of different PGs. For example, sEH-inhibition by AUDA-BE reversed lipopolysaccharide (LPS)-induced increase in PG-D 2, PG-E 2 , 6-keto-PG-F ${ }_{1 \alpha}$, and $\mathrm{TxB}_{2}$ (76). Also, sEH-inhibition by AUDA-BE, through increasing the concentrations of EETs, inhibited NF- $\mathrm{B}$ translocation (75). Combination of sEH-inhibition by AUDA-BE and COX-inhibition had an additive effect and further decreased PG- $\mathrm{D}_{2}$ and $\mathrm{PG}-\mathrm{E}_{2}(75)$. 


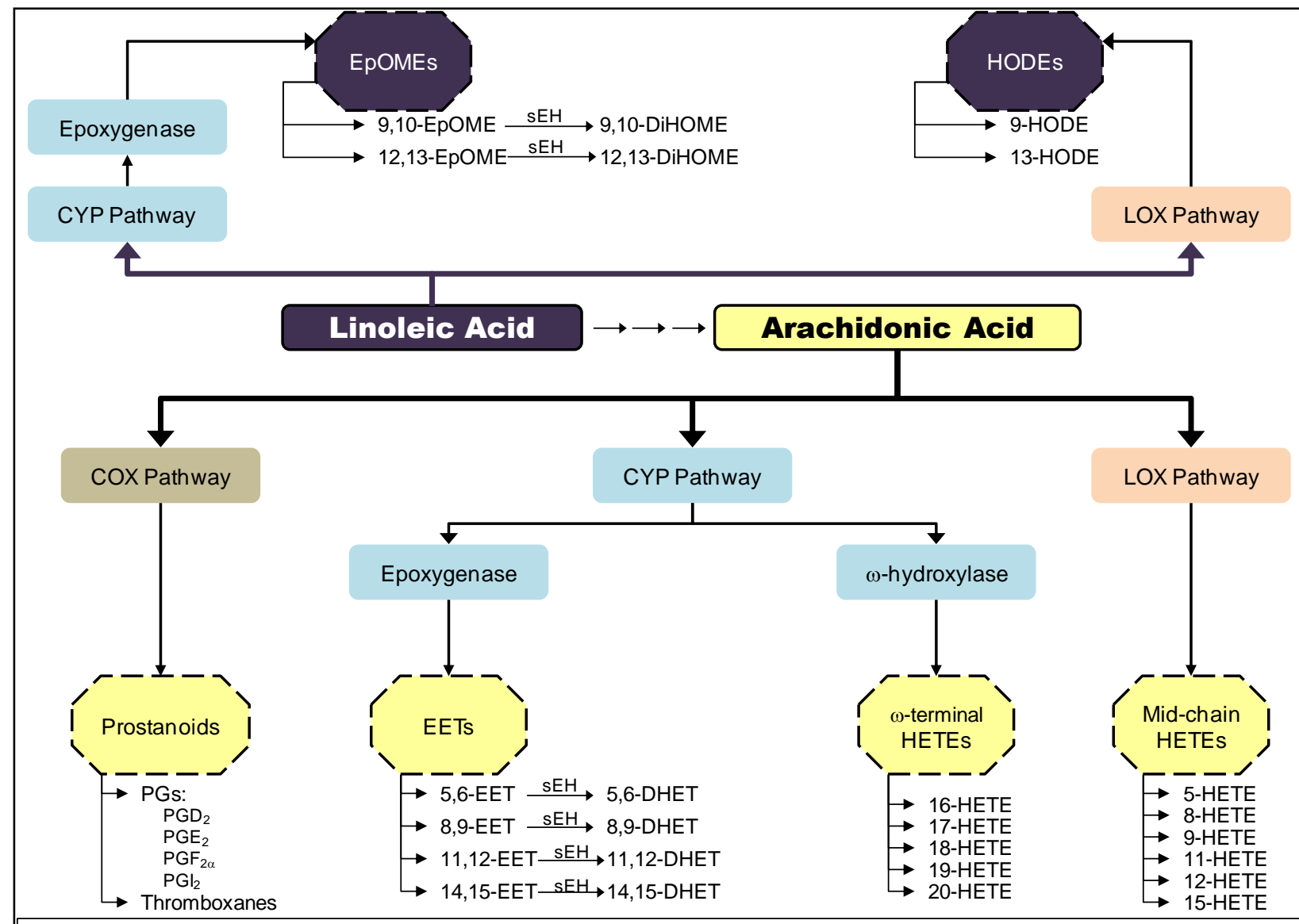

Figure II: Oxylipins derived from arachidonic and linoleic acids and the pathways involved in their generation and catabolism. (Tam et al., 2013) 


\section{Coronary Reactive Hyperemia in the Isolated Perfused Heart}

The Langendorff perfused heart technique is well established. It has evolved substantially since it was first conceived for mammalian hearts by Oscar Langendorff in 1897 (84). Of the two available types of this technique, we chose the constant pressure mode to prefuse the heart over the constant flow mode. In the latter mode, the amount of perfusate is not physiologically modulated in response to the changing needs of the heart muscle as a result of overriding the autoregulatory mechanisms by providing constant flow of the perfusate. This is especially important when trying to assess the role of coronary regulatory mechanisms in response to changing coronary flow conditions or pathologies such as ischemia. Based on that, the constant pressure mode is physiologically more relevant in experiments involving ischemia (84). Figure III illustrates a schematic picture showing the setup of Langendorff perfused heart experiment. Although the Langendorff perfused heart technique has been criticized for a number of limitations, its overall advantages and reproducibility still make it a valuable tool for studying both physiologic and pathologic changes in the heart, particularly when cardiac ischemia or hypoxia are being investigated. Some of the reported disadvantages include a requirement of certain level of skill in handling the heart and preparing it for experiments. This is particularly noticed when dealing with small animals such as mice; it is rather a demanding technique and takes some time to develop the skill for it. Another related point, which is critical for the integrity of the heart, is the time the heart spends under ischemic state; it is absolutely vital to instantly place the excised heart in ice-cold buffer to slow the metabolism and therefore diminish the oxygen requirements of the heart. Removing the surrounding tissues, such as lungs, trachea and blood vessels, and preparing the aorta for hanging should be quick and done while the heart is immersed in the ice-cold buffer. Once hanged and fixed in place, the already warmed and 
oxygenated buffer should be allowed to perfuse the heart. Besides taking all the above mentioned precautions into consideration, we perform an extra final step to make sure that the heart endothelium is still intact and the heart is still suitable for experimentation. This step is the induction of reactive hyperemia in response to a $15 \mathrm{sec}$ ischemia, which happens to be the main variable in our study. This has been the golden standard to ascertain the integrity of the heart. We only proceed with our experiment if the maximum coronary flow in response to the $15 \mathrm{sec}$ ischemia is increased by at least 2 or more fold.

Also, in Langendorff technique, the fact that the heart is disconnected from the body and the extrinsic control of the humoral and nervous systems could be viewed as a disadvantage. Actually, this may be considered an advantage as it allows the evaluation of drug effects on the heart while excluding any extrinsic effect, which could modulate its response. Another reason for using the Langendorff perfused heart technique is that the epoxygenase pathway is not functional in cell culture systems (32). 


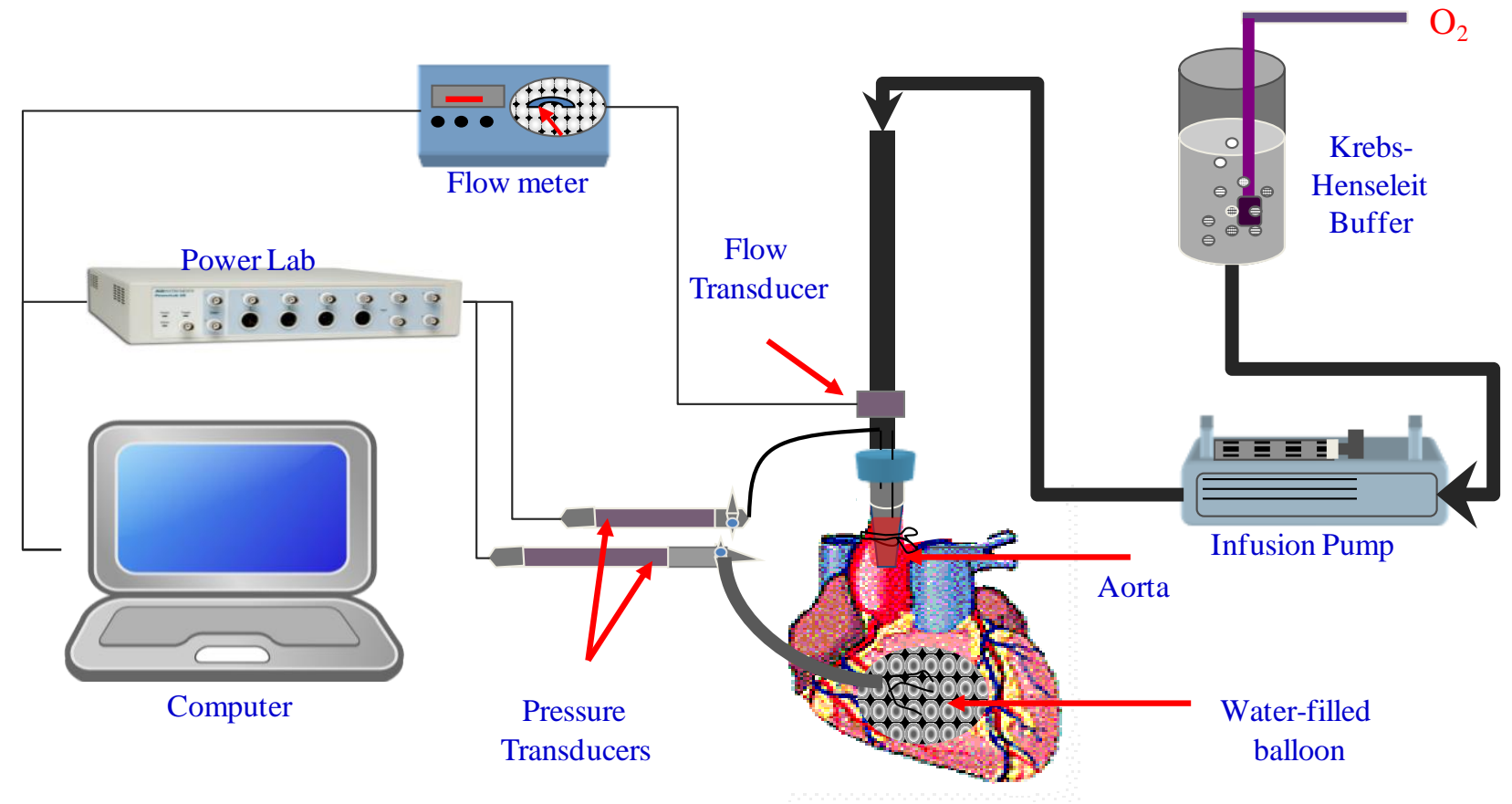

Figure III: Schematic picture showing the setup of Langendorff perfused heart experiment 


\section{Peroxisome Proliferator-Activated Receptors (PPARs)}

PPARs are nuclear receptors, which, upon activation, modulate DNA transcription of target genes (98). Three subtypes of PPARs are identified: $\alpha, \beta / \delta$, and $\gamma(51)$. Because of their large ligand-binding domain, PPARs can bind to a large number of ligands $(64,99)$. PPARs are involved in many biologic functions, such as vascular tone regulation, inflammation, energy homeostasis, adipogenesis, and insulin sensitivity $(11,31,64,99)$. Many of EETs' effects have been linked to $\operatorname{PPAR} \gamma(59,70)$. The anti-inflammatory effect of EETs in cultured endothelial cells was induced by laminar flow and mediated by PPAR $\gamma$ (46). Cowart et al reported that EETs metabolites formed by Cyp4a were high-affinity ligands of PPAR $\gamma$ (14). Also, Cai et al reported that EETs protected against Angiotensin II - induced abdominal aortic aneurysm in cyp2j2overexpressed mice through $\operatorname{PPAR} \gamma(8)$. These $(8,14,46,59,70)$ and other reports prompted us to investigate a possible link among CYP-epoxygenases, sEH, oxylipins and PPAR $\gamma$ in modulating CRH response. 


\section{Hypothesis and Project Summary:}

Coronary reactive hyperemia $(\mathrm{CRH})$ is a physiological response to ischemic insult that prevents the potential harm associated with ischemia or interruption of blood supply. The relationship between the pharmacologic inhibition or genetic deletion of soluble epoxide hydrolase (sEH) and CRH response to a brief ischemia is not known. EETs protect against ischemia/reperfusion injury and have numerous beneficial physiological effects. sEH is involved in the main catabolic pathway of epoxyeicosatrienoic acids (EETs), which are converted into dihydroxyeicosatrienoic acids (DHETs). Oxylipins are inter-connected through sharing common precursor molecules and through affecting each others' levels. The potential role of targeting sEH in coronary flow (CF) during CRH in response to short ischemia has not been investigated. Therefore, we hypothesized that targeting $\mathrm{sEH}$, through pharmacologic inhibition or genetic deletion, enhances $\mathrm{CRH}$ in isolated mouse hearts through changing the oxylipin profiles.

To test this hypothesis, we had the following specific aims:

\section{- Specific Aim 1:}

Investigate the effect of sEH deletion on CRH and oxylipins using gene manipulated sEH-knockout $\left(\mathrm{sEH}^{--}\right)$and wild type $\left(\mathrm{sEH}^{+/+}\right)$mice.

\section{- Specific Aim 2:}

Investigate the effect of sEH inhibition on $\mathrm{CRH}$ and oxylipins using pharmacology in wild type $\left(\mathrm{sEH}^{+/+}\right)$mice.

\section{- Specific Aim 3:}

Explore the role of PPAR $\gamma$ in the downstream signaling pathway involved in $\mathrm{CRH}$ through disruption of sEH using gene manipulated technology and pharmacology. 


\title{
CHAPTER ONE
}

\section{Deletion of Soluble Epoxide Hydrolase Enhances Coronary Reactive Hyperemia in Isolated Mouse Heart: Role of Oxylipins and PPAR $\gamma$ \\ (This chapter was published in the American Journal of Physiology-Regulatory, Integrative and Comparative Physiology) \\ (2016 Oct 1;311(4):R676-R688. doi: 10.1152/ajpregu.00237.2016. Epub 2016 Aug 3.)}

\author{
Ahmad Hanif ${ }^{1}$, Matthew L. Edin ${ }^{2}$, Darryl C. Zeldin ${ }^{2}$, Christophe Morisseau ${ }^{3}$, and Mohammed A. \\ Nayeem $^{1}$ \\ ${ }^{1}$ Basic Pharmaceutical Sciences, School of Pharmacy, Center for Basic and Translational Stroke \\ Research. West Virginia University, Morgantown, WV \\ ${ }^{2}$ Division of Intramural Research, NIEHS/NIH, Research Triangle Park, NC \\ ${ }^{3}$ University of California at Davis, One Shields Avenue, Davis, CA
}

\begin{abstract}
:
The relationship between soluble epoxide hydrolase $(\mathrm{sEH})$ and coronary reactive hyperemia (CRH) response to a brief ischemic insult is not known. Epoxyeicosatrienoic acids (EETs) exert cardioprotective effects in ischemia/reperfusion injury. sEH converts EETs into dihydroxyeicosatrienoic-acids (DHETs). Therefore, we hypothesized that knocking out sEH enhances CRH through modulation of oxylipin profiles including an increase in EETs/DHETs ratio. Compared to $\mathrm{sEH}^{+/+}, \mathrm{sEH}^{-/}$mice showed enhanced $\mathrm{CRH}$, including greater repayment volume (RV; $28 \%$ higher, $\mathrm{p}<0.001)$ and repayment/debt ratio (32\% higher, $\mathrm{p}<0.001)$. Oxylipins
\end{abstract}


from the heart perfusates were analyzed by LC-MS/MS. The 14,15-EET/14,15-DHET ratio was 3.7 fold higher at baseline $(\mathrm{p}<0.001)$ and 5.6 fold higher post-ischemia $(\mathrm{p}<0.001)$ in $\mathrm{sEH}^{-/}$ compared to $\mathrm{SEH}^{+/+}$mice. Likewise, the baseline 9,10- and 12,13-EpOME/DiHOME ratios were $3.2(\mathrm{p}<0.01)$ and $3.7(\mathrm{p}<0.001)$ fold higher, respectively in $\mathrm{sEH}^{-/-}$compared to $\mathrm{SEH}^{+/+}$mice. 13HODE was also significantly increased at baseline by $71 \%(\mathrm{p}<0.01)$ in $\mathrm{sEH}^{-/-}$vs. $\mathrm{sEH}^{+/+}$mice. Levels of 5-, 11-, 12-, and 15-HETEs were not significantly different between the two strains $(\mathrm{p}>0.05)$, but were decreased post-ischemia in both groups $(\mathrm{p}=0.02, \mathrm{p}=0.04, \mathrm{p}=0.05, \mathrm{p}=0.03$ respectively). Modulation of $\mathrm{CRH}$ by $\operatorname{PPAR} \gamma$ was demonstrated using a PPAR $\gamma$-antagonist (T0070907), which reduced repayment volume by $25 \%$ in $\mathrm{sEH}^{+/+}(\mathrm{p}<0.001)$ and $33 \%$ in $\mathrm{sEH}^{-/-}$ mice ( $\mathrm{p}<0.01$ ), and a PPAR $\gamma$-agonist (rosiglitazone), which increased repayment volume by $37 \%$ in both $\mathrm{sEH}^{+/+}(\mathrm{p}=0.04)$ and $\mathrm{sEH}^{-/-}$mice $(\mathrm{p}=0.04)$. L-NAME attenuated $\mathrm{CRH}$ in both $\mathrm{sEH}^{-/-}$and $\mathrm{sEH}^{+/+}$. These data demonstrate that genetic deletion of $\mathrm{sEH}$ resulted in an altered oxylipin profile which may have led to an enhanced CRH response.

\section{Introduction}

During reactive hyperemia $(\mathrm{RH})$, blood flow increases transiently in response to ischemia or interruption of blood supply (105). In the heart, $\mathrm{RH}$ is referred to as coronary RH (CRH), and is associated with coronary vasodilation in response to cessation in coronary perfusion. This occlusion or ischemic insult is inherently associated with potentially detrimental effects on the heart and cardiac function. The increase in blood flow associated with CRH increases repayment volume (RV) to prevent or decrease potential injury due to ischemia. CRH speeds up functional recovery by supplying more than the usual amount of blood to nourish the deprived heart muscle, 
and wash away accumulated metabolic byproducts (105); therefore, CRH may be viewed as a protective mechanism. Research has shown that RH is blunted in pathologic conditions affecting blood flow including the coronary circulation; CRH is decreased in cardiac hypertrophy (36) and metabolic syndrome (6). Moreover, higher risk for cardiovascular diseases, such as unstable angina, myocardial infarction, congestive heart failure, and cardiac death are linked to decreased CRH (30). Corrective measures, such as coronary angioplasty, have been shown to reverse or mitigate decreased $\mathrm{CRH}$ in patients with acute myocardial infarction, and significantly improved CRH after successful coronary angioplasty (91). Although coronary vascular reserve is traditionally measured through pharmacological agents, $\mathrm{CRH}$ is also an accepted alternative to measure it if patients cannot tolerate the pharmacological agents (38).

The mechanisms and mediators involved in $\mathrm{RH}$ have been studied extensively. These mechanisms may be broadly divided into two categories: mechanosensitive and metabolic (105). The mechanosensitive mechanisms include shear stress and myogenic response. Metabolic mediators involved in $\mathrm{RH}$ include adenosine $(5,36)$, nitric oxide (NO) (36), $\mathrm{K}_{\mathrm{ATP}}$ channels (36) and hydrogen peroxide $\left(\mathrm{H}_{2} \mathrm{O}_{2}\right)(36,80)$.

Epoxyeicosatrienoic acids (EETs) are oxidized metabolites of arachidonic acid (AA) that have numerous physiologic actions. EETs are produced in endothelial cells and induce hyperpolarization and relaxation in vascular smooth muscle cells by activating large conductance $\mathrm{Ca}^{2+}$-activated $\mathrm{K}^{+}$channels $\left(\mathrm{BK}_{\mathrm{Ca}}\right)(9,20)$. EETs are rapidly metabolized by soluble epoxide hydrolase $(\mathrm{sEH})$ to less active dihydroxyeicosatrienoic acids (DHETs) through hydration, the main catabolic pathway responsible for EETs breakdown (83); therefore, targeting sEH is associated with beneficial effects on the cardiovascular system $(19,59)$. Also, others $(17,39,45)$ have reported that $\mathrm{sEH}^{-/-}$mice were associated with elevated EETs levels compared to $\mathrm{sEH}^{+/+}$ 
mice, and EETs exerted cardioprotective effects against ischemia/reperfusion injury (77). Additionally, targeting sEH impacted the level of oxylipins, such as EpOMEs, DiHOMEs, HODEs, and HETEs (45, 47). Lee et al., (44) reported that EpOMEs/DiHOMEs ratio was increased in response to the sEH inhibitor 12-(3-adamantan-1-yl-ureido)-dodecanoic acid (AUDA) in vivo; this change was associated with improved renal recovery against ischemia reperfusion injury in C57BL/6 mice. Guido et al., (15) reported that 13-HODE (hydroxyoctadecadienoic acids) was involved in canine splenic and coronary artery relaxation through $\mathrm{PGI}_{2}$ (prostacyclin) biosynthesis in both endothelial and smooth muscle cells. Mid-chain HETEs (hydroxyeicosatetraenoic acids) had direct chemo-toxic effects on vascular tone and production of endothelial vascular factors, such as vascular endothelial growth factor (VEGF), and pigment epithelium-derived factor $(\mathrm{PEDF})(1,29,56,86,93)$. Further, EETs-induced aortic relaxation in mice was mediated by peroxisome proliferator-activated receptor gamma (PPAR $\gamma$ ) $(59,70)$. Also, in mouse mesenteric arteries, EETs-induced vasodilatory effects were inhibited by L-NAME (a non-selective NO synthase, NOS, inhibitor) (28). Seubert et al., (77) reported that CYP2J2 over-expression in the mouse heart involved increased generation of EETs compared to their respective control and exerted cardioprotective effects against ischemia/reperfusion injury. However, the potential role of sEH deletion in coronary flow (CF) during CRH in response to short ischemia has not been investigated. Therefore, we hypothesized that knocking out sEH enhances $\mathrm{CRH}$ in isolated mouse heart through modulation of oxylipin profiles including an increase in EETs/DHETs ratio.

\section{Materials and Methods}

\section{Animals}


The generation of $\mathrm{sEH}^{-/-}$mice was described by Sinal et al. (83). $\mathrm{sEH}^{-/-}$and $\mathrm{sEH}^{+/+}$mice were provided by Dr. Darryl Zeldin, National Institute of Environmental Health Sciences / National Institutes of Health (NIH). All animal care and experimentation protocols were approved and carried out in accordance with the West Virginia University Institutional Animal Care and Use Committee and were in accordance with the principles and guidelines of the NIH's Guide for the Care and Use of Laboratory Animals. Both male and female mice (14-16 wks old) in equal ratio were used in our study. Mice were maintained in cages with a 12:12 h light-dark cycle and free access to standard chow and water.

\section{Langendorff-Perfused Heart Preparation}

The Langendorff perfused heart preparation is a well-established technique for studying the cardiac functions and pathologies in vitro. There are two available Langendorff technique modes to perfuse the heart: 1) constant pressure mode, and 2) constant flow mode. In our experiments, we chose the constant pressure mode. The selection between the two is especially important when trying to assess the role of coronary regulatory mechanisms in response to changing $\mathrm{CF}$ conditions or pathologies such as ischemia. Based on that, the constant pressure mode is physiologically more relevant in experiments involving ischemia, like the model in our experiments (84).

Deletion of $\mathrm{sEH}$ in $\mathrm{sEH}^{-/-}$mice was confirmed by genotyping of mouse tail samples (data not shown). Also, Western blotting confirmed the deletion of $\mathrm{sEH}$ protein in $\mathrm{sEH}^{-/}$mice aortic samples and was reported by our laboratory (59). Soluble epoxide hydrolase null $\left(\mathrm{sEH}^{--}\right)$and wild-type mice $\left(\mathrm{sEH}^{++}\right)$mice $(14-16$ wks.) of both sexes (equal ratios) were euthanized with 
sodium pentobarbital $(100 \mathrm{mg} / \mathrm{kg}$ body weight intra-peritoneally). Hearts were excised and immediately placed into heparinized $(5 \mathrm{U} / \mathrm{mL})$ ice-cold Krebs-Henseleit buffer containing (in mM) $119.0 \mathrm{NaCl}, 11.0$ glucose, $22.0 \mathrm{NaHCO}_{3}, 4.7 \mathrm{KCl}, 1.2 \mathrm{KH}_{2} \mathrm{PO}_{4}, 1.2 \mathrm{MgSO}_{4}, 2.5 \mathrm{CaCl}_{2}, 2.0$ pyruvate, and 0.5 EDTA. After removal of the lung and surrounding tissue around the heart, the aorta was rapidly cannulated with a 20 -gauge, blunt-ended needle and continuously perfused with $37^{\circ} \mathrm{C}$ buffer continuously bubbled with $\left(\left[95 \% \mathrm{O}_{2}\right]-\left[5 \% \mathrm{CO}_{2}\right]\right)$ at a constant perfusion pressure of $80 \mathrm{mmHg}$. The left atrium was excised, and a water-filled balloon made of plastic wrap was inserted into the left ventricle through the mitral valve. The balloon was connected to a pressure transducer for continuous measurement of left ventricular developed pressure (LVDP) and heart rate (HR). The heart was then immersed in a water-jacketed perfusate bath $\left(37^{\circ} \mathrm{C}\right)$ and left to beat spontaneously. Left ventricular diastolic pressure was adjusted to $2-5 \mathrm{mmHg}$. A flow transducer was installed above the cannulated aorta for continuous measurement of CF with an ultrasonic flow probe (Transonic Systems, Ithaca, NY). A Power-Lab Chart data acquisition system (AD Instruments, Colorado Springs, CO) was used for data acquisition. Heart was allowed to stabilize for 30-40 min before initiation of CRH. Only hearts whose peak CF increased by more than two fold after a 15 -second total occlusion were included in the analysis. This demonstrated that the isolated heart preparation was intact to be included in the experiment. Hearts with persistent arrhythmias or LVDP of less than $80 \mathrm{mmHg}$ were excluded. 9 mice (4 males and 5 females), out of 58 mice, were excluded from our study for the mentioned reasons.

\section{Coronary Reactive Hyperemic Response:}

After stabilization for 30-40 minutes, baseline CF, HR, and LVDP were recorded. Hearts were subjected to 15 seconds of total occlusion by closing the valve directly above the cannulated 
heart to bring forth $\mathrm{CRH}$. After $\mathrm{CF}$ returned to pre-CRH baseline levels, post-CRH baseline $\mathrm{CF}$, CF tracing, peak hyperemic flow (PHF), HR, LVDP, repayment volume (RV), and repayment duration $(\mathrm{RD})$ recordings were analyzed for each isolated heart. Investigation drugs were infused into the aortic perfusion line using a microinjection pump (Harvard Apparatus, Holliston, MA) for 15 minutes, after which another $\mathrm{CRH}$ was induced and the same parameters analyzed again. Drugs were infused at a rate equivalent to $1 \%$ of $\mathrm{CF}$. The final concentrations, after standardization of dose $(0.01,0.1,1, \& 10 \mu \mathrm{M})$ response for the various drugs used in this study were $10 \mu \mathrm{M}$ for T0070907 (PPAR $\gamma$-antagonist), rosiglitazone (PPAR $\gamma$-agonist), and $100 \mu \mathrm{M}$ for

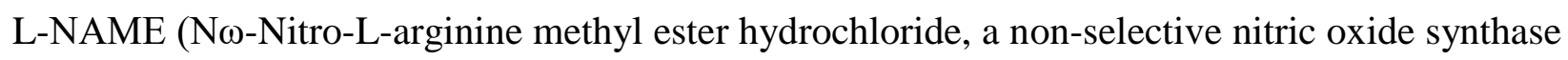
inhibitor). These concentrations were selected based on our dose-response studies (for T0070907 and rosiglitazone) and based on the concentrations used in previous studies (rosiglitazone, 10 $\mu \mathrm{M}$; (67)), and (L-NAME, $100 \mu \mathrm{M}$; (105)). Time-matched control experiments with WT $\left(\mathrm{sEH}^{+/+}\right)$mouse hearts, employing three consecutive inductions of $\mathrm{CRH}$, showed no change in CRH response and in baseline heart functions, including CF, LVDP, and HR (data not shown).

\section{LC-MS/MS Oxylipin Analysis}

Oxylipin levels (5,6-, 8,9-, 11,12- and 14,15-EETs; 5,6-, 8,9-, 11,12- and 14,15-DHETs; 5-, 8-, 9-, 11-, 12- and 15-HETEs; 9,10- and 12,13-EpOMEs; 9,10- and 12,13-DiHOME; 9- and 13HODEs) were analyzed in pre- and post CRH heart perfusates of $\mathrm{sEH}^{-/-}$and $\mathrm{sEH}^{+/+}$mice through liquid chromatography, tandem mass spectroscopy (LC-MS/MS) as described previously (41). Briefly, heart perfusates were collected after the first 30 min of stabilization, and right after reperfusion for $2.5 \mathrm{~min}$. Hearts were immersed in $5 \mathrm{~mL}$ of warm $\left(37^{\circ} \mathrm{C}\right) \mathrm{Krebs}-$ Henseleit buffer and $5 \mu \mathrm{L}$ of $10 \mu \mathrm{M} t$-AUCB (trans-4-[4-(3-adamantan-1-yl-ureido)- 
cyclohexyloxy]-benzoic acid, selective sEH-inhibitor) to block further breakdown of EETs and EpOMEs by sEH. Heart perfusates were collected two times before (at baseline) and pooled together as one sample, and two times after ischemia and pooled together as another sample for LC-MS/MS analysis. Samples were stored at $-80^{\circ} \mathrm{C}$ until processing. Samples were spiked with 30 ng PGE2-d4, 10,11- DiHN, and 10,11-EpHep (Cayman) as internal standards, mixed with 0.1 vol of $1 \%$ acetic acid in 50\% methanol, and extracted by serial passage through Oasis HLB C18 $3 \mathrm{~mL}$ columns (Waters, Milford, MA, USA). Columns were washed twice with $0.1 \%$ acetic acid in $5 \%$ methanol, and eluted with methanol into glass tubes containing $6 \mu \mathrm{L}$ of $30 \%$ glycerol in methanol. The methanol was then evaporated under a stream of nitrogen gas, and the dried tubes were frozen and stored at $-80^{\circ} \mathrm{C}$ until analysis. Online liquid chromatography of extracted samples was performed with an Agilent 1200 Series capillary HPLC (Agilent Technologies, Santa Clara, CA, USA). Separations were achieved using a Halo C18 column (2.7 mm, 10062.1 mm; MAC-MOD Analytical, Chadds Ford, PA), which was held at 50 ${ }^{\circ} \mathrm{C}$. Mobile phase A was 85:15:0.1 water: acetonitrile: acetic acid. Mobile phase B was 70:30:0.1 acetonitrile: methanol: acetic acid. Flow rate was $400 \mu \mathrm{L} / \mathrm{min}$. Gradient elution was used. Mobile phase percentage B and flow rates were varied as follows: $20 \% \mathrm{~B}$ at $0 \mathrm{~min}$, ramp from 0 to $5 \mathrm{~min}$ to $40 \% \mathrm{~B}$, ramp from 5 to $7 \mathrm{~min}$ to $55 \% \mathrm{~B}$, ramp from 7 to $13 \mathrm{~min}$ to $64 \%$ B. From 13 to $19 \mathrm{~min}$ the column was flushed with $100 \% \mathrm{~B}$ at a flow rate of $550 \mu \mathrm{L} / \mathrm{min}$. Samples were solvated in $50 \mu \mathrm{l}$ of $30 \%$ ethanol. The injection volume was $10 \mu \mathrm{L}$. Samples were analyzed in triplicate. Analyses were performed on an MDS Sciex API 3000 equipped with a TurboIonSpray source (Applied Biosystems). Turbo desolvation gas was heated to $425^{\circ} \mathrm{C}$ at a flow rate of $6 \mathrm{~L} / \mathrm{min}$. Negative ion electrospray ionization tandem mass spectrometry with multiple reaction monitoring was used for detection. 


\section{Effect of PPAR $\gamma$-antagonist (T0070907) on CRH Response in $\mathrm{SEH}^{-/-}$and $\mathrm{sEH}^{+/+} \mathrm{Mice:}$}

Isolated hearts from $\mathrm{sEH}^{-/-}$and $\mathrm{sEH}^{+/+}$mice were stabilized for 30-40 min followed by $15 \mathrm{sec}$ of total occlusion. Recordings of the first CRH (baseline CF, CF tracing, LVDP, HR, RV, PHF and RD) were analyzed for each heart and averaged for each group, as mentioned previously. The PPAR $\gamma$-antagonist, T0070907, was infused at a final concentration of $10 \mu \mathrm{M}$ and $1 \%$ of CF rate for $15 \mathrm{~min}$, after which, another $\mathrm{CRH}$ was induced and the same parameters were recorded and analyzed.

Effect of PPAR $\gamma$-agonist (Rosiglitazone) on CRH in $\mathrm{sEH}^{-/-}$and $\mathrm{SEH}^{+/+}$Mice:

After stabilization, hearts from $\mathrm{sEH}^{-/-}$and $\mathrm{sEH}^{+/+}$mice were subjected to $15 \mathrm{sec}$ of total occlusion. As described above, baseline $\mathrm{CRH}$ was induced in each mouse heart. Then, the PPAR $\gamma$-agonist, rosiglitazone, was infused at a final concentration of $10 \mu \mathrm{M}$ for 15 min, followed by another CRH. CRHs before and after rosiglitazone infusion were analyzed.

\section{Effect of L-NAME on CRH Response in $\mathrm{SEH}^{-/}$and $\mathrm{SEH}^{+/+}$Mice:}

Baseline $\mathrm{CRH}$ was induced in each mouse strain. The non-selective nitric oxide synthase inhibitor L-NAME was infused at a final concentration of $100 \mu \mathrm{M}$ for $15 \mathrm{~min}$, after which, another CRH was induced. CRH responses before and after L-NAME infusion were analyzed and compared.

\section{Statistical and Data Analyses:}


Flow debt (baseline flow rate multiplied by occlusion duration) and repayment volume (the integral of hyperemic area above the baseline flow) were calculated using "the integral relative to baseline" function in the data pad of Lab-Chart 7.0 software. Since absolute coronary flow rates change proportionally with heart mass, the repayment volume and flow debt are presented as $\mathrm{mL} / \mathrm{g}$ wet heart weight, and baseline and peak flow rate data are presented as $\left(\mathrm{mL} . \mathrm{min}^{-1}\right.$.g wet

heart weight ${ }^{-1}$ ). Values are means $\pm \mathrm{SE} ; n$ represents the number of animals. For data analysis, two-tailed unpaired $t$-tests were used for unpaired data analysis, repeated measures ANOVAs were used for populations measured 3 times, and two-way ANOVAs were used to compare data between groups. Differences were considered statistically significant when $\mathrm{p} \leq 0.05$. Post hoc power analyses for the main results in each experiment were calculated using $G *$ Power 3.1.9.2. software (Heinrich Heine, Universität Düsseldorf).

\section{Results}

\section{CRH Response:}

Baseline function of $\mathbf{s E H}{ }^{--}$and $\mathbf{S E H}^{+/+}$mouse hearts: Baseline functional data of isolated mouse hearts were recorded after stabilization (between $30-40 \mathrm{~min}$ ) before starting the experimental protocol. There was no statistically significant difference in body weight, heart weight, heart-to-body weight ratio, baseline coronary flow, LVDP (left-ventricular developed pressure), and heart rate between $\mathrm{sEH}^{+/+}$and $\mathrm{sEH}^{-/}$mice (Table 1).

\section{Role of SEH in CRH:}


Comparison of repayment volume between female and male $\mathrm{sEH}^{+/+}$and $\mathrm{sEH}^{-/}$mice showed no statistically significant differences between female (F) and male (M) $\mathrm{sEH}^{+/+}$mice $(\mathrm{p}>0.05)$ or between female (F) and male $\left(\mathrm{MsEH}^{--}\right)$mice $(\mathrm{p}>0.05)$ (Fig. 1). However, $\mathrm{M}-\mathrm{sEH}^{-1-}$ had more enhanced ( $\mathrm{p}=0.04$, Fig. 1) repayment volume compared to $\mathrm{M}-\mathrm{sEH}^{+/+}$. Also, F-sEH ${ }^{-/}$had more enhanced ( $\mathrm{p}=0.01$, Fig. 1) repayment volume compared to $\mathrm{F}-\mathrm{sEH}^{+/+}$. The post hoc power analysis for repayment volume was $77.7 \%$. Due to the lack of sex difference in CRH response, we used equal ratios of male and female mice from each strain for the remainder of the study. Figure 2 presents $\mathrm{CRH}$ comparison between $\mathrm{sEH}^{+/+}$and $\mathrm{sEH}^{-/}$mice. Repayment volume in $\mathrm{sEH}^{-}$ ${ }^{1-}$ was $28 \%$ higher compared to $\mathrm{sEH}^{+/+}$mice $(7.7 \pm 0.4$ and $6.0 \pm 0.2 \mathrm{~mL} / \mathrm{g}$ respectively, $\mathrm{p}<0.001$, Fig. 2B) and the repayment/debt ratio was also $32 \%$ higher in $\mathrm{sEH}^{-/-}$compared to $\mathrm{sEH}^{+/+}$mice $(2.0 \pm 0.1$ and $1.5 \pm 0.1$ respectively, $\mathrm{p}<0.001$, Fig. 2C). The post hoc power analysis for the repayment volume was 96.8\%. Repayment duration (Fig. 2D), baseline coronary flow (CF), leftventricular developed pressure (LVPD) and heart rate (HR) were not significantly different between the two groups.

\section{Oxylipins Analysis in Heart Perfusate Samples:}

Heart perfusate oxylipin levels were determined by LC-MS/MS. Perfusate samples were collected at baseline after stabilization and after the $15 \mathrm{sec}$ ischemia in $\mathrm{sEH}^{+/+}$and $\mathrm{sEH}^{-/-}$mice. Out of the four regioisomers of EETs, only 14,15-EET and its corresponding metabolite $(14,15$ DHET) were detected. As expected, 14,15-EET was increased by 2.5 fold $(\mathrm{p}<0.001)$, whereas 14,15-DHET was decreased $(\mathrm{p}<0.001)$ in $\mathrm{sEH}^{-/-}$vs. $\mathrm{sEH}^{+/+}$mice at baseline and post-ischemia (Fig. 3A). As a result, the 14,15 -EET/DHET ratio was increased in $\mathrm{sEH}^{-/-}$vs. $\mathrm{sEH}^{+/+}$mice $(\mathrm{p}<0.001$, Fig. 3B). The post hoc power analysis for the 14,15-EET/DHET ratio was $94.5 \%$. 
There was no difference in either metabolite pre- or post-ischemia within the same strain (Fig. 3).

Our LC-MS/MS also detected 4 regioisomers of mid-chain HETEs: 5-, 11-, 12-, and 15HETEs. All detected HETEs (5-, 11-, 12-, and 15-HETE) were significantly decreased in postischemia compared to baseline in both $\mathrm{sEH}^{-/-}$and $\mathrm{sEH}^{+/+}$mice $(\mathrm{p}=0.02, \mathrm{p}=0.04, \mathrm{p}=0.05$, and $\mathrm{p}=0.03$ respectively, Fig. 4A-D), except for 12-HETE in $\mathrm{sEH}^{+/+}$mice, which did not reach significance. There was no difference in any of the measured HETE metabolites between $\mathrm{sEH}^{-/-}$ and $\mathrm{sEH}^{+/+}$mice (Fig. 4A-D). The post hoc power analysis for 5-, 11-, 12-, and 15-HETEs was $56 \%, 48 \%, 48 \%$, and $73 \%$ respectively.

The 9,-10-epoxyoctadecaenoic acid (9,10-EpOME) was increased by $118 \%$ at baseline in $\mathrm{sEH}^{-/}$versus $\mathrm{sEH}^{+/+}$mice (Fig. 5A, $\mathrm{p}=0.03$ ), but not post-ischemia (Fig. 5A, $\mathrm{p}>0.05$ ), whereas 12,13-EpOME was increased by $116 \%$ at baseline and $46 \%$ post-ischemia in $\mathrm{sEH}^{-/}$vs. $\mathrm{sEH}^{+/+}$ mice (Fig. 5A, p=0.03). However, 9,10- and 12,13-DiHOMEs, dihydroxyoctadecaenoic acids, were decreased by $59 \%$ and $77 \%$, respectively, at baseline ( $\mathrm{p}<0.01$ ), and by $48 \%$ and $76 \%$, respectively, post-ischemia (Fig. 5B, $\mathrm{p}<0.001$ ) in $\mathrm{sEH}^{--}$vs. $\mathrm{sEH}^{+/+}$mice. Interestingly, a decreasing trend in EpOMEs post-ischemia compared to baseline was observed in $\mathrm{sEH}^{--}$mice, but was not statistically significant (Fig. 5A, p=0.21). As a result, 9,10-EpOME/DiHOME and 12,13-EpOME/DiHOME ratios were increased at baseline (Fig. 5C, p<0.01), and post-ischemia (Fig. 5C, p<0.01). The post hoc power analysis for 9,10-EpOME/DiHOME and 12,13EpOME/DiHOME ratios were $88 \%$, and $89 \%$ respectively. Additionally, analysis of HODEs showed an increase in 13-HODE, but not 9-HODE, in $\mathrm{sEH}^{-/-}$vs. $\mathrm{sEH}^{+/+}$mice at baseline and 
post-ischemia (Fig. 6, p $<0.01$ ). The post hoc power analysis for 13 -HODE analysis was $79 \%$. There was no statistically significant change in EpOMEs, DiHOMEs, or HODEs pre- and postischemia within the same group (Fig. 5, 6, p>0.05).

\section{Effect of PPAR $\gamma$-antagonist (T0070907) on CRH:}

Administering T0070907 decreased CRH in both $\mathrm{sEH}^{+/+}$and $\mathrm{sEH}^{-/-}$mice (Fig. 7A-C). In sEH ${ }^{+/+}$ mice, repayment volume (RV) was decreased by $25 \%$ (from $5.8 \pm 0.5$ to $4.3 \pm 0.3 \mathrm{~mL} / \mathrm{g}$, p $<0.001$, Fig. 7A), repayment/debt (R/D) ratio was decreased by $14 \%$ (from $1.4 \pm 0.1$ to $1.2 \pm$ $0.1, \mathrm{p}<0.01$, Fig. 7B), repayment duration (RD) was decreased by 54\% (from $2.4 \pm 0.3$ to $1.1 \pm$ 0.2 min, $\mathrm{p}=0.02$ Fig. 7C). Similarly, in $\mathrm{sEH}^{---}$mice, repayment volume was decreased by $33 \%$ (from $7.9 \pm 0.8$ to $5.7 \pm 0.8 \mathrm{~mL} / \mathrm{g}, \mathrm{p}<0.01$, Fig. $7 \mathrm{~A}$ ), repayment/debt ratio was decreased by $32 \%$ (from $2.2 \pm 0.4$ to $1.5 \pm 0.2$, p=0.01, Fig. 7B), and repayment duration was decreased by $54 \%$ (from $2.8 \pm 0.3$ to $1.3 \pm 0.1 \mathrm{~min}, \mathrm{p}<0.01$, Fig. 7C). Comparing $\mathrm{CRH}$ responses between $\mathrm{sEH}^{-/-}$ and $\mathrm{sEH}^{+/+}$mice without T0070907: repayment volume and repayment/debt ratio were increased more in $\mathrm{sEH}^{-/-}$vs. $\mathrm{sEH}^{+/+}$mice $(\mathrm{p}<0.05$ and $\mathrm{p}=0.04$ respectively, Fig. 7A \& 7B). The post hoc power analysis for the repayment volume was $76 \%$. In both $\mathrm{sEH}^{+/+}$and $\mathrm{sEH}^{-/-}$mice, baseline $\mathrm{CF}$, peak PHF, HR and LVDP were not changed by T0070907 (P>0.05, Fig. 7D-F).

\section{Effect of PPAR $\gamma$-agonist (rosiglitazone) on CRH:}

Infusion of rosiglitazone increased $\mathrm{CRH}$ in both $\mathrm{sEH}^{+/+}$and $\mathrm{sEH}^{-/-}$mice. In $\mathrm{sEH}^{+/+}$mice, repayment volume (RV) was increased by $37 \%$ (from $6.1 \pm 0.4$ to $8.4 \pm 1.3 \mathrm{~mL} / \mathrm{g}, \mathrm{p}=0.04$, Fig. 8A), repayment duration $(\mathrm{RD})$ was increased by $38 \%$ (from $2.1 \pm 0.3$ to $2.9 \pm 0.4 \mathrm{~min}, \mathrm{p}=0.05$, Fig. 8C), and baseline coronary flow (CF) was increased by $13 \%$ (from $15.1 \pm 0.2$ to $17.1 \pm 0.9$ 
$\mathrm{mL} / \mathrm{min} / \mathrm{g}, \mathrm{p}=0.03$, Fig. 8D). Although repayment/debt (R/D) ratio was increased by $25 \%$ (from $1.6 \pm 0.1$ to $2.0 \pm 0.4$ ), it was not statistically significant (Fig. $8 B$ ). Likewise, in $\mathrm{sEH}^{-/-}$mice, rosiglitazone increased repayment volume by $37 \%$ (from $7.6 \pm 0.5$ to $10.4 \pm 1.1 \mathrm{~mL} / \mathrm{g}, \mathrm{p}=0.04$, Fig. 8A), repayment duration was increased by $50 \%$ (from $2.0 \pm 0.1$ to $3.0 \pm 0.3 \mathrm{~min}, \mathrm{p}=0.01$, Fig. 8C), and baseline coronary flow (CF) was increased by $9 \%$ (from $14.0 \pm 0.6$ to $15.2 \pm 0.7$ $\mathrm{mL} / \mathrm{min} / \mathrm{g}, \mathrm{p}=0.04$, Fig. 8D). Repayment/debt ratio was also increased by rosiglitazone (from 2.2 \pm 0.1 to $2.7 \pm 0.3$ ), but was not statistically significant (p>0.05, Fig. 8B). Comparison of CRH responses between $\mathrm{sEH}^{-/}$and $\mathrm{sEH}^{+/+}$mice without rosiglitazone: repayment volume and repayment/debt ratio were increased more in $\mathrm{sEH}^{-/-}$vs. $\mathrm{sEH}^{+/+}(\mathrm{p}=0.02$ and $\mathrm{p}=0.05$ respectively, Fig. 8A \& 8B). The post hoc power analysis for the repayment volume was $87 \%$. In both $\mathrm{sEH}^{+/+}$ and $\mathrm{sEH}^{-1-}$ mice, PHF, HR and LVDP were not changed by rosiglitazone (p>0.05, Fig. 8E \& 8F).

\section{Effect of L-NAME on CRH:}

Administering L-NAME decreased $\mathrm{CRH}$ in both $\mathrm{sEH}^{+/+}$and $\mathrm{sEH}^{-/-}$mice. No statistically significant difference was observed between L-NAME-infused $\mathrm{sEH}^{+/+}$and L-NAME-infused $\mathrm{sEH}^{-/}$mice ( $\mathrm{p}=0.36$, Fig. 9A-C) In $\mathrm{sEH}^{+/+}$mice, L-NAME decreased repayment volume (RV) by $43 \%$ (from $6.5 \pm 0.6$ to $3.7 \pm 0.3 \mathrm{~mL} / \mathrm{g}, \mathrm{p}<0.01$, Fig. 9A), decreased repayment duration (RD) by $47 \%$ (from $1.7 \pm 0.2$ to $0.9 \pm 0.1$ min, p<0.01, Fig. 9C), decreased baseline CF by $36 \%$ (from $14.1 \pm 0.7$ to $9.0 \pm 0.9 \mathrm{~mL} / \mathrm{min} / \mathrm{g}, \mathrm{p}<0.001$, Fig. 9D), decreased peak hyperemic flow (PHF) from $34.4 \pm 0.8$ to $31.2 \pm 0.7 \mathrm{~mL} / \mathrm{min} / \mathrm{g}$ (p<0.01, Fig. 9E), and decreased heart rate (HR) from $366.7 \pm$ 10.1 to $338.5 \pm 18.5$ beat/min ( $\mathrm{p}=0.01$, Fig. 9F). Repayment/debt (R/D) ratio (Fig. 9B) and LVDP (data not shown) were not different between the two groups. Similarly, in $\mathrm{sEH}^{-/-}$mice, L- 
NAME decreased repayment volume by $54 \%$ (from $8.9 \pm 1.3$ to $4.1 \pm 0.3 \mathrm{~mL} / \mathrm{g}, \mathrm{p}<0.01$, Fig. 9A), decreased repayment/debt ratio by $26 \%$ (from $2.7 \pm 0.3$ to $2.0 \pm 0.1, p=0.03$, Fig. 8B), decreased repayment duration by $61 \%$ (from $2.3 \pm 0.2$ to $0.9 \pm 0.1 \mathrm{~min}, \mathrm{p}<0.001$, Fig. 9C), and decreased baseline CF by $36 \%$ (from $13.5 \pm 0.9$ to $8.6 \pm 0.9 \mathrm{~mL} / \mathrm{min} / \mathrm{g}, \mathrm{p}<0.001$, Fig. 9D), PHF, HR and LVPD were not significantly changed by L-NAME (Fig. 9E \& F). Comparing CRH responses between $\mathrm{sEH}^{-/-}$and $\mathrm{sEH}^{+/+}$mice without L-NAME treatment: repayment volume and repayment duration were increased more in $\mathrm{sEH}^{-/-}$vs. $\mathrm{sEH}^{+/+}$mice $(\mathrm{p}=0.01$ and $\mathrm{p}=0.01$ respectively, Fig. 9A \& C). The post hoc power analysis for the repayment volume was $97 \%$. We summarized our observed results into a proposed schematic diagram (Fig. 10).

Tables, Figures, and Figure Legends:

\begin{tabular}{|c|c|c|}
\hline & $\mathrm{SEH}^{+/+}$ & $\mathbf{S E H}{ }^{-/-}$ \\
\hline Age, weeks & $14.6 \pm 0.3$ & $14.7 \pm 0.4$ \\
\hline Body weight, g & $22.7 \pm 1.1$ & $24.3 \pm 1.5$ \\
\hline Heart weight, mg & $109 \pm 7.0$ & $121 \pm 10.0$ \\
\hline Heart-to-body weight ratio, \% & $0.48 \pm 0.01$ & $0.49 \pm 0.01$ \\
\hline Coronary flow, ml.min ${ }^{-1} \cdot \mathrm{g}^{-1}$ & $15.7 \pm 0.6$ & $16.5 \pm 0.8$ \\
\hline LVDP, mmHg & $109.6 \pm 10.0$ & $115.0 \pm 12.3$ \\
\hline Heart rate, beat.min ${ }^{-1}$ & $414 \pm 11$ & $396 \pm 6$ \\
\hline
\end{tabular}

Table 1.1: Baseline functional data for wild type $\left(\mathrm{sEH}^{+/+}\right)$and $\mathrm{sEH}-\mathrm{null}\left(\mathrm{sEH}^{--}\right)$isolated mouse heart. $n=12$ per group. 


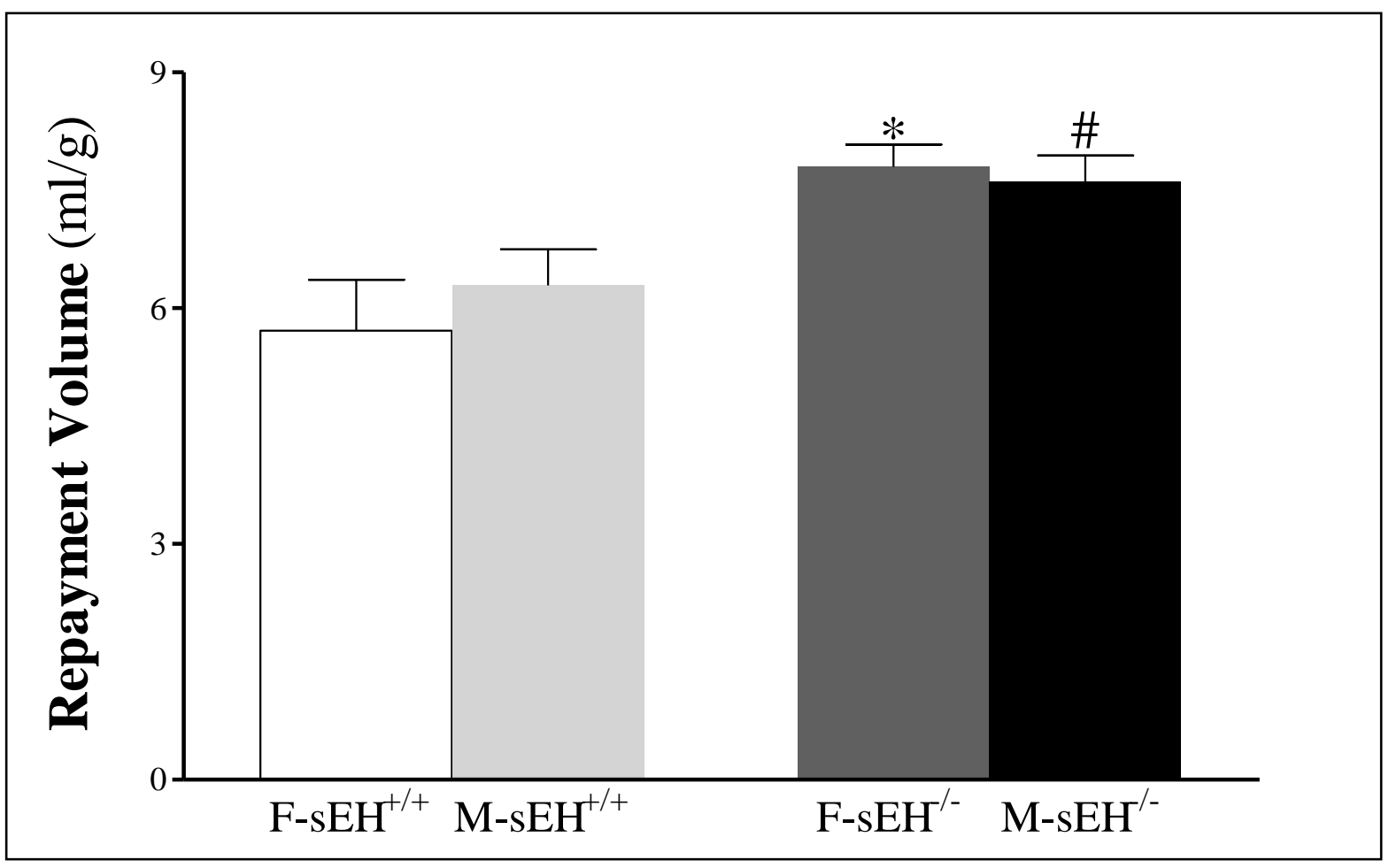

Figure 1.1: Comparison of repayment volume (RV) between female and male wild type $\left(\mathrm{sEH}^{+/+}\right)$ and $\mathrm{sEH}^{-1-}$ (sEH-null) mice. Repayment volume was not significantly different between female $\left(\mathrm{F}_{-} \mathrm{SEH}^{+/+}\right)$and male $\left(\mathrm{M}-\mathrm{sEH}^{+/+}\right)$WT mice $(\mathrm{p}>0.05)$ and between female $\left(\mathrm{F}_{-}-\mathrm{sEH}^{-/}\right)$and male $(\mathrm{M}-$ $\left.\mathrm{sEH}^{--}\right) \mathrm{sEH}-$ null mice $(\mathrm{p}>0.05)$. However, $\mathrm{M}-\mathrm{sEH}^{--}$had more enhanced $(\mathrm{p}=0.04)$ repayment volume compared to $\mathrm{M}-\mathrm{sEH}^{+/+}$. Also, $\mathrm{F}_{-} \mathrm{sEH}^{-/}$had more enhanced $(\mathrm{p}=0.01)$ repayment volume compared to F-sEH ${ }^{+/} .{ }^{*} \mathrm{p} \leq 0.05$ vs. baseline $\mathrm{F}-\mathrm{sEH}^{+/+}$. $\# \mathrm{p} \leq 0.05$ vs. $\mathrm{M}$-sEH ${ }^{+/+} . n=6$ per group. 


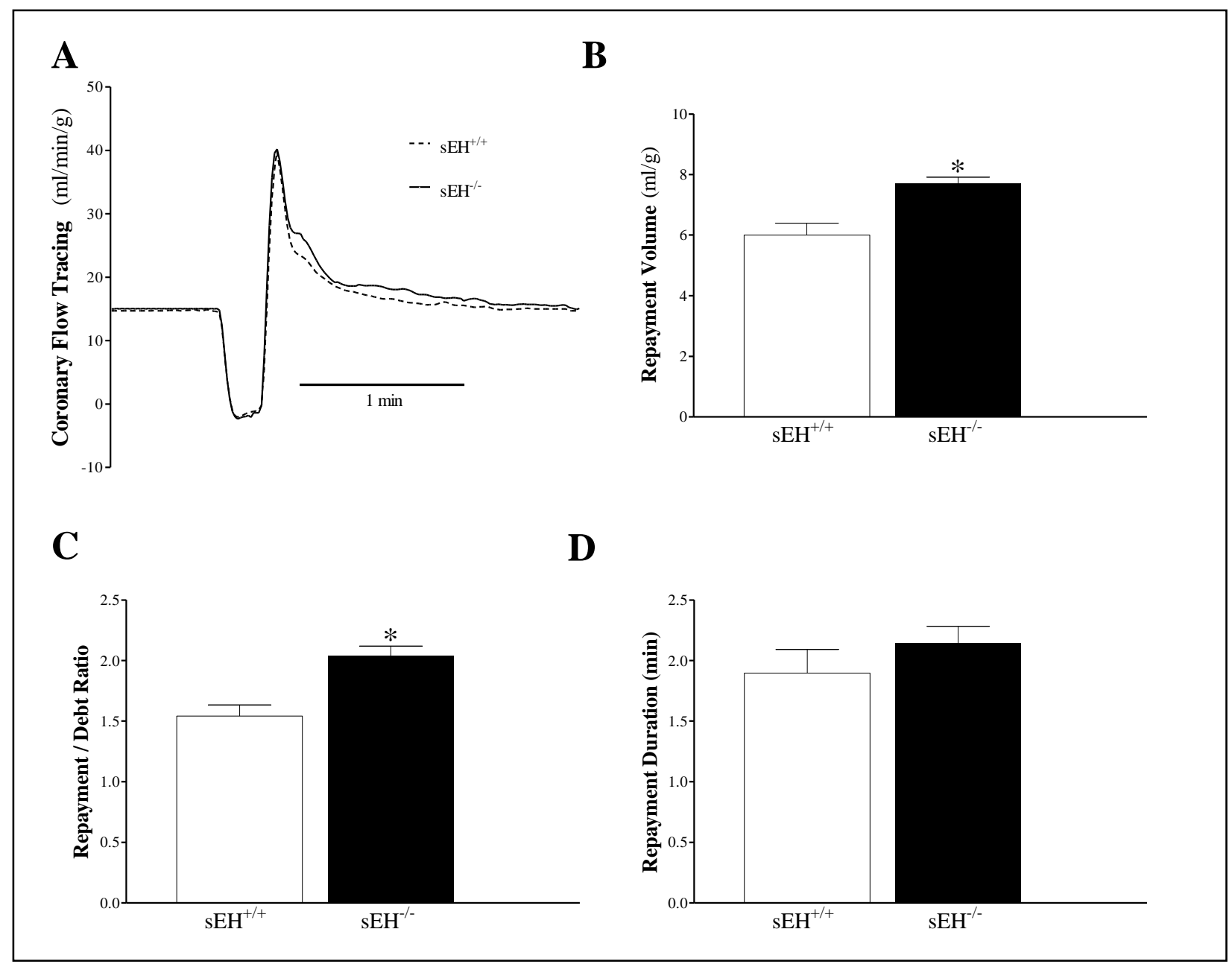

Figure 1.2: Comparison of coronary reactive hyperemia $(\mathrm{CRH})$ between wild type $\left(\mathrm{sEH}^{+/+}\right)$and $\mathrm{sEH}^{-/-}$(sEH-null) mice. (A) tracing depicting coronary flow changes at baseline (before CRH) and after $\mathrm{CRH}$ was induced by 15 -second no-flow ischemia in both $\mathrm{sEH}^{+/+}$(dash line) and $\mathrm{sEH}^{-/-}$ (continuous line) mice. Repayment volume $(\mathrm{B}, \mathrm{p}<0.001)$ and repayment/debt ratio $(\mathrm{C}, \mathrm{p}=0.0004)$ were enhanced in $\mathrm{sEH}^{-/-}$vs. $\mathrm{sEH}^{+/+}$mice. Repayment duration (D) was increased in $\mathrm{sEH}^{-/-}$vs. $\mathrm{sEH}^{+/+}$, but didn't reach statistical significance $(\mathrm{p}=0.31)$. Peak hyperemic flow, baseline CF, leftventricular developed pressure (LVDP) and heart rate (HR) were not significantly different between the two groups (data not shown). ${ }^{*} \mathrm{p} \leq 0.05 \mathrm{sEH}^{+/+} . n=12$ per group. 


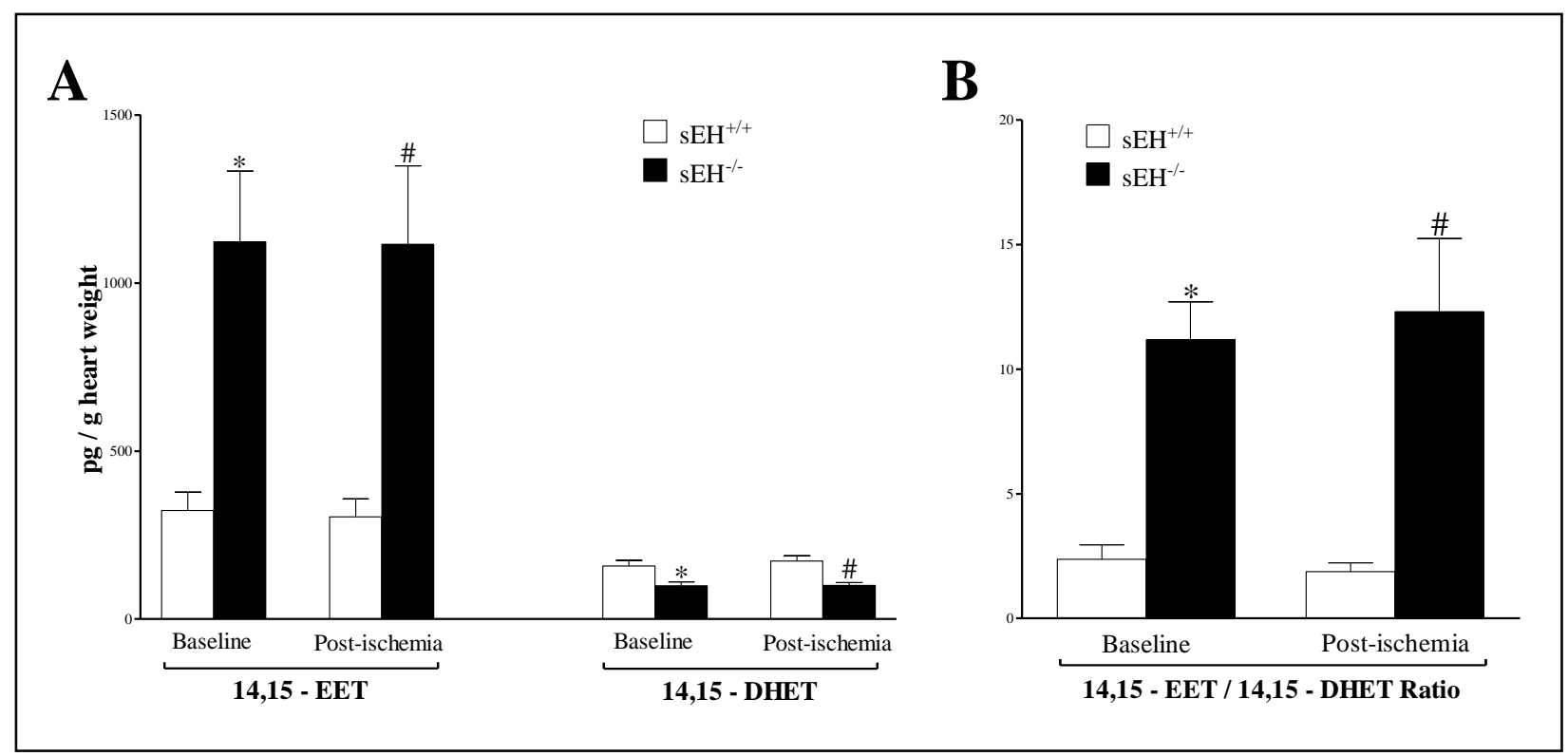

Figure 1.3: LC - MS/MS analysis for 14, 15 - EETs and 14,15-DHETs in $\mathrm{sEH}^{+/+}$and $\mathrm{sEH}^{-/}$ mice heart perfusate at baseline (pre-ischemia) and directly after 15-second ischemia (postischemia). (A) At baseline and post-ischemia, 14,15-EET was increased 2.5 fold in $\mathrm{sEH}^{-/-}$vs. $\mathrm{sEH}^{+/+}$mice $(\mathrm{p}<0.001)$ whereas 14,15 -DHET was decreased $(\mathrm{p}<0.001)$. There was no difference in either metabolite pre- and post-ischemia within the same strain. (B) Ratio between 14,15-EET to $14,15-\mathrm{DHET}$ was increased at baseline and post-ischemia in $\mathrm{sEH}^{-/-}$vs. $\mathrm{sEH}^{+/+}$mice $(\mathrm{p}<0.001)$. ${ }^{*} \mathrm{p} \leq 0.05$ vs. baseline $\mathrm{sEH}^{+/+} . \# \mathrm{p} \leq 0.05$ vs. post-ischemia $\mathrm{sEH}^{+/+} . n=7 \mathrm{sEH}^{+/+}, n=10 \mathrm{sEH}^{-/-}$. 


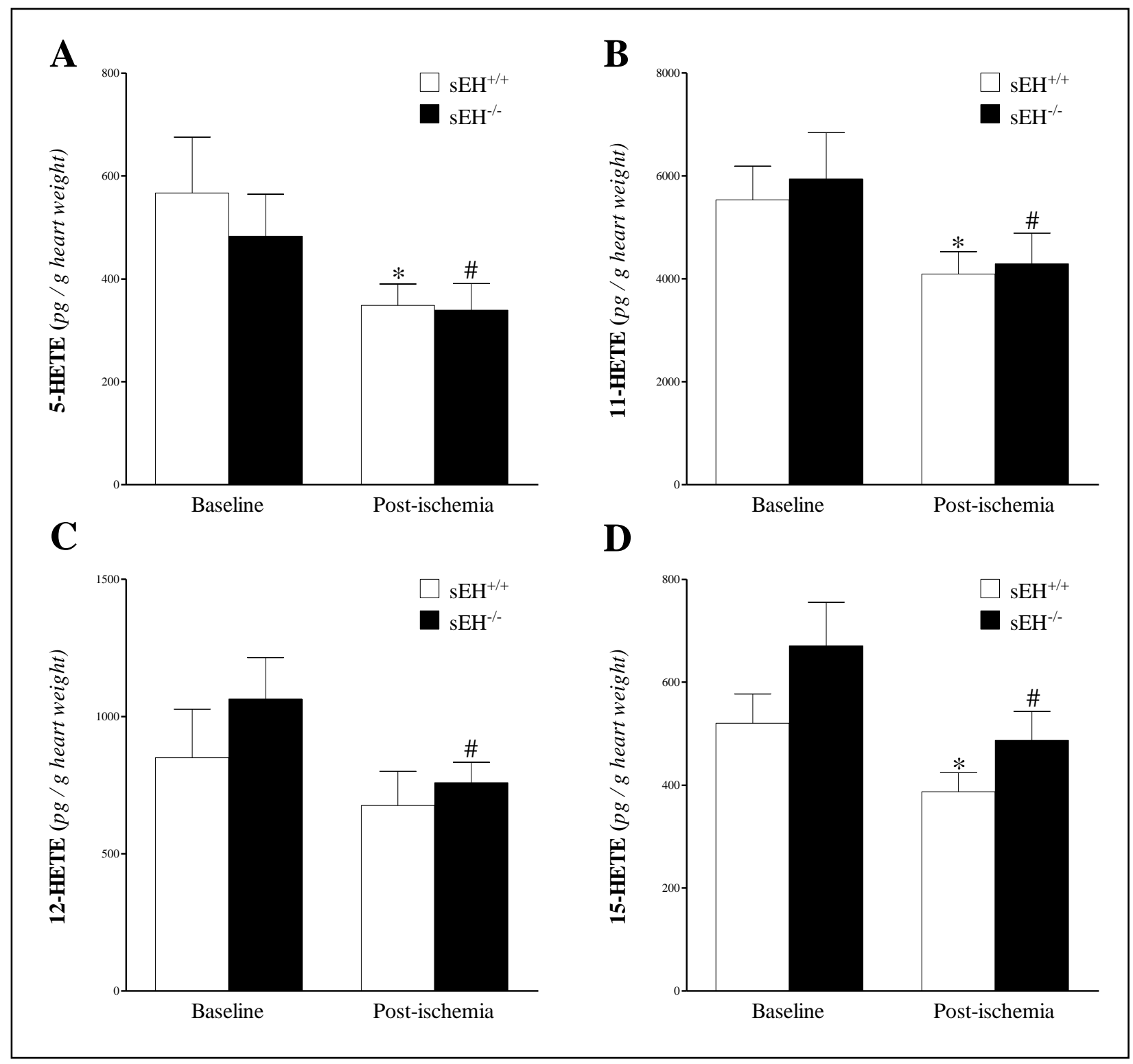

Figure 1.4: $\mathrm{LC}$ - MS/MS analysis of HETEs in $\mathrm{sEH}^{+/+}$and $\mathrm{sEH}^{-/-}$heart perfusate at baseline (pre-ischemia) and directly after 15-second ischemia (post-ischemia). All detected HETEs (5-, 11-, 12-, and 15-HETE), were decreased post-ischemia (after perfusion was reinstated) compared to baseline in both $\mathrm{sEH}^{+/+}$and $\mathrm{sEH}^{-/-}$mice $(\mathrm{p}=0.02, \mathrm{p}=0.04, \mathrm{p}=0.05, \mathrm{p}=0.03$ respectively, A-D). Only 12-HETE in $\mathrm{sEH}^{+/+}$mice did not reach statistically significant level $(\mathrm{p}=0.09)$. There was no difference in any of the measured HETEs between $\mathrm{sEH}^{+/+}$and $\mathrm{sEH}^{-/}$mice $(\mathrm{p}>0.05){ }^{*} \mathrm{p} \leq 0.05 \mathrm{vs}$. baseline $\mathrm{sEH}^{+/+} . \# \mathrm{p} \leq 0.05$ vs. baseline $\mathrm{sEH}^{-/-} . n=7 \mathrm{sEH}^{+/+}, n=10 \mathrm{sEH}^{-/-}$. 
A

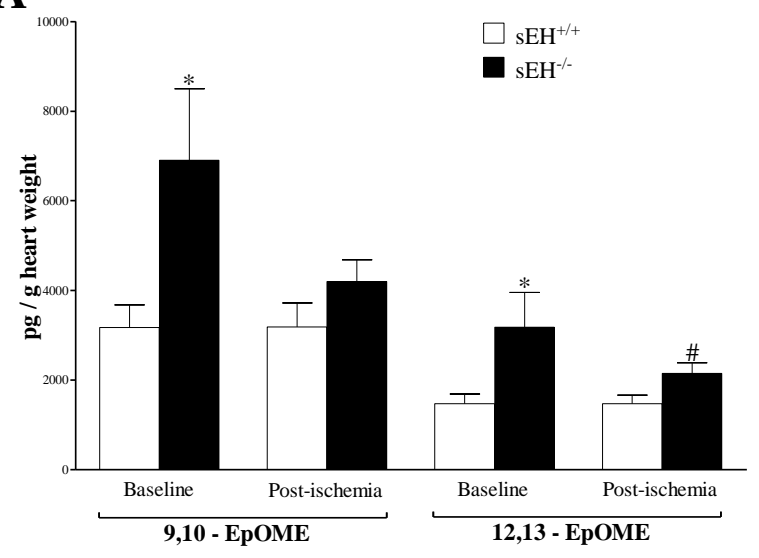

C

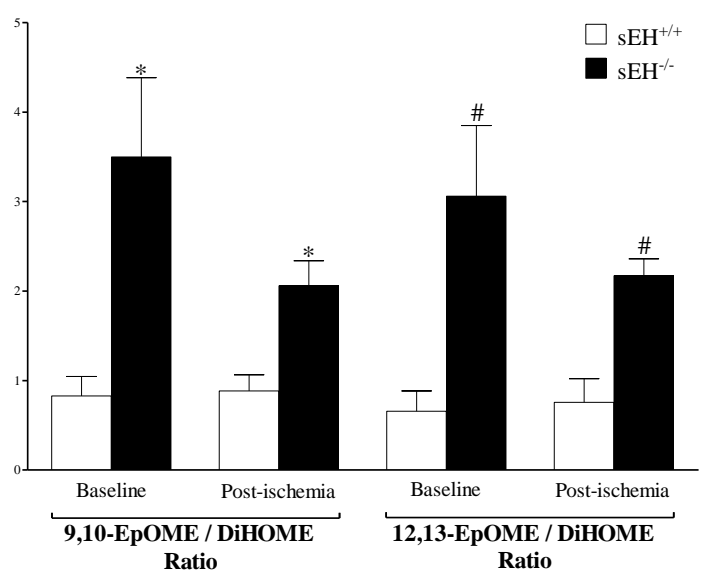

B

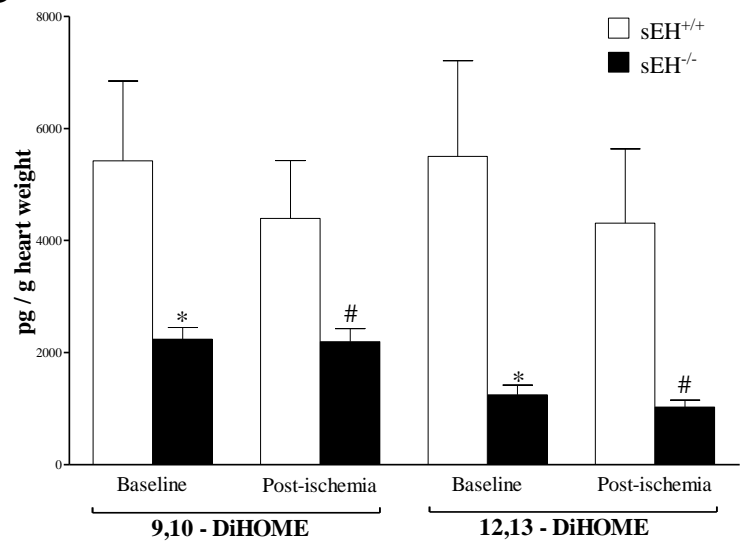

Figure 1.5: LC-MS/MS analysis of EpOMEs and DiHOMEs in $\mathrm{sEH}^{+/+}$and $\mathrm{sEH}^{-/-}$mouse heart perfusate samples at baseline (pre-ischemia) and directly after 15-second ischemia (postischemia). (A) 9,10-EpOME was increased at baseline $(\mathrm{p}=0.03)$ and post-ischemia, but was not statistically significant $(\mathrm{p}=0.21)$, whereas 12,13 -EpOME was increased both at baseline and postischemia ( $\mathrm{p}=0.03$ ) in $\mathrm{sEH}^{-/-}$vs. $\mathrm{sEH}^{+/+}$mice. (B) 9,10- and 12,13-DiHOMEs were decreased at baseline $(\mathrm{p}=0.001)$, and post-ischemia $(\mathrm{p}<0.001)$ in $\mathrm{sEH}^{-/-}$vs. $\mathrm{sEH}^{+/+}$mice. A decreasing trend in EpOMEs post-ischemia compared to baseline was observed in $\mathrm{sEH}^{-/-}$mice, but was not statistically significant. (C) 9,10- and 12,13-EpOME/DiHOME ratios were increased at baseline $(\mathrm{p}=0.002)$, and post-ischemia $(\mathrm{p}=0.0008) .{ }^{*} \mathrm{p} \leq 0.05$ vs. baseline $\mathrm{sEH}^{+/+}$. \# $\mathrm{p} \leq 0.05$ vs. postischemia $\mathrm{sEH}^{+/+} . n=7 \mathrm{sEH}^{+/+}, n=10 \mathrm{sEH}^{-/-}$. 


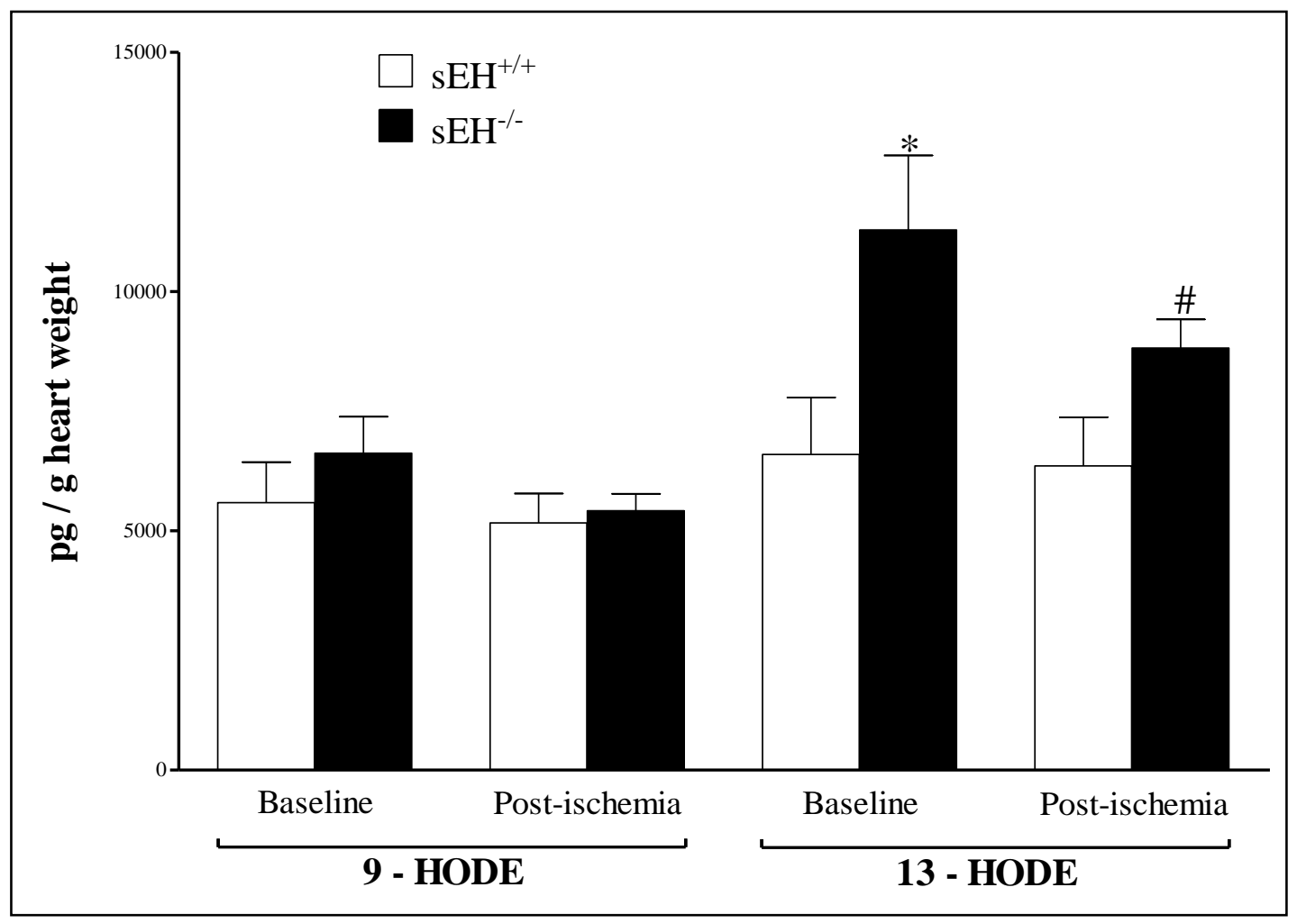

Figure 1.6: LC-MS/MS analysis of HODEs in $\mathrm{sEH}^{+/+}$and $\mathrm{sEH}^{-/}$mouse heart perfusate samples at baseline (pre-ischemia) and directly after 15-second ischemia (post-ischemia). Baseline and post-ischemia analysis 9- and 13-HODEs in $\mathrm{sEH}^{-/}$vs. $\mathrm{sEH}^{+/+}$. 13-HODE, but not 9-HODE, was increased in $\mathrm{sEH}^{-/-}$vs. $\mathrm{sEH}^{+/+}$mice at baseline and post-ischemia $(\mathrm{p}=0.006)$. There was no change in either HODEs before and after ischemia in both groups $(\mathrm{p}>0.05) .{ }^{*} \mathrm{p} \leq 0.05$ vs. baseline $\mathrm{sEH}^{+/+} . \# \mathrm{p} \leq 0.05$ vs. post-ischemia $\mathrm{sEH}^{+/+} . n=7 \mathrm{sEH}^{+/+}, n=10 \mathrm{sEH}^{-/}$. 


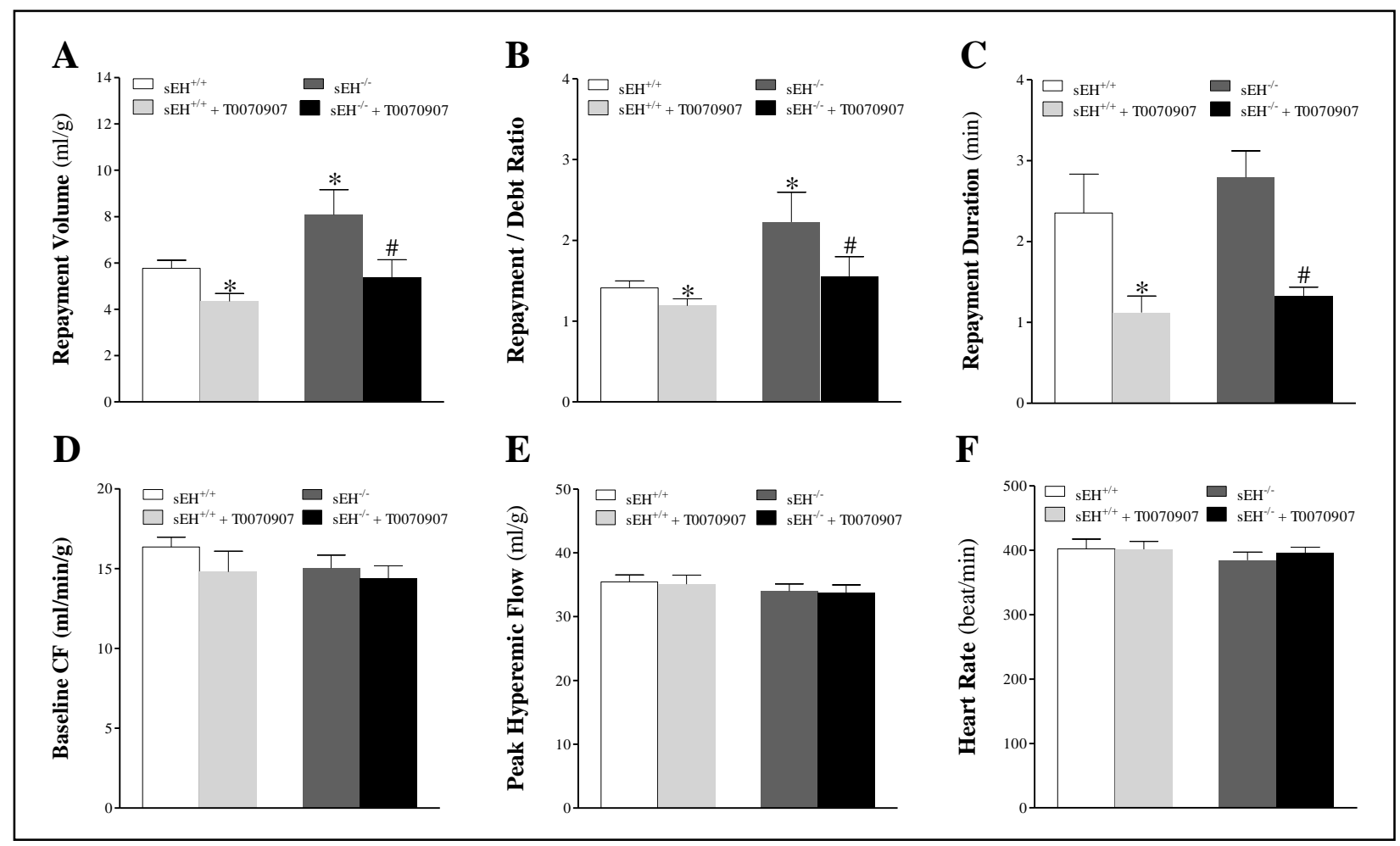

Figure 1.7: Effect of PPAR $\gamma$-antagonist $(\mathrm{T} 0070907,10 \mu \mathrm{M})$ on $\mathrm{CRH}$ in $\mathrm{sEH}^{+/+}$and $\mathrm{sEH}^{-/-}$mice. Infusion of T0070907 decreased CRH in both $\mathrm{sEH}^{+/+}$and $\mathrm{sEH}^{-/-}$mice. In $\mathrm{sEH}^{+/+}$mice, T0070907 decreased repayment volume $(\mathrm{RV})$ by $25 \%(\mathrm{~A}, \mathrm{p}<0.001)$, repayment/debt $(\mathrm{R} / \mathrm{D})$ ratio by $14 \%(\mathrm{~B}$, $\mathrm{p}=0.004)$, and repayment duration $(\mathrm{RD})$ by $54 \%(\mathrm{C}, \mathrm{p}=0.02) . \mathrm{In}_{\mathrm{SEH}}^{-{ }^{-}}$, T0070907 decreased RV by $33 \%(\mathrm{~A}, \mathrm{p}=0.0039)$, repayment/debt ratio by $32 \%(\mathrm{~B}, \mathrm{p}=0.01)$, and $\mathrm{RD}$ by $54 \%(\mathrm{C}, \mathrm{p}=0.003)$. Comparing $\mathrm{sEH}^{-/-}$and $\mathrm{sEH}^{+/+}$mice: repayment volume and repayment/debt ratio were increased more in $\mathrm{sEH}^{-/-}$vs. $\mathrm{sEH}^{+/+}$mice $\left(\mathrm{A}, \mathrm{p}=0.048\right.$ and $\mathrm{B}, \mathrm{p}=0.04$ respectively). In both $\mathrm{sEH}^{+/+}$and $\mathrm{sEH}^{-/-}$mice, baseline CF (D), PHF (E), HR (F), and LVDP (data not shown) were not changed by $\mathrm{T} 0070907 .{ }^{*} \mathrm{p} \leq 0.05$ vs. $\mathrm{sEH}^{+/+} . \# \mathrm{p} \leq 0.05$ vs. $\mathrm{sEH}^{-/-} . n=6$ per group. 


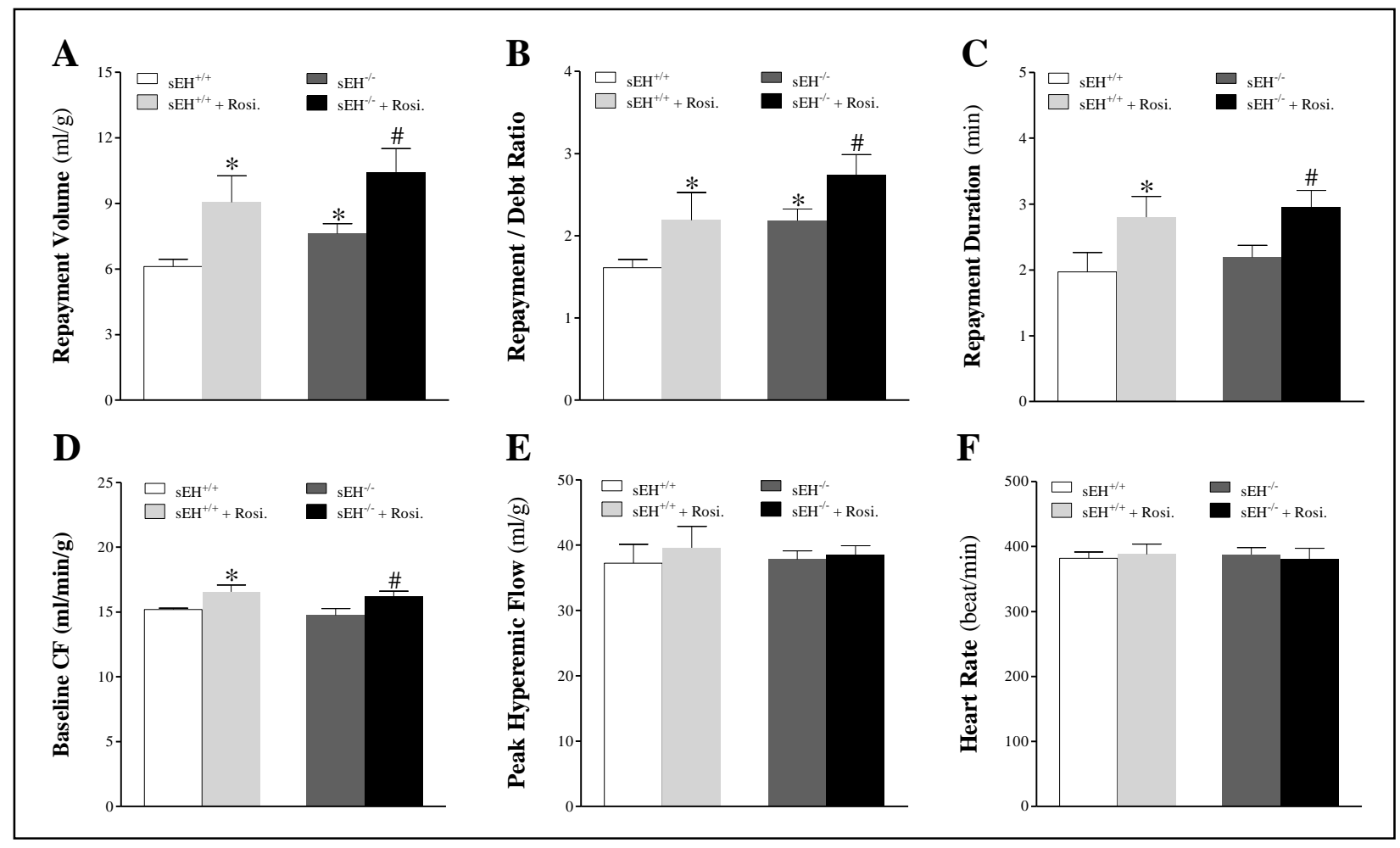

Figure 1.8: Comparison $\mathrm{CRH}$ for wild type $\left(\mathrm{sEH}^{+/+}\right)$mice before and after infusion of rosiglitazone (PPAR $\gamma$-agonist, $10 \mu \mathrm{M}$ ); administration of rosiglitazone increased $\mathrm{CRH}$ in both $\mathrm{sEH}^{+/+}$and $\mathrm{sEH}^{-/-}$mice. In $\mathrm{sEH}^{+/+}$mice, repayment volume $(\mathrm{RV})$ was increased by $37 \%(\mathrm{~A}$, $\mathrm{p}=0.04)$, repayment/debt ratio $(\mathrm{R} / \mathrm{D})$ by $38 \%(\mathrm{~B}, \mathrm{p}=0.03)$, repayment duration $(\mathrm{RD})$ by $38 \%(\mathrm{C}$, $\mathrm{p}=0.05)$, and baseline coronary flow $(\mathrm{CF})(\mathrm{D}, \mathrm{p}=0.0280)$. In $\mathrm{sEH}^{-/-}$mice, rosiglitazone increased RV by $37 \%$ (A, p=0.04), repayment/debt ratio (R/D) by $23 \%(\mathrm{~B}, \mathrm{p}=0.03), \mathrm{RD}$ by $50 \%(\mathrm{C}$, $\mathrm{p}=0.01)$, and baseline coronary flow $(\mathrm{CF})(\mathrm{D}, \mathrm{p}=0.04)$. Comparing $\mathrm{sEH}^{-/-}$and $\mathrm{sEH}^{+/+}$mice: $\mathrm{RV}$ and $\mathrm{R} / \mathrm{D}$ were increased more in $\mathrm{sEH}^{-/-}$vs. $\mathrm{sEH}^{+/+}$mice $(\mathrm{p}=0.02$ and $\mathrm{p}=0.05$ respectively). In both $\mathrm{sEH}^{+/+}$and $\mathrm{sEH}^{-/-}$mice, PHF (E), HR (F), and LVDP (data not shown) were not significantly changed by rosiglitazone. ${ }^{*} \mathrm{p} \leq 0.05 \mathrm{vs} . \mathrm{sEH}^{+/+} . \# \mathrm{p} \leq 0.05 \mathrm{vs} . \mathrm{sEH}^{-/-} . n=6 \mathrm{sEH}^{+/+}, n$ $=7 \mathrm{sEH}^{-1-}$. 


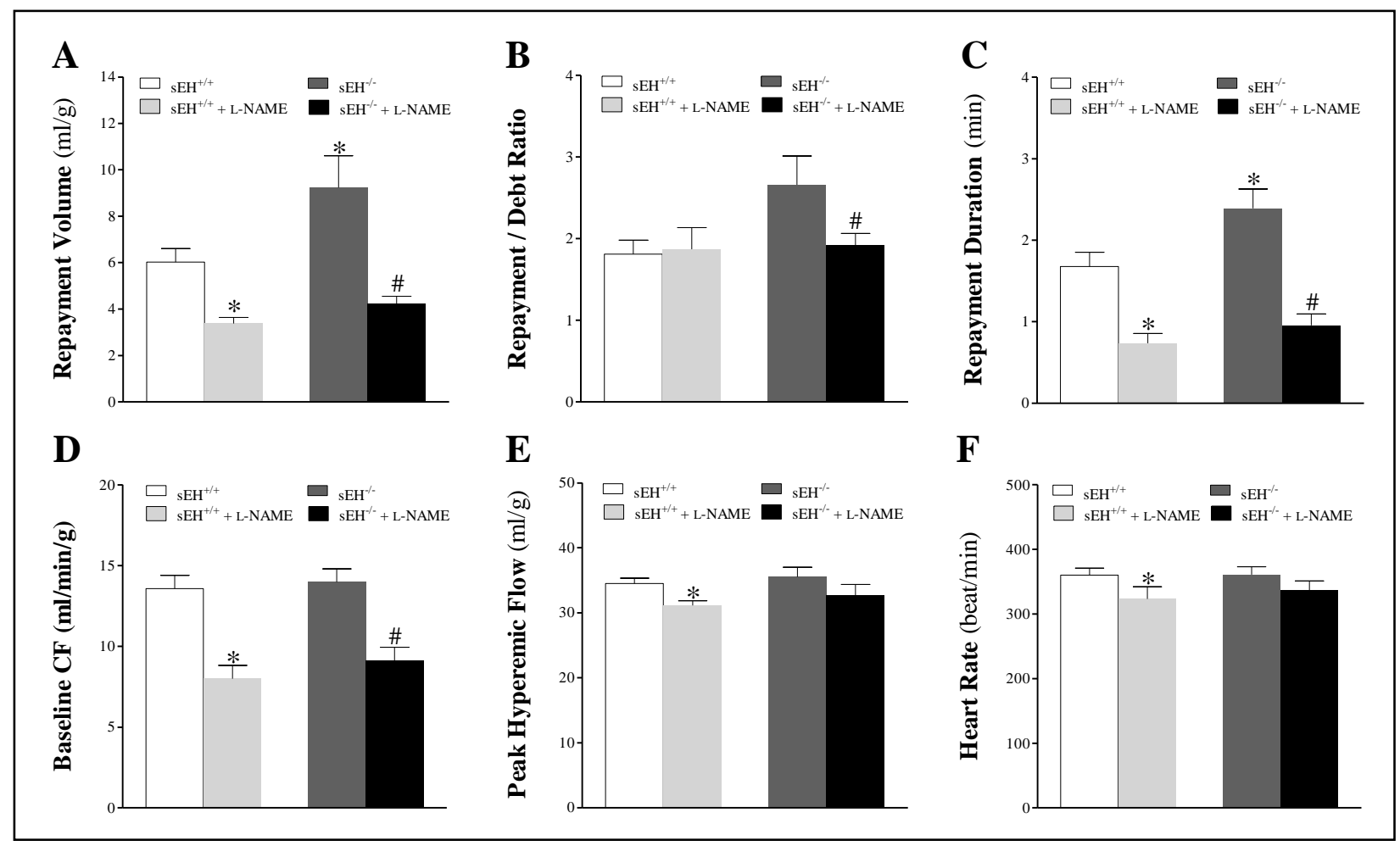

Figure 1.9: Comparison of the effect of L-NAME (nitric oxide synthase inhibitor) on coronary reactive hyperemia $(\mathrm{CRH})$ in $\mathrm{sEH}-$ null $\left(\mathrm{sEH}^{-/}\right)$and $\mathrm{sEH}^{+/+}$mice before and after infusion of LNAME $(100 \mu \mathrm{M})$. L-NAME decreased $\mathrm{CRH}$ in both $\mathrm{sEH}^{+/+}$and $\mathrm{sEH}^{-/}$mice, and $\mathrm{CRH}$ responses between the two groups were not statistically significant $(\mathrm{p}=0.36)$. In $\mathrm{sEH}^{+/+}$, L-NAME decreased repayment volume $(\mathrm{RV})$ by $43 \%$ (A, $\mathrm{p}=0.002)$, repayment duration $(\mathrm{RD})$ by $47 \%(\mathrm{C}$, $\mathrm{p}=0.002)$, baseline $\mathrm{CF}$ by $36 \%(\mathrm{D}, \mathrm{p}<0.001)$, peak hyperemic flow $(\mathrm{PHF})(\mathrm{E}, \mathrm{p}=0.001)$, and heart rate $(\mathrm{HR})(\mathrm{F}, \mathrm{p}=0.01)$. Repayment / debt (R/D) ratio (B) and LVDP (not shown) were not different between the two groups. In $\mathrm{sEH}^{-/-}$mice, L-NAME decreased $\mathrm{RV}$ by $54 \%(\mathrm{~A}, \mathrm{p}=0.005)$, $\mathrm{R} / \mathrm{D}$ ratio by $26 \%(\mathrm{~B}, \mathrm{p}=0.03), \mathrm{RD}$ by $61 \%(\mathrm{C}, \mathrm{p}<0.001)$, and baseline $\mathrm{CF}$ by $36 \%(\mathrm{D}, \mathrm{p}<0.001)$, PHF (E), HR (F) and LVPD (not shown) were not significantly changed by L-NAME. RV and $\mathrm{RD}$ were increased more in untreated $\mathrm{sEH}^{-/-}$vs. untreated $\mathrm{sEH}^{+/+}$mice $(\mathrm{A}, \mathrm{p}=0.01$ and $\mathrm{C}, \mathrm{p}=0.01$ respectively). $n=8$ per group. 

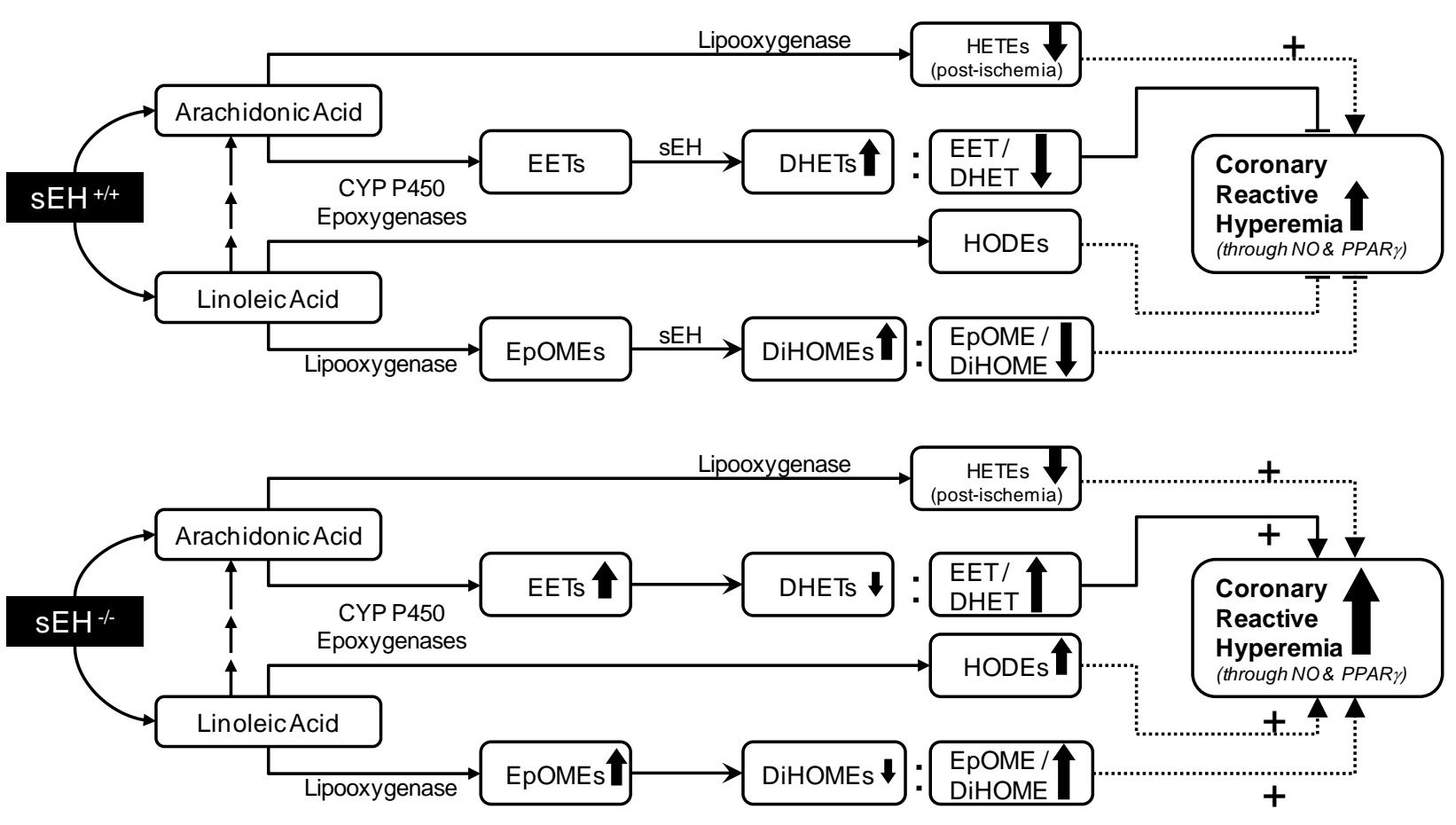

Figure 1.10: A schematic diagram comparing the changes observed for selected oxylipins in response to short-lived ischemia and their possible impact on coronary reactive hyperemia $(\mathrm{CRH})$ between $\mathrm{sEH}^{-/-}$and $\mathrm{sEH}^{+/+}$mice. $\mathrm{sEH}$ deletion enhanced $\mathrm{CRH}$ possibly through increased 14,15-EET/DHET ratio, increased 13-HODE, increased EpOME/DiHOME ratio. Role of NO, PPAR $\gamma$ and mid-chain HETEs was comparable in both $\mathrm{sEH}^{-/}$and $\mathrm{sEH}^{+/+}$mice. 


\section{Discussion}

Global deletion of soluble epoxide hydrolase $\left(\mathrm{sEH}^{--}\right)$in mice shows an increase in coronary reactive hyperemia $(\mathrm{CRH})$ in isolated heart model after a brief ischemia. The observed changes in oxylipins associated with sEH-deletion (increased EETs/DHETs, increased EpOMEs/DiHOMEs, and increased HODEs) could be involved in enhancing CRH. The relationship between sEH deletion, oxylipins, $\operatorname{PPAR} \gamma$, and $\mathrm{CRH}$ in isolated mouse hearts is unknown. Therefore, we designed this study to investigate the role of sEH-deletion, and the associated changes in various oxylipins, in the modulation of $\mathrm{CRH}$ using $\mathrm{sEH}^{-/}$and $\mathrm{sEH}^{+/+}$ mice. Our data demonstrate that, compared with $\mathrm{sEH}^{+/+}, \mathrm{sEH}^{-/-}$mice had: 1) increased $\mathrm{CRH}$; 2) increased 14,15-EET/DHET ratio; 3) increased EpOMEs/DiHOMEs ratio; and 4) increased 13HODE. Also, both $\mathrm{sEH}^{+/+}$and $\mathrm{sEH}^{-/-}$mice had: 1) comparable role of PPAR $\gamma$ in mediating $\mathrm{CRH}$; 2) similar response to L-NAME-mediated decrease in $\mathrm{CRH}$; and 3) similar decrease in mid-chain HETEs post-ischemia.

In this study, $\mathrm{sEH}$ deletion in mice $\left(\mathrm{sEH}^{--}\right.$mice) was associated with a statistically significant increase in $\mathrm{CRH}$ after a brief ischemia compared to $\mathrm{SEH}^{+/+}$mice. Interestingly, there was no difference in $\mathrm{CRH}$, as measured by repayment volume, between male and female mice in either strain $\left(\mathrm{sEH}^{-/-}\right.$and $\left.\mathrm{sEH}^{+/+}\right)$. This contrasts to the sex difference in vascular response between male $\left(\mathrm{M}-\mathrm{sEH}^{+/+}\right)$and female $\left(\mathrm{F}-\mathrm{sEH}^{+/+}\right)$wild type mice, where $\mathrm{F}-\mathrm{sEH}^{+/+}$exhibited increased flow/shear stress-induced dilator response (FID) in isolated gracilis muscle arterioles compared to $\mathrm{M}-\mathrm{sEH}^{+/+}$(73); In addition, Qin et al., (73) reported that sEH deletion in male $\left(\mathrm{M}-\mathrm{sEH}^{-/-}\right)$ enhanced FID to a level comparable to that observed in $\mathrm{F}_{-} \mathrm{sEH}^{-/}$and female $\mathrm{F}-\mathrm{sEH}^{+/+}$. Therefore, the authors suggested that deletion of sEH abolished the sex difference in FID 
response observed in wild type mice (73). But, in our study, we did not observe any sex difference in $\mathrm{CRH}$ response. Although we used the same mouse strains $\left(\mathrm{sEH}^{-/}\right.$and $\left.\mathrm{sEH}^{+/+}\right)$Qin et al. used in their study, the lack of sex difference in CRH response in our study was probably due to different tissues studied (isolated mouse heart versus leg gracilis muscle's second order arterioles (73)) and the different triggering factor for vasodilation (CRH in response to ischemia versus FID (73)).

$\mathrm{CRH}$ may be viewed as a protective mechanism, and is attenuated in pathologic conditions. For example, CRH is decreased in cardiac hypertrophy (36), metabolic syndrome (6), unstable angina, myocardial infarction, congestive heart failure and cardiac death (30). Out of the four EET isomers, only 14,15-EET was detected in the heart perfusate samples by LCMS/MS. The other EET isomers (5,6-, 8,9-, and 11,12-EETs) were not detected possibly because their level was lower than the level of 14,15-EET and lower than the detection power of the LC-MS/MS. In support of this speculation, Li et al. (45) reported that the level of 14,15-EET was approximately 3 time higher than that of 5,6-, 8,9-, and 11,12-EETs isomers in mouse plasma samples. Our results demonstrate that in $\mathrm{sEH}^{-/}$mice $14,15-\mathrm{EET} / \mathrm{DHET}$ ratio was increased. EETs are well established as substrates for sEH $(55,62)$. Also, in the presence of sEH, EETs are rapidly converted by hydration to their corresponding DHETs resulting in loss of the beneficial cardiovascular effects of these epoxyeicosanoids $(53,59,85,102)$. Evidently, disruption of sEH causes EETs to accumulate, be retained for longer periods, and to continue to exert their beneficial effects $(19,59)$. Besides the main, sEH-catalyzed, breakdown pathway, EETs can be broken down through other minor pathways, such as $\omega$-oxidation, $\beta$-oxidation, and chain elongation. The latter two pathways become more important when sEH activity is inhibited or deleted (32). None of these minor pathways has been studied or considered in our 
study. EETs also exert cardioprotective effects in ischemia/reperfusion injury (77). Deletion of sEH gene increases endogenous EETs by decreasing the metabolic conversion of EETs into the less-active DHETs. Our results suggest that CRH increases in mice due to sEH deletion, which is shown here to be associated with an increase in 14,15-EET/14,15-DHET ratio without changing baseline coronary flow, heart rate, and left-ventricular developed pressure.

This study also showed that $\mathrm{CRH}$ in $\mathrm{sEH}^{+/+}$and $\mathrm{sEH}^{-/-}$mouse hearts was attenuated by T0070907 (PPAR $\gamma$-antagonist), but enhanced by rosiglitazone (PPAR $\gamma$-agonist). These results from PPAR $\gamma$-antagonist and PPAR $\gamma$-agonist experiments suggest that PPAR $\gamma$ receptors are involved in modulating CRH. Interestingly, our lab reported that $\mathrm{sEH}^{-/}$mouse aorta had increased expression of PPAR $\gamma$ protein and enhanced vascular relaxation compared to $\mathrm{sEH}^{+/+}$ mice (59). Liu et al. suggested that selective $\mathrm{sEH}$ inhibition potentiated the anti-inflammatory effect in endothelial cells, presumably by increasing the retention of EETs, which would activate PPAR $\gamma(46)$. These studies indicate that EETs' effects could be mediated by PPAR $\gamma$ receptors. However, our results did not show the same relationship; the effect of T0070907 and rosiglitazone were comparable between $\mathrm{sEH}^{+/+}$and $\mathrm{sEH}^{-/-}$mice. Therefore, we found that $\mathrm{CRH}$ in $\mathrm{sEH}^{+/+}$and $\mathrm{sEH}^{-/-}$mice may similarly be mediated by PPAR $\gamma$.

Infusion of L-NAME, a non-selective NO synthase inhibitor, decreased CRH in both $\mathrm{sEH}^{+/+}$and $\mathrm{sEH}^{-/-}$mice in the present study. These data suggest that the presence or absence of sEH does not have any specific effect on L-NAME-induced attenuation of CRH response in both $\mathrm{sEH}^{+/+}$and $\mathrm{sEH}^{-/-}$mice. Similarly, Zhang et al. described that the inhibition of the activity of sEH enzyme ameliorates endothelial dysfunction in the $d b / d b$ mice, which is NO- 
independent, but dependent on cyp450-epoxygenase-derived metabolites (EETs) (103). Our studies have shown that vascular relaxation through $\mathrm{A}_{2 \mathrm{~A}} \mathrm{AR}$ was independent of $\mathrm{NO}$ and cyclooxygenase pathways $(57,58,60)$. Also, others $(17,39,45)$ reported that $\mathrm{sEH}^{-1-}$ mice were associated with elevated EETs levels compared to $\mathrm{sEH}^{+/+}$mice, and these EETs exerted cardioprotective effects against ischemia/reperfusion injury (77). Therefore, the enhanced CRH in $\mathrm{sEH}^{-/}$compared to $\mathrm{sEH}^{+/+}$mice seems to be independent of the NO pathway, but dependent on the changes associated with sEH-deletion. Although cyclooxygenase (COX) pathway was found to be involved in myocardial reactive hyperemia in dogs, and a cross-talk between COX and nitric oxide pathways in dog coronary vessels was suggested (72), a later publication by Hellmann et al. (27) suggested that COX-metabolites, prostanoids, were not involved in reactive hyperemia in human skin. Accordingly, the role of prostanoids in mouse CRH was not investigated in our study, and therefore, cannot be ruled out.

In addition to the increase in 14,15-EET/DHET ratio in $\mathrm{sEH}^{--}$mice, 13-hydroxylated linoleic acid (13-HODE, hydroxyoctadecadienoic acid) was also increased in $\mathrm{sEH}^{-/-}$versus $\mathrm{sEH}^{+/+}$. 13-HODE may play an anti-inflammatory role and may act as a PPAR $\gamma$-agonist in inflammatory diseases as reported by others $(2,4,18,21,88)$. Also, Guido et al., (15) reported that 13-HODE was involved in canine splenic as well as coronary artery relaxation through prostacyclin $\left(\mathrm{PGI}_{2}\right)$ biosynthesis in both endothelial and smooth muscle cells. Also, in the present study, no change was observed in 9-HODE (hydroxyoctadecadienoic acid), which is considered pro-inflammatory, activating G protein coupled receptor and JNK $(26,66)$ in both $\mathrm{sEH}^{-/}$and $\mathrm{sEH}^{+/+}$mice. Therefore, an increase in 13-HODE in $\mathrm{sEH}^{-/-}$mouse heart perfusate may possibly have a role in enhancing $\mathrm{CRH}$. 
Other linoleic acid metabolites, such as 9,10-,12,13-EpOMEs (epoxyoctadecaenoic acid) and 9,10-,12,13-DiHOMEs (dihydroxyoctadecaenoic acids) were detected in $\mathrm{sEH}^{+/+}$and $\mathrm{sEH}^{-/-}$ mouse heart perfusate in the current study. The increased EpOMEs/DiHOMEs ratios was the result of increased EpOMEs and decreased DiHOMEs in $\mathrm{sEH}^{-/-}$versus $\mathrm{SEH}^{+/+}$mice. In support of our data, Lee et al. (44) reported that EpOMEs/DiHOMEs ratios were increased in response to sEH inhibition by AUDA in vivo, and improved renal recovery against ischemia/reperfusion injury in C57BL/6 mice. Not only that, Nowak et al., (65) reported that primary cultures of rabbit renal proximal tubular cells (RTPC) were protected by the pretreatment of 12,13-EpOME against hypoxia/reoxygenation injury whereas, 12,13-DiHOME did not have a protective effect. Based on our findings, the increase in EpOMEs/DiHOMEs ratio in $\mathrm{sEH}^{-/-}$mouse heart perfusate may possibly play a role in enhancing CRH along with increased 14,15-EET/DHET ratio and 13HODE.

Interestingly, 5-, 11-, 12-, and 15-HETEs (hydroxyeicosatetraenoic acids) data in the current study demonstrate a significant impact after 15-second ischemia. Surprisingly, there was no change in the level of these mid-chain HETEs between $\mathrm{sEH}^{-/-}$and $\mathrm{sEH}^{+/+}$mice. Mid-chain HETEs (5-, 11-, 12-, and 15-HETEs) are arachidonic acid (AA)-derived metabolites produced by allylic oxidation of AA by the action of lipoxygenase (LOX) (39). Increased formation of these mid-chain HETEs is involved in cardiovascular dysfunction through endothelial cells, smooth muscle cells, and monocytes $(13,68,87,97)$. These mid-chain HETEs had direct chemo-toxic effects on vascular tone and on the production of endothelial vascular factors $(29,56,86,93)$. Our data show that 5-, 11-, 12-, and 15-HETEs were significantly decreased after a brief 
ischemia of 15 -seconds in both $\mathrm{sEH}^{-/-}$and $\mathrm{sEH}^{+/+}$mice. In contrast to our findings, $\mathrm{Li}$ et al. reported that the plasma levels of 5-, 9-, 11-, and 15-HETEs were elevated in $\mathrm{sEH}^{-/}$compared to $\mathrm{sEH}^{+/+}$mice (45). This discrepancy could be explained by the different source of the samples; ours were from the isolated heart perfusate, whereas theirs were from the plasma (45). Nevertheless, $\mathrm{CRH}$ was enhanced more in $\mathrm{sEH}^{-/}$compared to $\mathrm{sEH}^{+/+}$mice. Based on the described effects of these mid-chain HETEs, CRH response to brief ischemia in both $\mathrm{sEH}^{-1-}$ and $\mathrm{sEH}^{+/+}$mice may have been enhanced by the decrease in 5-, 11-, 12-, and 15-HETEs. Also, deletion of sEH did not have an effect on the level of these HETEs in the heart perfusate samples.

In summary, the present data suggest that there is a relationship between the increase in coronary reactive hyperemia $(\mathrm{CRH})$ and deletion of soluble epoxide hydrolase $\left(\mathrm{sEH}^{-1}\right)$ in both male and female mice. CRH is mediated, in part, by PPAR $\gamma$ activation in mice, as demonstrated by inhibition of CRH with a PPAR $\gamma$-antagonist and enhanced CRH with a PPAR $\gamma$-agonist. Likewise, CRH is also dependent on nitric oxide (NO), but the enhanced CRH presented by $\mathrm{sEH}^{-1-}$ mice cannot be solely explained through changes in PPAR $\gamma$ or NOS. However, deletion of sEH resulted in significant changes in several oxylipins, such as increased EETs/DHETs ratio, increased EpOMEs/DiHOMEs ratios, and increased 13-HODE. These oxylipins are reported to provide protection in other models of ischemia/reperfusion injury. Thus, our data suggest that these differences in oxylipin metabolism contribute to the enhanced $\mathrm{CRH}$ in $\mathrm{sEH}^{-/-}$mice. Therefore, we conclude that deletion of sEH enhances CRH through increased 14,15-EET/DHET ratio, increased 13-HODE, and increased EpOMEs/DiHOMEs ratio. 


\section{Perspective and Significance}

These findings demonstrate that sEH-deletion is associated with significant changes in arachidonic acid-derived metabolites (EETs), and linoleic acid-derived metabolites (EpOMEs, DiHOMEs, and HODEs). Any dysregulation in these oxylipins signaling pathways or allelic variations may possibly lead to disturbances in regular coronary circulation and may have clinical implications $(42,43)$, such as coronary artery disease in humans with blunted or compromised hyperemic response. Current findings suggest that deletion of sEH, enhances CRH through increased 14,15-EET/DHET ratio, increased 13-HODE, and increased EpOMEs/DiHOMEs ratio. Therefore, targeting sEH through pharmacology could be a feasible option for clinical implementation in cardiovascular diseases in which the hyperemic response is blunted or compromised. 


\title{
CHAPTER TWO
}

\section{Effect of Soluble Epoxide Hydrolase Inhibition on Coronary Reactive Hyperemia: Role of Oxylipins and PPAR $\gamma$}

(This chapter was published in PLoS One journal)

(2016 Sep 1;11(9):e0162147. doi: 10.1371/journal.pone.0162147. eCollection 2016.

\author{
Ahmad Hanif ${ }^{1}$, Matthew L. Edin ${ }^{2}$, Darryl C. Zeldin ${ }^{2}$, Christophe Morisseau ${ }^{3}$, and Mohammed A. \\ Nayeem $^{1}$ \\ ${ }^{1}$ Basic Pharmaceutical Sciences, School of Pharmacy, Center for Basic and Translational Stroke \\ Research. West Virginia University, Morgantown, WV \\ ${ }^{2}$ Division of Intramural Research, NIEHS/NIH, Research Triangle Park, NC \\ ${ }^{3}$ University of California at Davis, One Shields Avenue, Davis, CA
}

\begin{abstract}
:
Coronary reactive hyperemia $(\mathrm{CRH})$ is a physiological response to ischemic insult that prevents the potential harm associated with an interruption of blood supply. The relationship between the pharmacologic inhibition of soluble epoxide hydrolase (sEH) and CRH response to a brief ischemia is not known. sEH is involved in the main catabolic pathway of epoxyeicosatrienoic acids (EETs), which are converted into dihydroxyeicosatrienoic acids (DHETs). EETs protect against ischemia/reperfusion injury and have numerous beneficial physiolgical effects. We hypothesized that inhibition of sEH by $t$-AUCB enhances $\mathrm{CRH}$ in isolated mouse hearts through changing the oxylipin profiles, including an increase in EETs/DHETs ratio. Compared to controls, $t$-AUCB-treated mice had increased CRH, including repayment volume (RV),
\end{abstract}


repayment duration, and repayment/debt ratio $(\mathrm{p} \leq 0.05)$. Treatment with $t$-AUCB significantly changed oxylipin profiles, including an increase in EET/DHET ratio, increase in EpOME/DiHOME ratio, increase in the levels of HODEs, decrease in the levels of mid-chain HETEs, and decrease in prostanoids $(\mathrm{p} \leq 0.05)$. Treatment with MS-PPOH (CYP epoxygenase inhibitor) reduced $\mathrm{CRH}$, including RV ( $\mathrm{p} \leq 0.05)$. Involvement of PPAR $\gamma$ in the modulation of CRH was demonstrated using a PPAR $\gamma$-antagonist (T0070907) and a PPAR $\gamma$-agonist (rosiglitazone). T0070907 reduced CRH ( $\mathrm{p} \leq 0.05)$, whereas, rosiglitazone enhanced CRH ( $\mathrm{p} \leq$ $0.05)$ in isolated mouse hearts compared to the non-treated. These data demonstrate that $\mathrm{sEH}$ inhibition enhances, whereas CYP epoxygenases-inhibition attenuates CRH, PPAR $\gamma$ mediate CRH downstream of the CYP epoxygenases-EET pathway, and the changes in oxylipin profiles associated with sEH-inhibition collectively contributed to the enhanced CRH. 


\section{Introduction}

Cytochrome P450 (CYP) epoxygenases catalyze the epoxidation of arachidonic acid (AA) to form epoxyeicosatrienoic acids (EETs). Produced in endothelial cells, EETs have numerous biological functions, including hyperpolarization and relaxation of vascular smooth muscle cells by activating large conductance $\mathrm{Ca}^{2+}$-activated $\mathrm{K}^{+}$channels $\left(\mathrm{BK}_{\mathrm{Ca}}\right)(9,20)$. EETs are rapidly metabolized through hydration by soluble epoxide hydrolase (sEH) to the less active dihydroxyeicosatrienoic acids (DHETs). Although hydration by $\mathrm{sEH}$ is the main catabolic pathway of EETs (83), two EET isomers, 5,6- and 8,9-EET, are substrates for the cyclooxygenase (COX) pathway (32). Different strategies can increase the levels of EETs to study their effects, including transgenic models $(17,59,77)$ and pharmacologic targeting of sEH (61) or CYP epoxygenases (69). EET generation was increased in CYP2J2-over-expressed mouse myocytes and shown to exert cardioprotective effects against ischemia/reperfusion injury (77). In addition to EETs, targeting sEH impacts the levels of oxylipins (epoxyoctadecaenoic acids [EpOMEs], dihydroxyoctadecaenoic acids [DiHOMEs], hydroxyoctadecadienoic acids [HODEs], hydroxyeicosatetraenoic acids [HETEs], and prostanoids) (45, 47, 75, 76). Lee et al. reported that sEH inhibition by AUDA was associated with an increased EpOME/DiHOME ratio and improved renal recovery against ischemia/reperfusion injury in C57BL/6 mice (44). 13HODE enhanced prostacyclin $\left(\mathrm{PGI}_{2}\right)$ biosynthesis and was involved in splenic and coronary artery relaxation in Mongrel dogs (15). Also, mid-chain HETEs had direct chemo-toxic effects on vascular tone and on the production of endothelial vascular factors $(29,56,86,93)$. Further, studies indicated that EET biological effects could be mediated by peroxisome proliferatoractivated receptor-gamma $(\operatorname{PPAR} \gamma)(8,14,46,70)$. For example, EET-induced aortic relaxation in mice was mediated by $\operatorname{PPAR} \gamma(59,70)$. 
When the heart is subjected to a brief period of ischemia, it responds by momentarily increasing coronary blood flow (12), known as reactive hyperemia (RH) or coronary $\mathrm{RH}(\mathrm{CRH})$. The phenomenon of $\mathrm{CRH}$ is associated with significant coronary vasodilation in response to a cessation in coronary perfusion. Ischemic insult is associated with potentially detrimental effects on cardiac function. Increased blood flow associated with CRH prevents or decreases injury or damage due to ischemia. By supplying an increased amount of blood to the deprived heart muscle, CRH speeds up functional recovery and washes away accumulated metabolic byproducts (105). CRH is considered a protective mechanism, which is blunted or compromised in pathologic conditions affecting the coronary circulation, including cardiac hypertrophy (36), metabolic syndrome (6), unstable angina, myocardial infarction, and congestive heart failure (30). The metabolic mediators involved in RH have been studied extensively, and include adenosine $(5,36,37)$, nitric oxide (NO) $(36), \mathrm{K}_{\text {ATP }}$ channels (36) and hydrogen peroxide $\left(\mathrm{H}_{2} \mathrm{O}_{2}\right)$ $(36,80)$.

The potential effects of pharmacologic inhibition of CYP epoxygenases, inhibition of sEH, and associated changes in oxylipin profiles on CRH in response to a short period of ischemia have not been investigated. We hypothesized that inhibition of sEH enhances CRH through modulation in oxylipin profiles and PPAR $\gamma$, whereas inhibition of CYP epoxygenases attenuates CRH in isolated mouse hearts. 


\section{Materials and Methods}

\section{Animals}

The generation of sEH null $\left(\mathrm{sEH}^{-/-}\right)$mice was described by Sinal et al. (83). $\mathrm{sEH}^{-/-}$and wildtype $\left(\mathrm{WT}, \mathrm{sEH}^{+/+}\right.$) mice were of the $\mathrm{C} 57 \mathrm{BL} / 6$ genetic background and provided by Dr. Darryl Zeldin, National Institute of Environmental Health Sciences/National Institutes of Health (NIH). All animal care and experimentation protocols were submitted to, approved, and carried out in accordance with the West Virginia University Institutional Animal Care and Use Committee and were in accordance with the principles and guidelines of the NIH's Guide for the Care and Use of Laboratory Animals. Both male and female mice (14-16 wks old) in equal ratio were used in our study. Mice were maintained in cages with a 12:12 h light-dark cycle and free access to standard chow and water.

\section{Langendorff-Perfused Heart Preparation}

There are two available Langendorff technique modes to perfuse the heart: 1) constant pressure mode and 2) constant flow mode. In our experiments, we selected the constant pressure mode. Because the two modes have important differences, the selection between the two is particularly important when assessing the role of coronary regulatory mechanisms in response to changing coronary flow (CF) conditions or pathologies, such as ischemia. The constant pressure mode is physiologically more relevant in experiments involving ischemia, like the model in our experiments; therefore, it was selected over the other mode (84).

Soluble epoxide hydrolase null $\left(\mathrm{sEH}^{-/-}\right)$and wild-type (WT; $\left.\mathrm{sEH}^{+/+}\right)$mice $(14-16 \mathrm{wks}$.) of both sexes (equal ratios) were euthanized with sodium pentobarbital $(100 \mathrm{mg} / \mathrm{kg}$ body weight intra- 
peritoneally). Hearts were excised and immediately placed into heparinized $(5 \mathrm{U} / \mathrm{mL})$ ice-cold Krebs-Henseleit buffer containing (in $\mathrm{mM}$ ) $119.0 \mathrm{NaCl}, 11.0$ glucose, $22.0 \mathrm{NaHCO}_{3}, 4.7 \mathrm{KCl}$, $1.2 \mathrm{KH}_{2} \mathrm{PO}_{4}, 1.2 \mathrm{MgSO}_{4}, 2.5 \mathrm{CaCl}_{2}, 2.0$ pyruvate, and 0.5 EDTA. After removal of the lungs and tissue surrounding the heart, the aorta was rapidly cannulated with a 20 -gauge, blunt-ended needle and continuously perfused with $37^{\circ} \mathrm{C}$ buffer continuously bubbled with $\left[95 \% \mathrm{O}_{2}\right]-[5 \%$ $\mathrm{CO}_{2}$ ] at a constant perfusion pressure of $80 \mathrm{mmHg}$. The left atrium was excised, and a waterfilled balloon made of plastic wrap was inserted into the left ventricle through the mitral valve. The balloon was connected to a pressure transducer for continuous measurement of left ventricular developed pressure (LVDP) and heart rate (HR). The heart was then immersed in a water-jacketed perfusate bath $\left(37^{\circ} \mathrm{C}\right)$ and left to beat spontaneously. Left ventricular diastolic pressure was adjusted to $2-5 \mathrm{mmHg}$. A flow transducer was installed above the cannulated aorta for continuous measurement of CF with an ultrasonic flow probe (Transonic Systems, Ithaca, NY). A Power-Lab Chart data acquisition system (AD Instruments, Colorado Springs, CO) was used for data acquisition. Heart function was allowed to stabilize for 30-40 min before initiation of CRH. Only hearts whose CF increased by more than two fold after a 15 -second total occlusion were included in the analysis. This demonstrated that the isolated heart preparation was intact to be included in the experiment. Hearts with persistent arrhythmias or LVDP $<80 \mathrm{mmHg}$ were excluded.

\section{Coronary Reactive Hyperemic Response}

After stabilization for 30-40 minutes, baseline CF, HR, and LVDP were recorded. Hearts were subjected to 15 seconds of total occlusion by closing the valve directly above the cannulated heart to bring forth $\mathrm{CRH}$. After $\mathrm{CF}$ returned to pre-CRH baseline levels, post-CRH baseline $\mathrm{CF}$, 
CF tracing, peak hyperemic flow (PHF), HR, LVDP, repayment volume (RV), and repayment duration $(\mathrm{RD})$ recordings were analyzed for each isolated heart. Investigational drugs were infused into the aortic perfusion line using a microinjection pump (Harvard Apparatus, Holliston, MA) for 15 minutes, after which another $\mathrm{CRH}$ was induced and the same parameters analyzed again. Drugs were infused at a rate equivalent to $1 \%$ of $\mathrm{CF}$. The final concentrations, after standardization of dose $(0.01,0.1,1, \& 10 \mu \mathrm{M})$ response for the various drugs used in this study were $10 \mu \mathrm{M}$ for T0070907 (PPAR $\gamma$-antagonist), rosiglitazone (PPAR $\gamma$-agonist), $t$-AUCB (trans4-[4-(3-adamantan-1-yl-ureido)-cyclohexyloxy]-benzoic acid (a selective sEH-inhibitor, University of California, Davis), and $1 \mu \mathrm{M}$ for MS-PPOH (methylsulfonylpropargyloxyphenylhexanamide, CYP-epoxygenases inhibitor). These concentrations are the same, or less, as used in previous studies: rosiglitazone, $10 \mu \mathrm{M}$; (67), t-AUCB, $10 \mu \mathrm{M}$; (104), MS-PPOH, $1 \mu \mathrm{M}(60)$.

\section{Effect of $t$-AUCB on CRH Response}

After stabilization for 30-40 min, isolated WT mouse hearts were subjected to 15 sec of total occlusion. Recordings of the first CRH (baseline CF, CF tracing, LVDP, HR, RV, PHF, and RD) were analyzed for each heart and averaged. $t$-AUCB was infused at a final concentration of 10 $\mu \mathrm{M}$ and $1 \%$ of $\mathrm{CF}$ rate for $15 \mathrm{~min}$, after which another $\mathrm{CRH}$ was induced and the same parameters (baseline $\mathrm{CF}, \mathrm{CF}$ tracing, LVDP, HR, RV, and RD) were recorded again and analyzed.

\section{Effect of $t$-AUCB on CRH Response in $\mathrm{SEH}^{-/}$Mice:}


To investigate the specificity of the sEH-inhibitor, $t$-AUCB, we evaluated its effect on CRH in $\mathrm{sEH}^{--}$mouse hearts, where the sEH gene is globally deleted. We followed the same protocol described above. $\mathrm{CRH}$ was induced in $\mathrm{sEH}^{-/-}$mice before and after infusing $t$-AUCB for $15 \mathrm{~min}$. The two CRH responses were analyzed.

\section{LC-MS/MS Oxylipin Analysis}

Levels of oxylipins (5,6-, 8,9-, 11,12- and 14,15-EET, 5,6-, 8,9-, 11,12- and 14,15-DHET, 5-, 8-, 9-, 11-, 12- and 15-HETE, 9,10- and 12,13-EpOME, 9,10- and 12,13-DiHOME, 9- and 13HODE, 6-keto prostaglandin- $\mathrm{F}_{1 \alpha}\left[6 \mathrm{~K}-\mathrm{PG}-\mathrm{F}_{1 \alpha}\right], \mathrm{PG}-\mathrm{F}_{2 \alpha}$, thromboxane $\mathrm{B}_{2}\left[\mathrm{TxB}_{2}\right], \mathrm{PGD}_{2}$, and $\mathrm{PGE}_{2}$ ) were determined in pre- and post-CRH heart perfusates of WT and $t$-AUCB-treated WT mice through liquid chromatography, tandem mass spectroscopy (LC-MS/MS) as described previously (41). Heart perfusates were collected after the first $30 \mathrm{~min}$ of stabilization and right after reperfusion for $2.5 \mathrm{~min}$. Hearts were immersed in $5 \mathrm{~mL}$ of warm $\left(37^{\circ} \mathrm{C}\right) \mathrm{Krebs}$-Henseleit buffer with $5 \mu \mathrm{L}$ of $10 \mu \mathrm{M} t$-AUCB to block further EET breakdown by sEH. Heart perfusates were collected two times before ischemia (baseline) and pooled together as one sample and two times after ischemia and pooled together as another sample for LC-MS/MS analysis. Samples were stored at $-80^{\circ} \mathrm{C}$ until processing. Samples were spiked with $30 \mathrm{ng}$ PGE2-d4, 10,11- DiHN, and 10,11-EpHep (Cayman) as internal standards, mixed with 0.1 vol of $1 \%$ acetic acid in $50 \%$ methanol, and extracted by serial passage through Oasis HLB C18 3mL columns (Waters, Milford, MA, USA). Columns were washed twice with $0.1 \%$ acetic acid in 5\% methanol and eluted with methanol into glass tubes containing $6 \mu \mathrm{L}$ of $30 \%$ glycerol in methanol. The methanol was then evaporated under a stream of nitrogen gas, and the dried tubes were frozen and stored at $-80^{\circ} \mathrm{C}$ until analysis. Online liquid chromatography of extracted samples was 
performed with an Agilent 1200 Series capillary HPLC (Agilent Technologies, Santa Clara, CA, USA). Separations were achieved using a Halo C18 column (2.7 mm, $10062.1 \mathrm{~mm}$; MAC-MOD Analytical, Chadds Ford, PA), which was held at $50^{\circ} \mathrm{C}$. Mobile phase A was 85:15:0.1 water: acetonitrile: acetic acid. Mobile phase B was 70:30:0.1 acetonitrile: methanol: acetic acid. Flow rate was $400 \mu \mathrm{L} / \mathrm{min}$; Gradient elution was used. Mobile phase percentage B and flow rate were varied as follows: $20 \% \mathrm{~B}$ at $0 \mathrm{~min}$, ramp from 0 to $5 \mathrm{~min}$ to $40 \% \mathrm{~B}$, ramp from 5 to $7 \mathrm{~min}$ to $55 \%$ B, ramp from 7 to 13 min to $64 \%$ B. From 13 to 19 min the column was flushed with $100 \%$ B at a flow rate of $550 \mu \mathrm{L} / \mathrm{min}$. Samples were solvated in $50 \mu \mathrm{l}$ of $30 \%$ ethanol. The injection volume was $10 \mu \mathrm{L}$. Samples were analyzed in triplicate. Analyses were performed on an MDS Sciex API 3000 equipped with a TurboIonSpray source (Applied Biosystems). Turbo desolvation gas was heated to $425^{\circ} \mathrm{C}$ at a flow rate of $6 \mathrm{~L} / \mathrm{min}$. Negative ion electrospray ionization tandem mass spectrometry with multiple reaction monitoring was used for detection.

\section{Effect of MS-PPOH (CYP epoxygenase inhibitor) on CRH Response}

Isolated WT mice hearts were stabilized for 30-40 min, followed by $15 \mathrm{sec}$ of total occlusion. Recordings of the first CRH (baseline CF, CF tracing, LVDP, HR, RV, PHF and RD) were analyzed for each heart and averaged. MS-PPOH was infused at a final concentration of $1.0 \mu \mathrm{M}$ for 15 minutes, after which the second CRH was induced. CRHs before and after MS-PPOH infusion were analyzed and compared.

\section{Effect of T0070907 (PPAR $\gamma$ antagonist) on CRH Response}

Isolated WT mouse hearts were stabilized for 30-40 min followed by $15 \mathrm{sec}$ of total occlusion. Recordings of the first CRH (baseline CF, CF tracing, LVDP, HR, RV, PHF and RD) were 
analyzed for each heart and averaged for each group, as mentioned previously. T0070907 was infused at a final concentration of $10 \mu \mathrm{M}$ and $1 \%$ of $\mathrm{CF}$ rate for $15 \mathrm{~min}$, after which another CRH was induced and the same parameters (baseline CF, CF tracing, LVDP, HR, RV, and RD) were recorded again and analyzed.

\section{Effect of $t$-AUCB and T0070907 on CRH Response}

In this experiment, we investigated a possible link between the role of PPAR $\gamma$ in CRH and pharmacological inhibition of sEH by $t$-AUCB. We evaluated the effects of selective sEHinhibition on CRH followed by PPAR $\gamma$-antagonist. Baseline CRH was induced in WT mice. $t$ AUCB was infused at a final concentration of $10 \mu \mathrm{M}$ for 15 minutes, after which a second CRH was induced. T0070907 was infused while $t$-AUCB was still being infused, at a final concentration of $10 \mu \mathrm{M}$ for another $15 \mathrm{~min}$, and a third CRH was induced. The three CRH responses were analyzed and compared.

\section{Effect of Rosiglitazone on CRH Response}

After stabilization, WT mouse hearts were subjected to $15 \mathrm{sec}$ of total occlusion. As described above, baseline $\mathrm{CRH}$ was induced in each mouse heart. Rosiglitazone was infused at a final concentration of $10 \mu \mathrm{M}$ for $15 \mathrm{~min}$, followed by another CRH. CRHs before and after rosiglitazone infusion were analyzed and compared.

\section{Statistical and Data Analyses}

Flow debt (baseline flow rate multiplied by occlusion duration) and RV (the integral of hyperemic area above the baseline flow) were calculated using "the integral relative to baseline" 
function in the data pad of Lab-Chart 7.0 software. Since absolute coronary flow rates change proportionally with heart mass, the RV and flow debt are presented as $\mathrm{ml} / \mathrm{g}$ wet heart weight, and baseline and peak flow rate data are presented as $\left(\mathrm{mL} \cdot \mathrm{min}^{-1} \cdot \mathrm{g}\right.$ wet heart weight $\left.{ }^{-1}\right)$. Values are means \pm standard error; $n$ represents the number of animals. For data analysis, two-tailed paired $t$-tests were used for paired data analysis, repeated measures ANOVAs were used for populations measured 3 times, and two-way ANOVAs were used to compare data between groups. Differences were considered statistically significant when $\mathrm{p} \leq 0.05$.

\section{Results}

\section{CRH Response}

\section{Effect of $t$-AUCB on CRH Response in WT Mice}

$t$-AUCB enhanced CRH in WT mice (Fig. 1A). Compared to WT mice, $t$-AUCB-treated WT mice had increased RV (41\%; $6.1 \pm 0.5$ and $8.5 \pm 0.4 \mathrm{~mL} / \mathrm{g}$, respectively; $\mathrm{p}<0.05$, Fig. 1B), increased RD (64\%; $1.6 \pm 0.2$ and $2.7 \pm 0.4$, respectively; Fig. 1C), an increased repayment/debt (R/D) ratio (36\%; $1.5 \pm 0.1$ and $2.1 \pm 0.2$, respectively; $p<0.05$; Fig. 1D), and slightly increased PHF (39.7 \pm 0.7 and $41.2 \pm 1.0 \mathrm{~mL} / \mathrm{min} / \mathrm{g}$ respectively, $\mathrm{p}<0.05 ;$ Fig. 1E). Baseline CF, LVPD, and HR were not different between the two groups $(\mathrm{p}>0.05)$. Time-matched control experiments with WT mouse hearts, employing three consecutive inductions of CRH, showed no

change in the $\mathrm{CRH}$ response and no difference in baseline heart functions, including CF, LVDP, and HR (data not shown).

Effect of $t$-AUCB on CRH Response in $\mathrm{SEH}^{-1-}$ Mice 
Using the same experimental protocol as in the preceding section, $t$-AUCB did not have a significant effect on $\mathrm{CRH}$ in $\mathrm{sEH}^{--}$mice, including RV ( $p>0.05$, Fig. 2A), $\mathrm{R} / \mathrm{D}(\mathrm{p}>0.05$, Fig. 2B), RD (p > 0.05, Fig. 2C), baseline CF, PHF, HR, or LVDP (data not shown).

\section{Oxylipin Analysis of Heart Perfusate before and after $\boldsymbol{t}$-AUCB infusion in WT Mice}

Heart perfusate oxylipin levels were determined by LC-MS/MS. Perfusate samples were collected at baseline after stabilization and right after ischemia in WT and $t$-AUCB-treated WT mice. Out of the four EET regioisomers, only 14,15 -EET, its corresponding metabolite $(14,15$ DHET), and 11,12-DHET were detected. An increasing trend in the level of 14,15-EET in $t$ AUCB-treated WT versus WT mice was observed at baseline and post-ischemia, but was not significant ( $p>0.05$, Fig. 3A). However, sEH-metabolized 14,15-DHET significantly decreased in $t$-AUCB-treated WT versus WT mice at baseline and post-ischemia $(\mathrm{p}<0.0001$, Fig. 3B). As a result, the ratio of 14,15-EET/DHET increased in $t$-AUCB-treated WT versus WT mice at baseline (by 96\%) and post-ischemia (by 173\%; p $\leq 0.05$, Fig. 3C). Our technique also detected 11,12-DHET, which decreased in $t$-AUCB-treated WT versus WT mice at baseline and postischemia ( $\mathrm{p}<0.001$, Fig. 3D). There was no differences in levels of 14,15-EET, 14,15-DHET, or 11,12-DHET pre- and post-ischemia within each group.

Our LC-MS/MS detected 4 mid-chain HETEs (5-, 11-, 12-, and 15-HETE) in WT and $t$-AUCBtreated WT mouse heart perfusates. In WT mice, levels of 5-, 11-, 12-, and 15-HETE decreased post-ischemia (after perfusion was reinstated) compared to baseline, but this was only significant for 5-, 11-, and 15-HETE ( $\mathrm{p} \leq 0.05$, Fig. 4A, B, and D). These mid-chain HETEs had a decreasing trend post-ischemia compared to baseline in $t$-AUCB-treated WT mice, but this trend 
was not significant ( $\mathrm{p}>0.05$, Fig. 4A-D). Treatment with $t$-AUCB decreased HETE levels in WT mice, which was significant for 5-, 11-, and 15-HETE at baseline (p < 0.05, Fig. 4A, B, and D) and 11-HETE post-ischemia (p < 0.05, Fig. 4B).

Linoleic acid (LA) epoxides (9,10- and 12,13-EpOME) levels had an increasing trend at baseline and post-ischemia in $t$-AUCB-treated WT versus WT mice, but was not significant $(\mathrm{p}>0.05$, Fig. 5A). The corresponding 9, 10- and 12, 13-DiHOME levels decreased at baseline and postischemia in $t$-AUCB-treated WT versus WT mice ( $<<0.001$, Fig. 5B). As a result, the EpOME/DiHOME ratio increased in $t$-AUCB-treated WT compared to WT mice at baseline and post-ischemia ( $p<0.0001$, Fig. 5C). The measured EpOMEs, DiHOMEs, and EpOME/DiHOME ratio did not change post-ischemia versus baseline within the same group (p > 0.05, Fig. 5A-C).

Other LA hydroxylated metabolites, 9- and 13-HODE, increased in $t$-AUCB-treated WT versus WT mice at baseline and post-ischemia ( $<$ 0.05, Fig. 6); however, neither HODE level changed post-ischemia versus baseline within the same group $(\mathrm{p}>0.05$, Fig. 6).

The levels of $6 \mathrm{~K}-\mathrm{PG}-\mathrm{F}_{1 \alpha}, \mathrm{PG}-\mathrm{F}_{2 \alpha}, \mathrm{TxB}_{2}, \mathrm{PG}-\mathrm{D}_{2}$, and PG-E $\mathrm{E}_{2}$ was also detected by our LCMS/MS. Treatment of WT mouse hearts with $t$-AUCB at baseline and post-ischemia decreased 6-keto-PG-F $F_{1 \alpha}\left(p<0.05\right.$, Fig. 7A), PG-F $F_{2 \alpha}\left(p<0.05\right.$, Fig. 7B), $\mathrm{TxB}_{2}(\mathrm{p}<0.05$, Fig. 7C), PG-D (p < 0.05, Fig. 7D), and PG-E $E_{2}(p<0.05$, Fig. 7E). Compared to baseline WT levels, postischemia WT levels decreased for TxB $\mathrm{B}_{2}$ and PG- $\mathrm{D}_{2}$, but were significant $(\mathrm{p}<0.05)$ only for TxB $\mathrm{B}_{2}$ (Fig. 7C). 


\section{Effect of MS-PPOH (CYP epoxygenase inhibitor) on CRH Response in WT Mice}

MS-PPOH attenuated CRH in WT mice (Fig. 8A). RV was decreased by $19 \%$ in MS-PPOHtreated WT versus WT mice $(6.6 \pm 0.4$ and $5.3 \pm 0.4 \mathrm{~mL} / \mathrm{g}$ respectively, $\mathrm{p} \leq 0.05$, Fig. 8B). Baseline CF was also decreased by $26 \%$ in MS-PPOH-treated WT versus WT mice $(13.2 \pm 0.4$ and $9.7 \pm 0.6 \mathrm{~mL} / \mathrm{g}$ respectively, $\mathrm{p} \leq 0.05$, Fig. 8E). As a result, the debt volume (calculated as the area under the CF curve during the 15 second ischemia) was decreased and, subsequently, the $\mathrm{R} / \mathrm{D}$ ratio was increased in MS-PPOH-treated WT compared to WT mice $(1.9 \pm 0.1$ and $2.4 \pm 0.2$ respectively, $\mathrm{p} \leq 0.05$, Fig. 8C). PHF was slightly decreased in MS-PPOH-treated WT versus WT mice $(33.6 \pm 0.7$ and $32.4 \pm 0.7 \mathrm{~mL} / \mathrm{min} / \mathrm{g}$ respectively, $\mathrm{p} \leq 0.05$, Fig. 1F). RD (Fig. 8D), HR, and LVPD were not different between the two groups ( $p>0.05)$.

\section{Effect of T0070907 (PPAR $\gamma$ antagonist) on CRH response in WT Mice}

Administering T0070907 to WT mouse hearts attenuated CRH. In T0070907-treated WT versus WT mice, RV was decreased by $32 \%$ (from $6.6 \pm 0.7$ to $4.5 \pm 0.6 \mathrm{~mL} / \mathrm{g}, \mathrm{p}<0.05$, Fig. 9A), RD by $50 \%$ (from $2.1 \pm 0.5$ to $1.1 \pm 0.2 \mathrm{~min}, \mathrm{p}<0.05$, Fig. 9B), and baseline CF by $10 \%$ (from 16.3 \pm 0.7 to $15.1 \pm 1.5 \mathrm{~mL} / \mathrm{min} / \mathrm{g}, \mathrm{p}<0.05$, Fig. 9D). The R/D ratio decreased from $1.7 \pm 0.3$ to $1.2 \pm$ 0.1, but was not significant ( $\mathrm{p}>0.05$, Fig. 9C). LVDP, PHF, and HR were not different between the two groups ( $\mathrm{p}>0.05$, data not shown).

\section{Effect of T0070907on $t$-AUCB-enhanced CRH in WT Mice}

Pharmacologic inhibition of sEH by $t$-AUCB enhanced CRH in WT mice, as mentioned earlier

(Fig. 1). This same experiment was repeated with the PPAR $\gamma$-antagonist T0070907 added to 
study its effect on $t$-AUCB-enhanced CRH. Infusion of $t$-AUCB increased RV in WT mice (from $6.0 \pm 0.7$ to $7.6 \pm 0.5 \mathrm{~mL} / \mathrm{g}, \mathrm{p}<0.05$, Fig. 10A), R/D ratio (from $1.4 \pm 0.1$ to $1.9 \pm 0.2, \mathrm{p}<0.05$

Fig. 10B), and RD (from $1.6 \pm 0.2$ to $3.1 \pm 0.3$, p < 0.05, Fig. 10C). $t$-AUCB-enhanced CRH was attenuated by T0070907. RV in $t$-AUCB-treated WT mice was decreased (from 7.6 \pm 0.5 to $4.3 \pm 0.2 \mathrm{~mL} / \mathrm{g}, \mathrm{p}<0.05$, Fig. 10A), as was the R/D ratio (from $1.9 \pm 0.2$ to $1.1 \pm 0.2, \mathrm{p}<0.05$,

Fig. 10B) and RD (from $3.1 \pm 0.3$ to $1.6 \pm 0.3, p>0.05$, Fig. 10C). Baseline CF, PHF, HR and LVDP were not significantly changed among the 3 groups (WT, [WT $+t$-AUCB], and [WT $+t$ AUCB + T0070907], data not shown).

\section{Effect of rosiglitazone (PPAR $\gamma$ agonist) on CRH Response in WT Mice}

Infusion of rosiglitazone enhanced CRH in WT mice. In rosiglitazone-treated WT versus WT mice, RV increased by $33 \%$ (from $6.1 \pm 0.3$ to $8.1 \pm 0.9 \mathrm{~mL} / \mathrm{g}, \mathrm{p}<0.05$, Fig. 11A), RD by $33 \%$ (from $1.6 \pm 0.1$ to $2.1 \pm 0.3 \mathrm{~min}, \mathrm{p}<0.05$, Fig. 11B), R/D ratio by $31 \%$ (from $1.6 \pm 0.1$ to $1.9 \pm$ $0.2, \mathrm{p}<0.05$, Fig. 11C), and baseline $\mathrm{CF}$ from $15.2 \pm 0.1$ to $17.1 \pm 0.7 \mathrm{~mL} / \mathrm{min} / \mathrm{g}(\mathrm{p}<0.05$, Fig. 11D). PHF, LVDP and HR were not different between the two groups ( $p>0.05$, data not shown). We summarized our observed results into a proposed schematic diagram (Fig. 12). 




Figure 2.1: Comparison of coronary reactive hyperemia (CRH) before (WT) and after ( $t$ AUCB-treated WT) infusion of $t$-AUCB.

(A) tracing depicting coronary flow (CF) changes at baseline (before $\mathrm{CRH}$ ) and after CRH was induced by 15 -second no-flow ischemia in WT (continuous line) and WT $+t$-AUCB (dashed line). Repayment volume (B), repayment duration (C), repayment/debt ratio (D), and peak hyperemic flow (E) increased in $t$-AUCB-treated WT versus WT mice $(\mathrm{p}<0.05) .{ }^{*} \mathrm{p} \leq 0.05$ versus WT. $n=8$. 


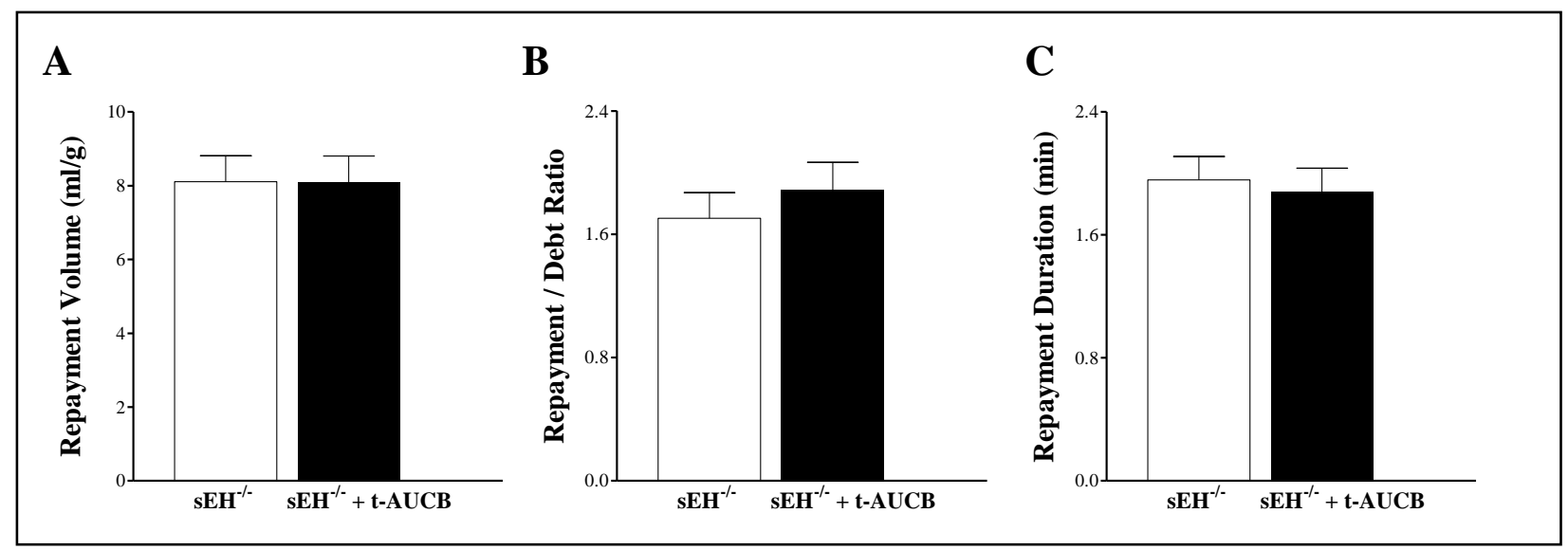

Figure 2.2: Effect of $s E H$-inhibitor, $t$-AUCB, on coronary reactive hyperemia (CRH) in sEH-null $\left(\mathrm{sEH}^{-/}\right)$mice.

$t$-AUCB did not $(\mathrm{p}>0.05)$ affect $\mathrm{CRH}$ in $\mathrm{sEH}^{-/-}$mice, as evident in the unchanged repayment volume (A), repayment/debt ratio $(\mathrm{B})$, and repayment duration $(\mathrm{C})(\mathrm{p}=0.690) . n=6$. 


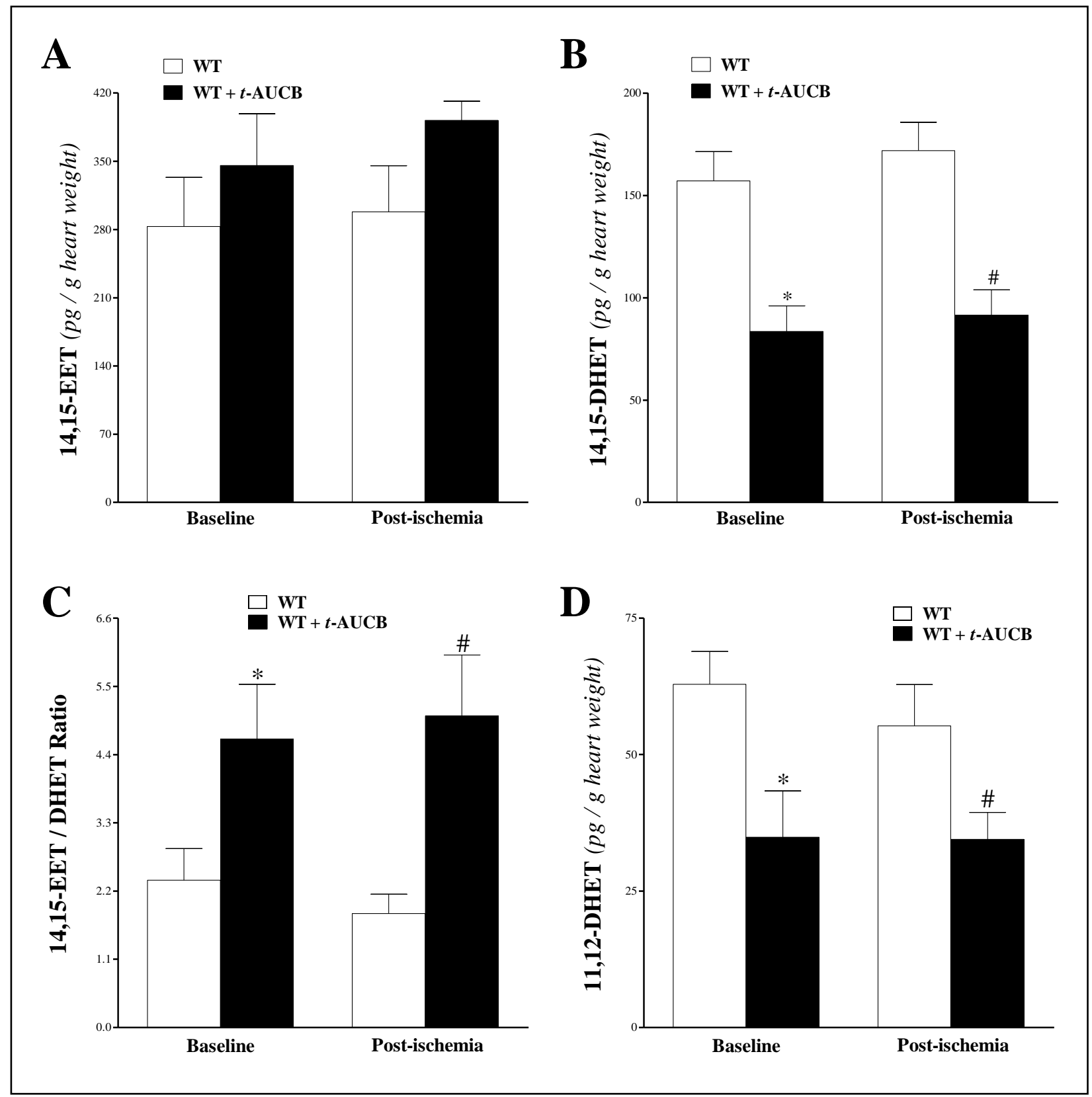

Figure 2.3: LC-MS/MS analysis for 14, 15-EET, 14, 15-DHET, and 11,12-DHET levels in WT and $t$-AUCB-treated WT mouse heart perfusates at baseline (pre-ischemia) and directly after 15-second ischemia (post-ischemia).

(A) At baseline and post-ischemia levels of 14, 15-EET had an increasing trend in $t$-AUCBtreated WT versus WT mice, but this trend was not significant. (B) 14, 15-DHET levels decreased at baseline and post-ischemia in $t$-AUCB-treated WT versus WT ( $<<0.0001)$. (C) The 
14, 15-EET/14, 15-DHET ratio increased $(\mathrm{p}<0.05)$ in $t$-AUCB-treated WT versus WT mice at baseline (by 96\%) and post-ischemia (by 173\%). (D) 11, 12-DHET levels decreased (p < 0.001) at baseline and post-ischemia in $t$-AUCB-treated-WT versus WT mice. There was no difference in 14,15-EET, 14,15-DHET, and 11,12-DHET levels pre- and post-ischemia within each group. $* \mathrm{p} \leq 0.05$ versus baseline WT. $\# \mathrm{p} \leq 0.05$ versus post-ischemia WT. $n=8$.

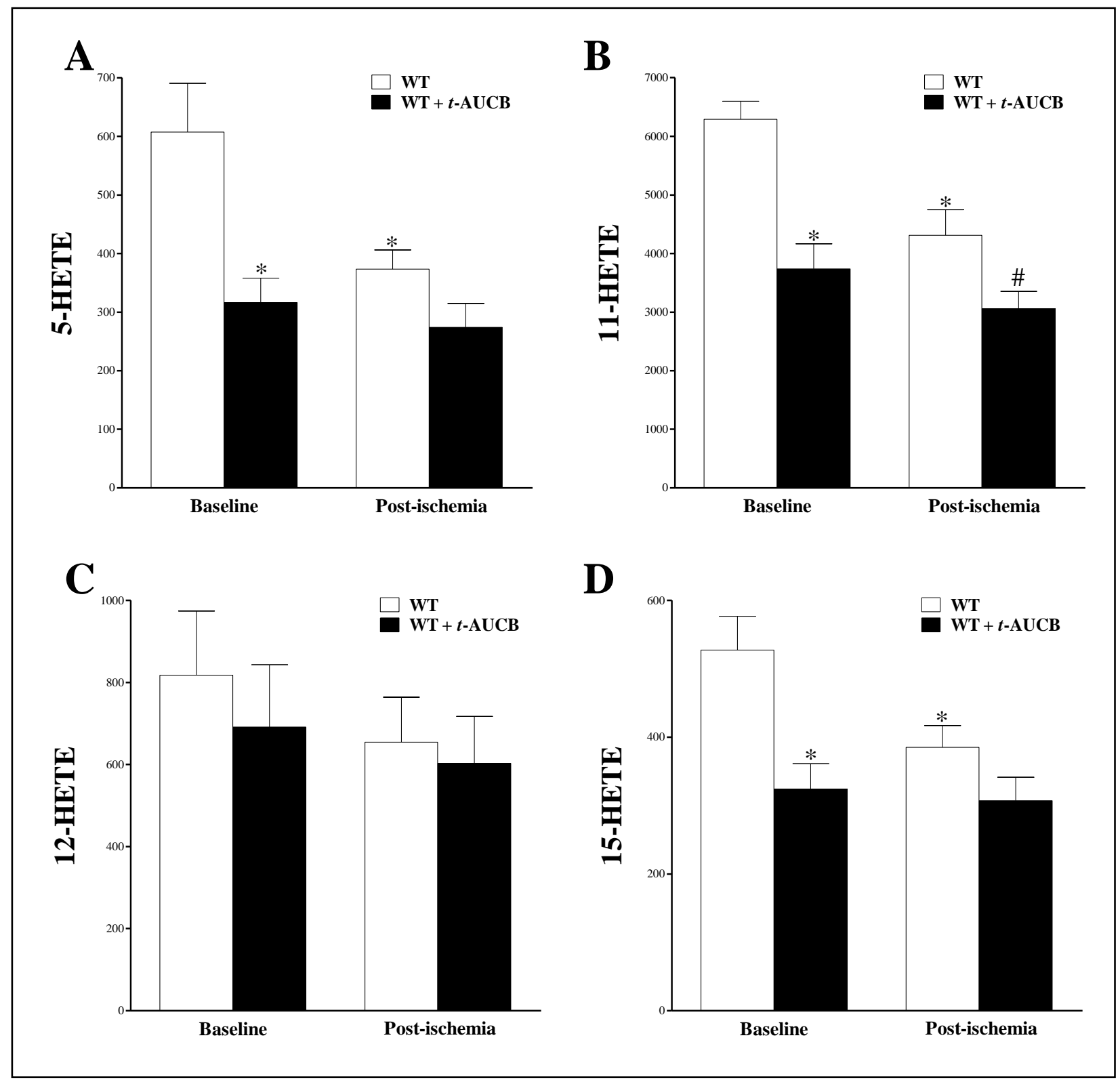


Figure 2.4: LC-MS/MS analysis of 5-, 11-, 12- and 15-HETE levels in WT and $t$-AUCBtreated WT mouse heart perfusates at baseline (pre-ischemia) and post-ischemia.

In WT mice, 5-, 11-, 12- and 15-HETE levels decreased post-ischemia compared to baseline, but this was only significant for 5-HETE (A), 11-HETE (B), and 15-HETE (D) $(\mathrm{p}<0.05)$. The same mid-chain HETEs had a decreasing trend post-ischemia compared to baseline in $t$-AUCB-treated WT mice, but this was not significant ( $\mathrm{p}>0.05$, A-D). Treatment with $t$-AUCB decreased HETE levels in WT mice, which was significant for 5-HETE (A), 11-HETE (B) and 15-HETE (D) levels at baseline $(\mathrm{p}<0.05)$, and in 11-HETE levels post-ischemia $(\mathrm{p}<0.05, \mathrm{~B}) .{ }^{*} \mathrm{p} \leq 0.05$ versus baseline WT. \# $\mathrm{p} \leq 0.05$ versus post-ischemia WT. $n=8$.

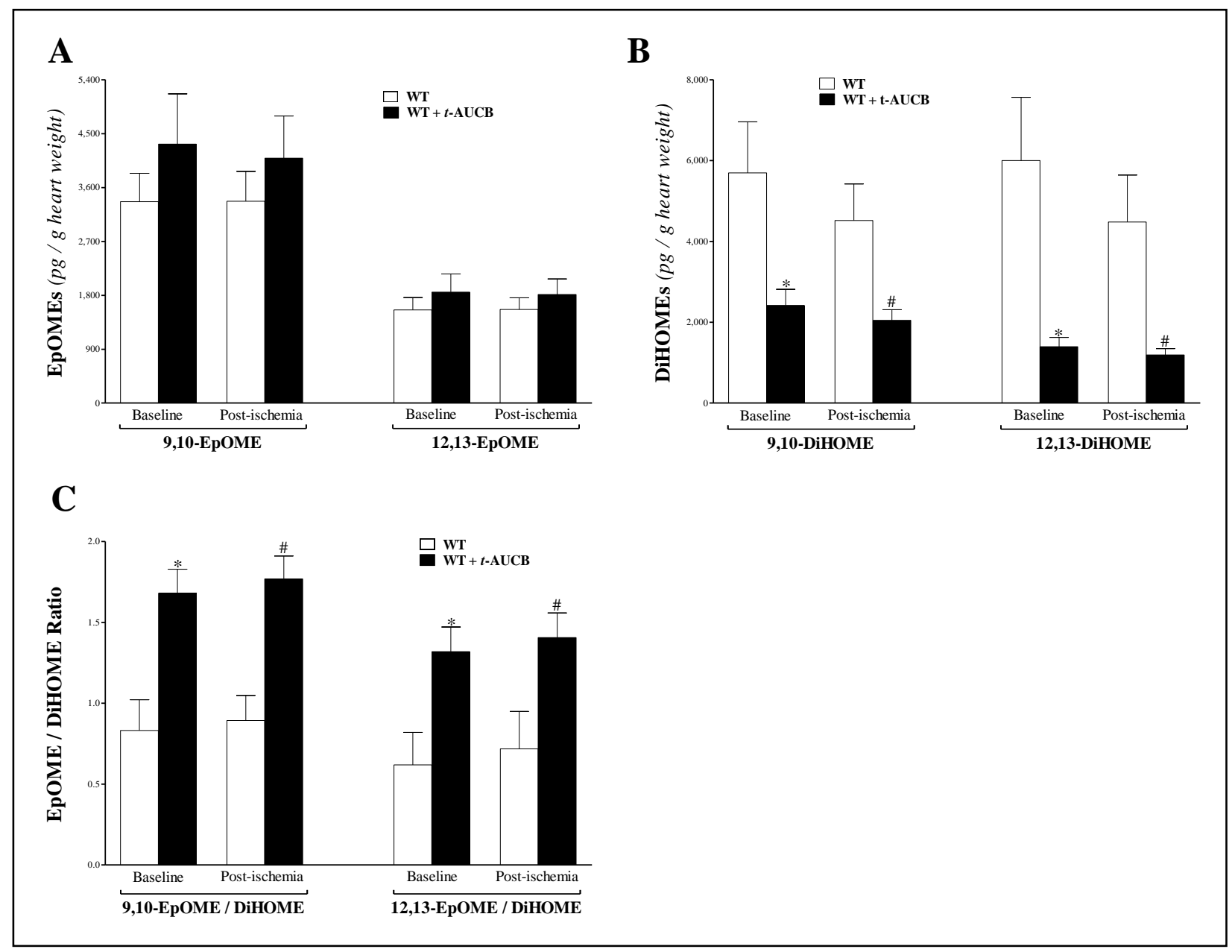


Figure 2.5: LC-MS/MS analysis of EpOME and DiHOME levels and the EpOME/DiHOME ratio in WT and $t$-AUCB-treated-WT mouse heart perfusates at baseline (pre-ischemia) and post-ischemia.

(A) 9,10- and 12,13-EpOME levels had an increasing trend at baseline and post-ischemia in $t$ AUCB-treated-WT versus WT mice, but this was not significant $(\mathrm{p}>0.05)$. Neither EpOME was significantly changed post-ischemia compared to baseline in both groups. (B) 9,10- and 12,13DiHOME levels decreased at baseline and post-ischemia in $t$-AUCB-treated-WT versus WT mice $(\mathrm{p}<0.001)$. (C) The EpOME/DiHOME ratio increased in $t$-AUCB-treated versus WT mice at baseline and post-ischemia $(\mathrm{p}<0.0001)$. The measured EpOME and DiHOME levels and EpOME/DiHOME ratio did not change post ischemia versus baseline within the same group $(\mathrm{p}>$ $0.05, \mathrm{~A}-\mathrm{C}) .{ }^{*} \mathrm{p} \leq 0.05$ versus baseline WT. $\# \mathrm{p} \leq 0.05$ versus post-ischemia WT. $n=8$.

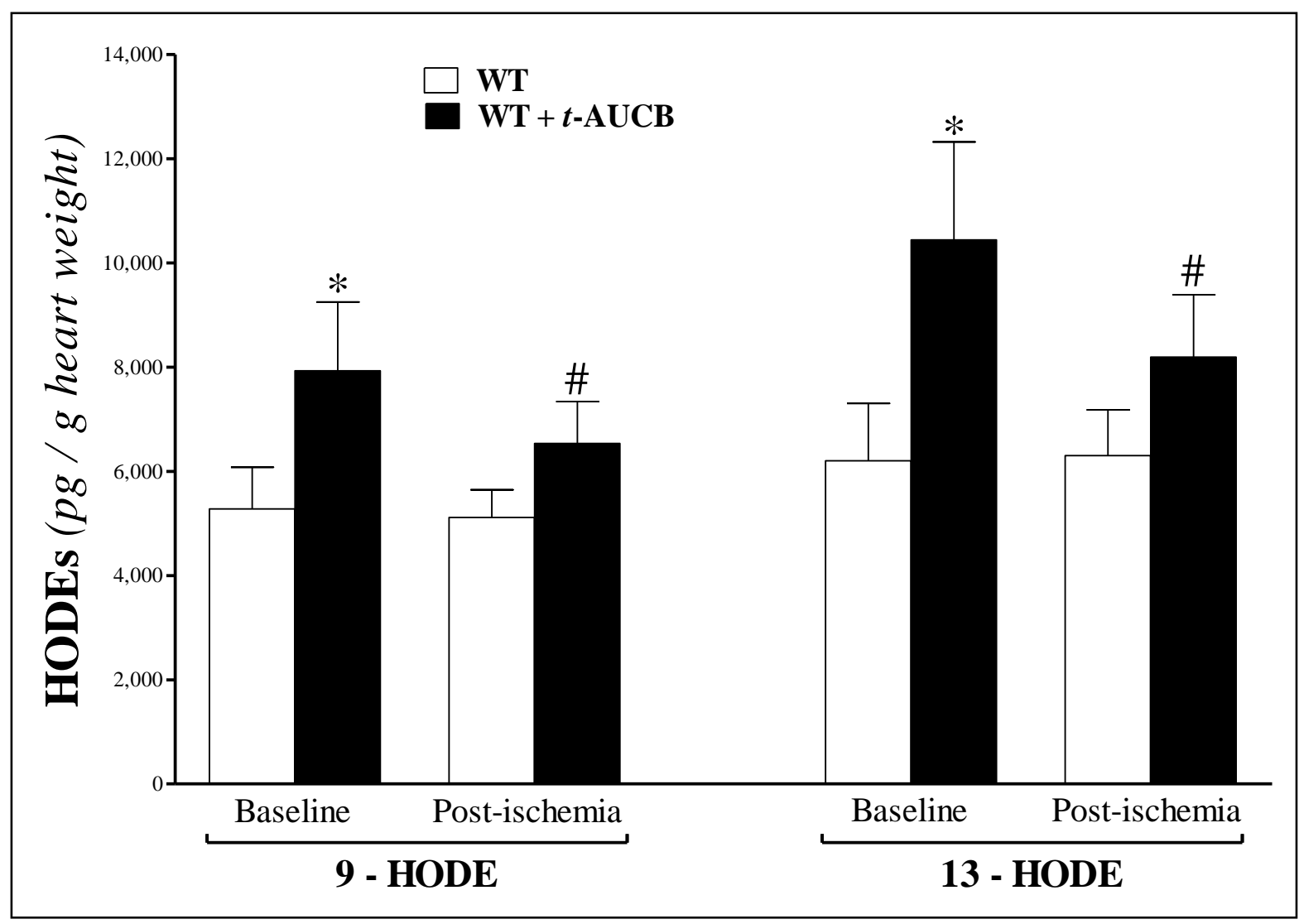


Figure 2.6: LC-MS/MS analysis of HODEs in WT and $t$-AUCB-treated WT mouse heart perfusates at baseline (pre-ischemia) and post-ischemia.

9- and 13-HODE increased in $t$-AUCB-treated WT versus WT mice at baseline and postischemia $(\mathrm{p}<0.05)$. Neither HODE changed post-ischemia versus baseline within the same group $(\mathrm{p}>0.05) .{ }^{*} \mathrm{p} \leq 0.05$ versus baseline WT. $\# \mathrm{p} \leq 0.05$ versus post-ischemia WT. $n=8$.

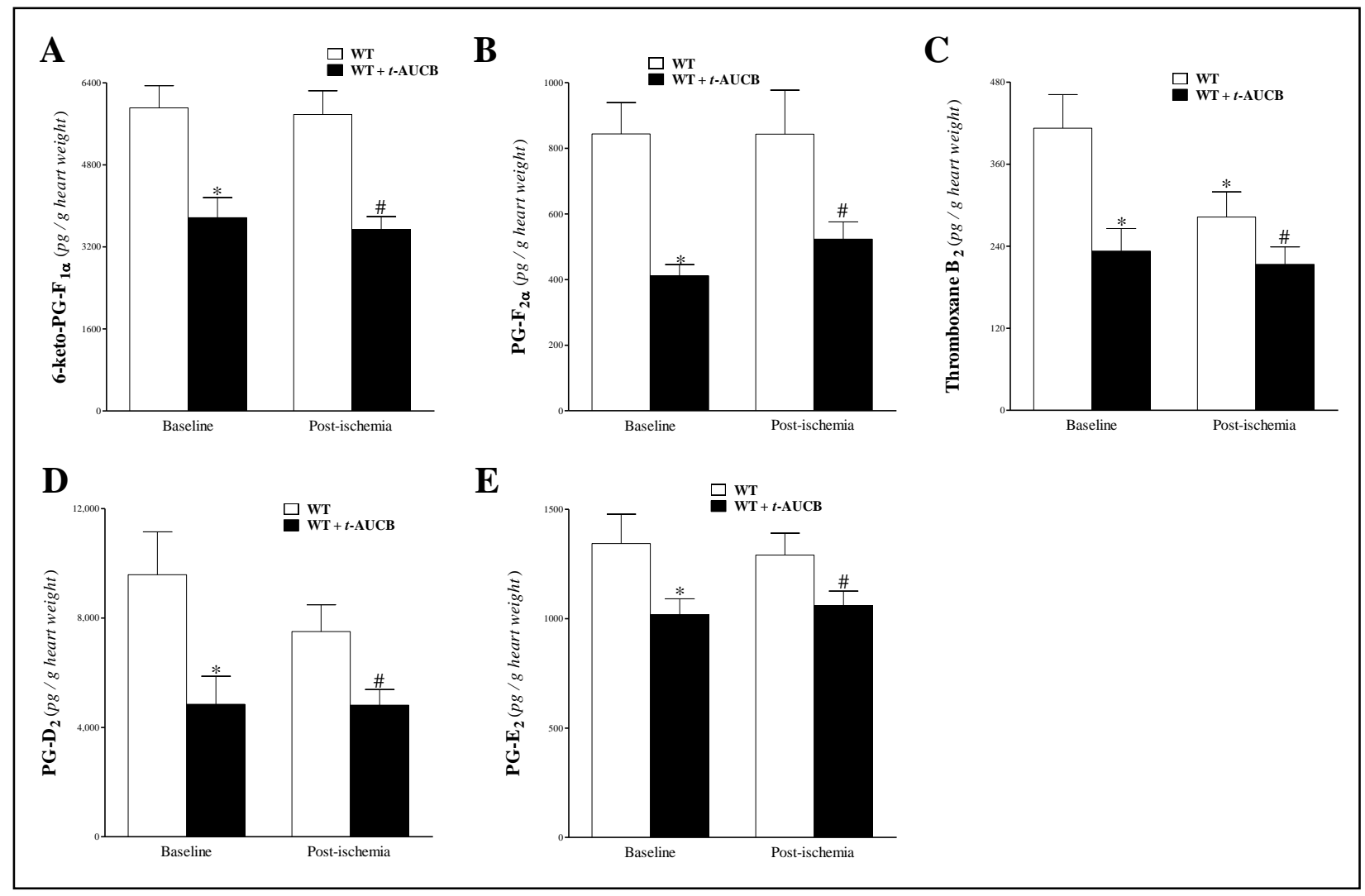

Figure 2.7: LC-MS/MS analysis of 6-keto-PG-F ${ }_{1 \alpha}$, PG-F $_{2 \alpha}$, thromboxane $\mathbf{B}_{2}$, PG- $\mathrm{D}_{2}$, and PG- $E_{2}$ in WT and $t$-AUCB-treated WT mouse heart perfusates at baseline (pre-ischemia) and post-ischemia.

Infusion of $t$-AUCB decreased 6-keto-PG-F $\mathrm{F}_{1 \alpha}(\mathrm{A}), \mathrm{PG}-\mathrm{F}_{2 \alpha},(\mathrm{B})$, thromboxane $\mathrm{B}_{2}(\mathrm{C}), \mathrm{PG}^{-\mathrm{D}_{2}}(\mathrm{D})$, and PG- $\mathrm{E}_{2}(\mathrm{E})$, at baseline and post-ischemia $(\mathrm{p} \leq 0.05)$. Compared to baseline WT, post-ischemia WT levels were decreased for thromboxane $\mathrm{B}_{2}$, and PG- $\mathrm{D}_{2}$, but were significant $(\mathrm{p}<0.05)$ only 
for thromboxane $\mathrm{B}_{2}(\mathrm{C}) .{ }^{*} \mathrm{p} \leq 0.05$ versus baseline WT. $\# \mathrm{p} \leq 0.05$ versus post-ischemia WT. $n=$ 8.

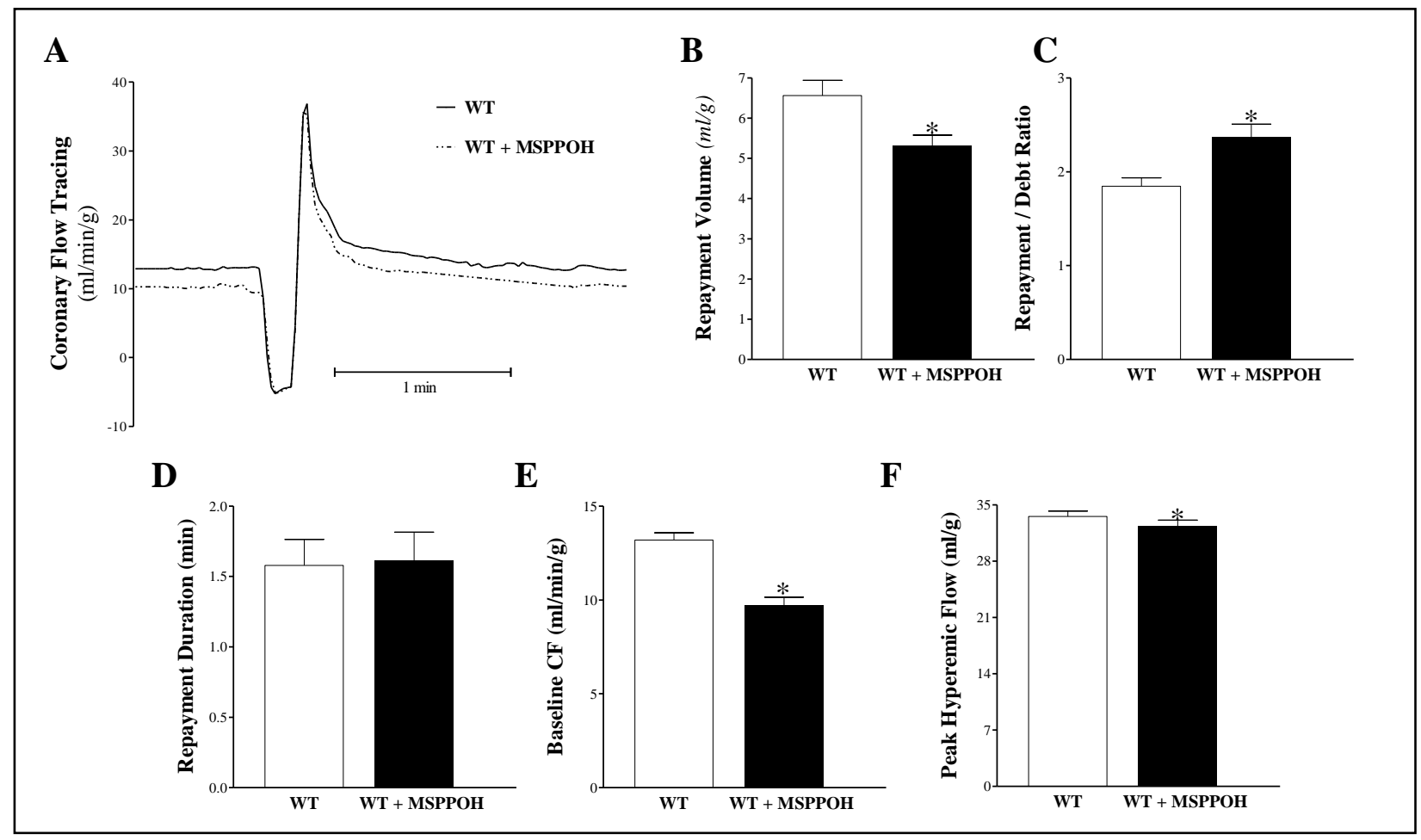

Figure 2.8: Effect of CYP-epoxygenase inhibitor (MS-PPOH, $1 \mu \mathrm{M})$ on coronary reactive hyperemia (CRH) in wild type (WT) mice.

Each WT isolated mouse heart was used as its own control. (A) tracing depicting coronary flow changes at baseline (before $\mathrm{CRH}$ ) and after $\mathrm{CRH}$ was induced by 15 -second no-flow ischemia in WT (continuous line) and MS-PPOH-treated WT (dashed line) mice. Repayment volume (B), baseline CF (E), PHF (F), and LVDP (G) decreased ( $\mathrm{p}$ < 0.05) in MS-PPOH-treated WT versus WT mice. Repayment/debt ratio $(C)$ increased $(\mathrm{p}<0.05)$, whereas repayment duration (D) did not change $(\mathrm{p}>0.05)$ after in MS-PPOH-treated WT versus WT mice. ${ }^{*} \mathrm{p} \leq 0.05$ versus WT. $n=$ 8. 


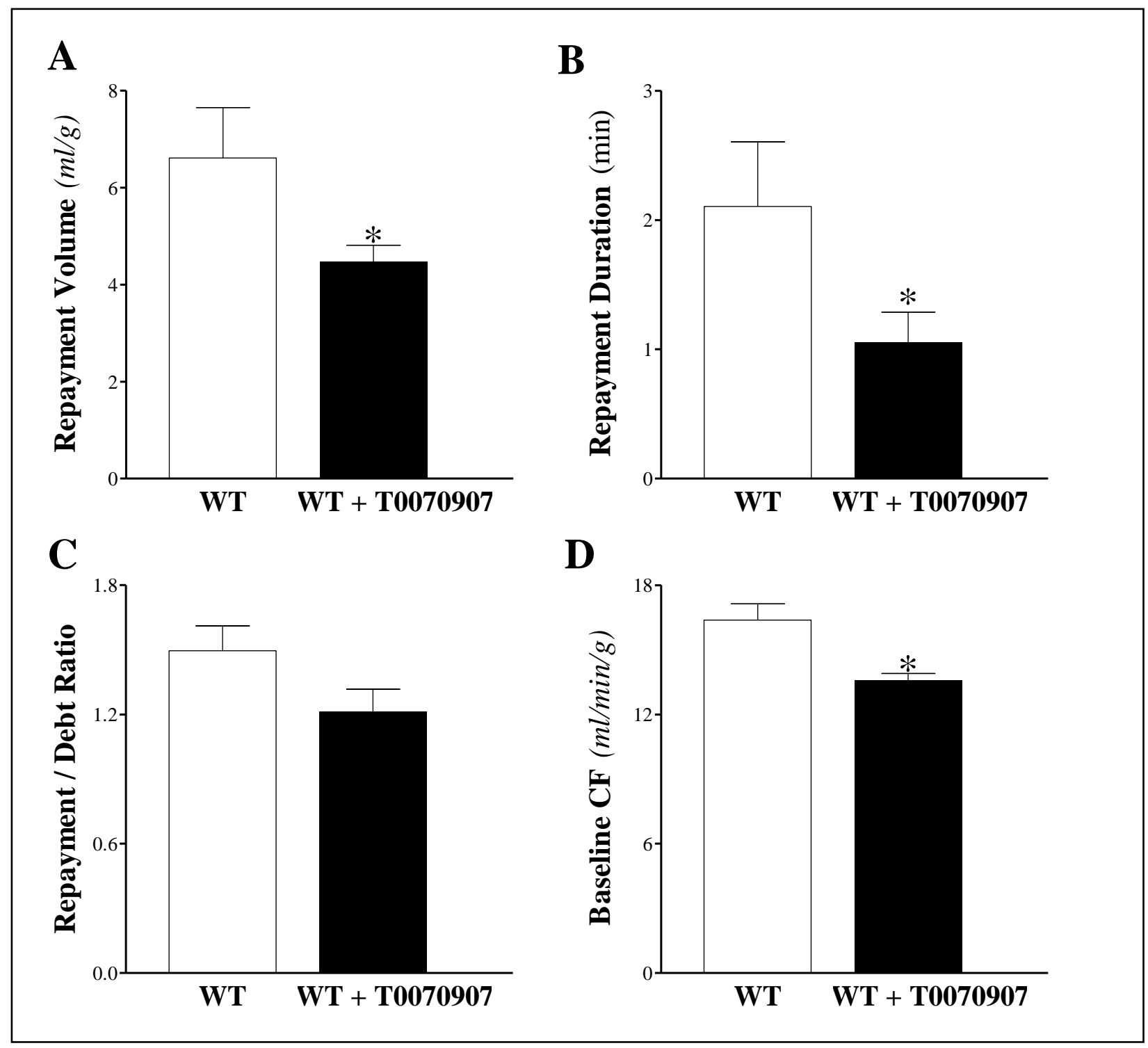

Figure 2.9: Effect of T0070907, PPAR $\gamma$-antagonist $(10 \mu \mathrm{M})$ on coronary reactive hyperemia (CRH) in wild type (WT) mice.

Each WT isolated mouse heart was used as its own control. Infusion of T0070907 into WT mice hearts attenuated CRH. (A) Repayment volume decreased ( $\mathrm{p}<0.05)$, as did RD (B) $(\mathrm{p}<0.05)$ and baseline CF (D) ( $\mathrm{p}<0.05$ ). Repayment/debt ratio (C) had a decreasing trend, but was not significant $(\mathrm{p}>0.05) .{ }^{*} \mathrm{p} \leq 0.05$ versus WT. $n=8$. 


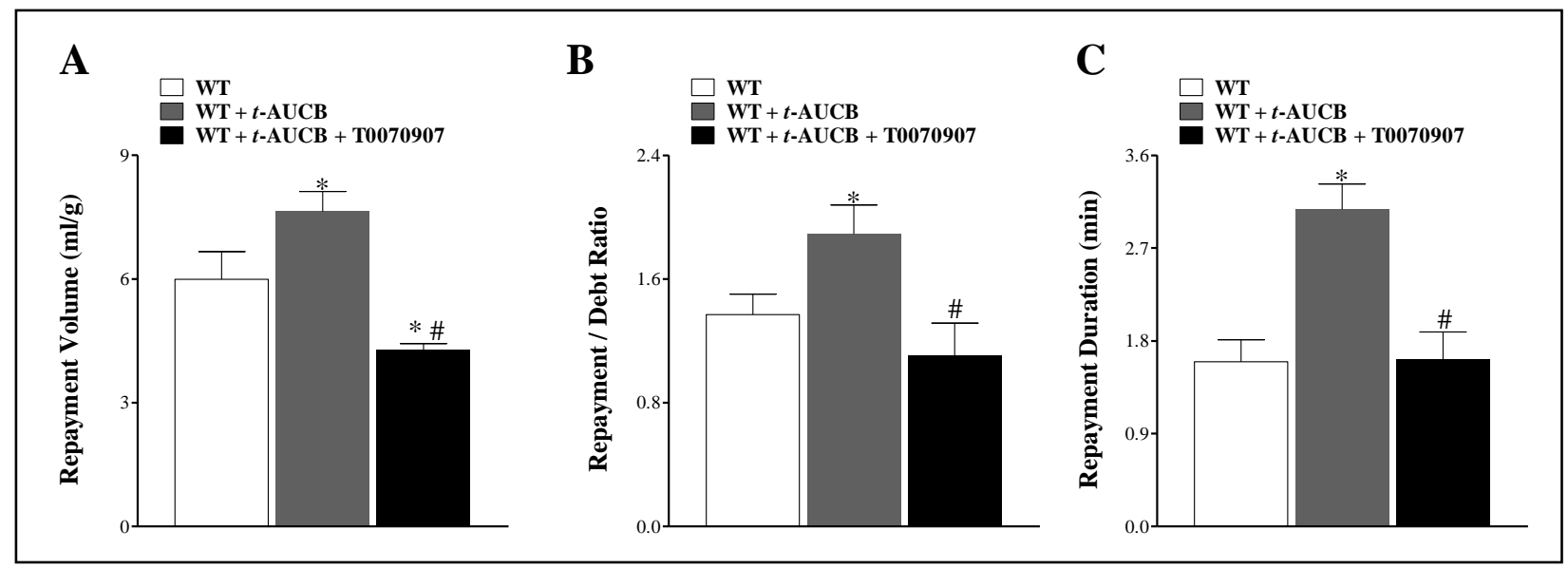

Figure 2.10: Effect of T0070907, PPAR $\gamma$-antagonist $(10 \mu \mathrm{M})$ on $t$-AUCB-enhanced CRH in WT in wild type (WT) mice.

Each WT isolated mouse heart was used as its own control. Inhibition of sEH by $t$-AUCB enhanced CRH in WT mice. Infusion of $t$-AUCB $(10 \mu \mathrm{M})$ increased repayment volume $(\mathrm{RV})(\mathrm{p}$ $<0.05, \mathrm{~A})$, repayment/debt ratio $(\mathrm{p}<0.05, \mathrm{~B})$, and repayment duration $(\mathrm{RD})$ in WT mice $(\mathrm{p}<$ 0.05, C). The $t$-AUCB-enhanced CRH was attenuated by T0070907. T0070907 decreased RV (A), R/D ratio (B), and $\mathrm{RD}(\mathrm{C})$ in $t$-AUCB-treated-WT mice $(\mathrm{p}<0.05) .{ }^{*} \mathrm{p} \leq 0.05$ versus WT. $n=$ 6. 


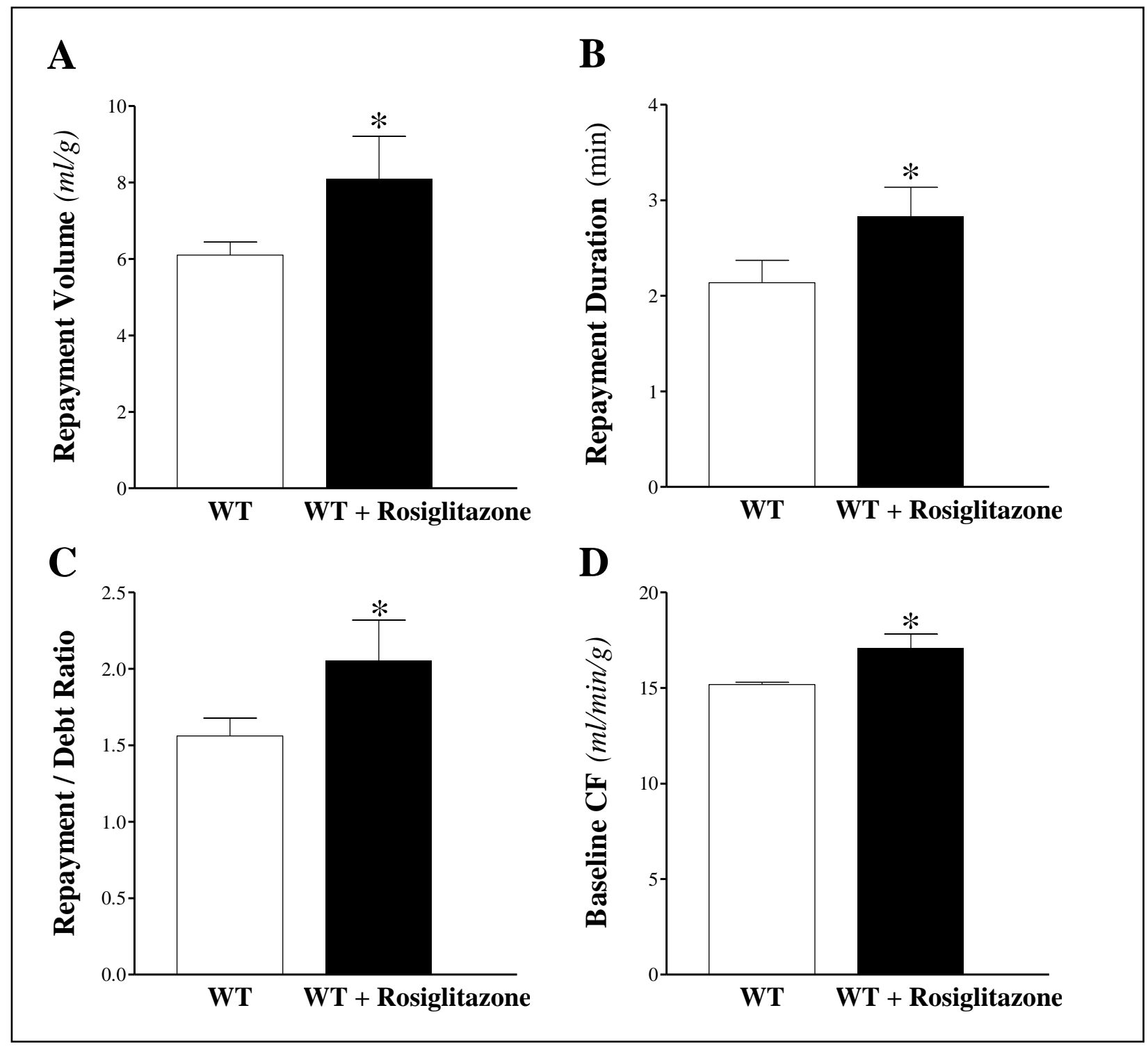

Figure 2.11: Comparison of CRH in WT before and after infusion of rosiglitazone (PPAR $\gamma$ agonist, $10 \mu \mathrm{M})$.

Each isolated heart was used as its own control. Repayment volume (A), repayment duration (B), repayment/debt ratio (C), and baseline $\mathrm{CF}(\mathrm{D})$ increased after rosiglitazone administration (p < $0.05) .{ }^{*} \mathrm{p} \leq 0.05$ versus WT. $n=8$. 

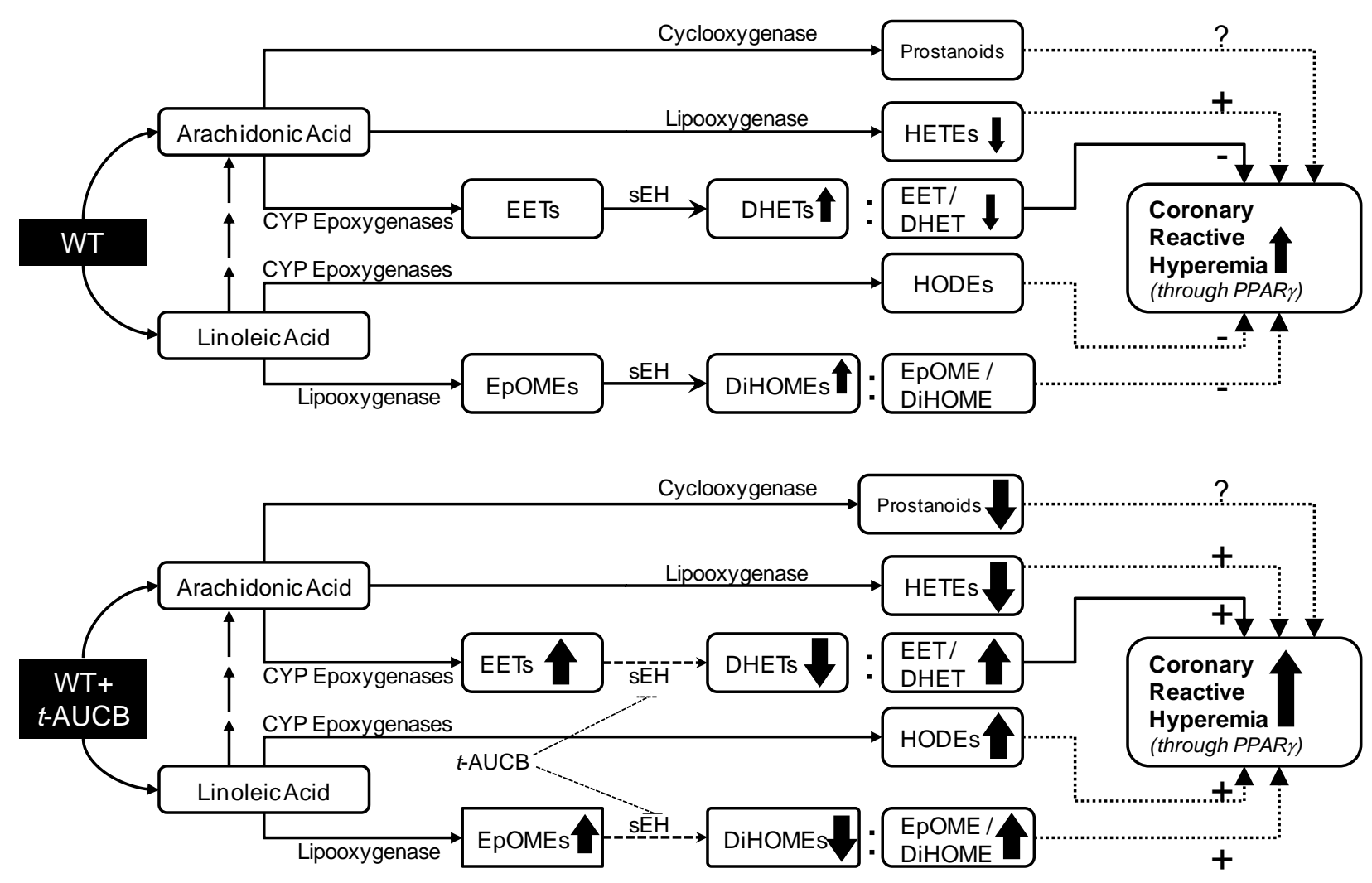

Figure 2.12: A schematic diagram comparing the oxylipin changes observed in response to a brief ischemia and their possible impact on coronary reactive hyperemia (CRH) between WT and $t$-AUCB-treated WT mice.

Treatment with $t$-AUCB enhanced CRH possibly through increased EET/DHET ratio, increased 13-HODE, increased EpOME/DiHOME ratio, decreased mid-chain HETEs, and PPAR $\gamma$ activation. 


\section{Discussion}

Pharmacologic inhibition of sEH in WT mice enhanced CRH in an isolated heart model after brief ischemia. sEH inhibition was also associated with changes in various oxylipin profiles. The relationship among inhibition of CYP epoxygenases, inhibition of sEH, oxylipins, and PPAR $\gamma$ in the modulation of $\mathrm{CRH}$ in isolated mouse hearts is not known. Therefore, this study was designed to investigate the role of sEH, oxylipins, and PPAR $\gamma$ in the modulation of $\mathrm{CRH}$ using isolated WT mouse hearts. Our data demonstrated that: 1) Inhibition of sEH by $t$-AUCB enhanced CRH; 2) Treatment with $t$-AUCB significantly changed oxylipin profiles, including an increased EET/DHET ratio, increased EpOME/DiHOME ratio, increased HODEs, decreased mid-chain HETEs, and decreased prostanoids; 3) Inhibition of CYP epoxygenases (by MSPPOH) attenuated CRH; 4) The PPAR $\gamma$ antagonist T0070907 decreased CRH and $t$-AUCBenhanced CRH, and 5) the PPAR $\gamma$ agonist rosiglitazone increased $\mathrm{CRH}$.

Inhibition of sEH by $t$-AUCB significantly enhanced CRH after brief ischemia compared to non-treated WT mice. The association between compromised CRH and some cardiovascular pathologies $(6,30,36)$ confirms the significance of CRH. Further, the $t$-AUCB-enhanced CRH was accompanied by a 1.0 - (at baseline) to 1.7 - (post-ischemia) fold increase in the 14,15 EET/DHET ratio, primarily driven by a decrease in 14,15-DHET. Similarly, another dihdroxyEET metabolite $(11,12$-DHET) decreased in $t$-AUCB-treated mouse hearts, further confirming the efficacy of the sEH-inhibitor, $t$-AUCB. The specificity of $t$-AUCB as a sEH inhibitor was tested using sEH knockout $\left(\mathrm{sEH}^{--}\right)$mice, where no difference in $\mathrm{CRH}$ between the $t$-AUCBtreated $\mathrm{sEH}^{-/-}$versus non-treated $\mathrm{sEH}^{-/-}$mice was observed. The rapid hydration of EETs (24) may explain the challenge of detecting them. We were able to detect 14,15- 
EET and two diols (11,12- and 14,15-DHETs) in heart perfusate samples. As mentioned earlier, EETs are formed by the epoxidation of arachidonic acid (AA) by CYP epoxygenases and possess well-established beneficial cardiovascular effects $(19,53,59,85$, 102), including protection against ischemia/reperfusion injury (77), and vasodilation in many vascular beds such as the intestines (71), kidney preglomerular vasculature (33), and brain (23). Inhibition of sEH, the main catabolic enzyme of EETs, has been widely used to increase the level of EETs and to investigate their effects (45). Acute inhibition of sEH, through injection of a sEH inhibitor, lowered blood pressure in spontaneously hypertensive rats (SHR) (101), whereas chronic inhibition of sEH lowered angiotensin-II-induced hypertension (34). Besides lowering blood pressure, sEH inhibition ameliorated renal damage in angiotensin-dependent, salt-sensitive hypertension rats (35). In the current study, the brief ischemia had no effect on EET or DHET levels. This could be due to the short duration of ischemia (15 seconds). In contrast, DHETs (EETs metabolites) were elevated in the mouse heart perfusate in response to longer (20-min) ischemia (17). Another interesting finding in the current study was that no significant difference was found in $\mathrm{CRH}$ between male and female mice in either $t$-AUCBtreated or non-treated groups. Based on our data, the enhancement of $\mathrm{CRH}$ associated with sEH inhibition in WT mice may be partially due to an increase in the EET/DHET ratio.

Mid-chain (5-, 11-, 12- and 15-) HETE levels decreased in response to ischemia and to $t$ AUCB treatment at baseline and post-ischemia. Mid-chain HETEs are produced through allylic oxidation of AA by lipoxygenase (LOX) (39). They were shown to have chemotaxis effects, change vascular tone, and induce the production of vascular endothelial growth factors $(29,56$, 86, 93). Also, the increased formation of mid-chain HETEs was involved in cardiovascular dysfunction $(13,68,87,97)$. Unlike the vasodilatory effect of EETs in the kidneys (33), 12- 
HETE caused vasoconstriction in small renal arteries (48). Also, the generation of mid-chain HETEs is increased in essential hypertension (16) suggesting that they could be involved in its pathogenesis. These reports point to opposite effects of EETs and HETEs in vascular biology. Maayah et al. reported that mid-chain HETEs blocked the synthesis of EETs and increased their conversion to DHETs in RL-14 cells (49). Moreover, although sEH is not directly involved in the generation or breakdown of mid-chain HETEs, sEH was found to be essential for mid-chain HETE-mediated induction of cellular hypertrophy (49). Therefore, not only do EETs and midchain HETEs have opposite effects, they seem to affect the level of each other. In contrast to our findings, Li et al. reported that the plasma levels of 5-, 11-, 12-, and 15-HETE were not changed by sEH-inhibition in WT mice (45). However, their results were based on plasma samples, whereas ours were based on isolated heart perfusate samples and demonstrated significant decrease in the level of the same HETEs in response to sEH inhibition. Based on these results, the decrease in mid-chain HETEs in both $t$-AUCB-treated and non-treated mice in response to ischemia suggests that these metabolites are down regulated in response to ischemic episodes. This is an interesting finding because HETEs were the only oxylipins analyzed in this paper whose levels were affected by the brief ischemia. Also, the further decrease of these HETEs by $t$ AUCB-treatment may have played a role in the enhanced CRH observed in $t$-AUCB-treated hearts.

Linoleic acid (LA) epoxidation metabolites, such as 9,10-, and 12,13-EpOMEs, and their corresponding sEH-metabolized DiHOMEs were detected in the heart perfusate samples. As expected, inhibition of sEH by $t$-AUCB was further confirmed by an increased EpOME/DiHOME ratio. This resulted from increased 9,10-,12,13-EpOMEs (albeit not statistically significant), and decreased 9,10-, 12,13-DiHOMEs in $t$-AUCB-treated versus non- 
treated mice. The physiological significance of EpOMEs and DiHOMEs remains poorly understood, and the evidence is somewhat contradictory, with a few studies suggesting toxic effects, while others indicating beneficial effects of these bioactive metabolites. EpOMEs and DiHOMEs were reported to increase oxidative stress in vascular endothelial cells (96); DiHOMEs were toxic to renal proximal tubular cells (54); and intravenously injected 9,10EpOME had cardiodepressive effects in dogs (90). The main caveat of these studies, which reported that EpOMEs and DiHOMEs have toxic effects, is that very high concentrations (100 $500 \mu \mathrm{M})$ were used (39). Contrary to these reports, lower, more physiological concentrations of EpOMEs and DiHOMEs did not have toxic effects and rather had beneficial effects (52). Mitchell et al reported that LA and its oxidative metabolites (EpOMEs and DiHOMEs) did not have toxic effects during acute exposure in Langendorff-perfused rat hearts (52). Pretreatment with 12,13-EpOME protected primary cultures of rabbit renal proximal tubular cells against hypoxia/reoxygenation injury (65). Also, increased EpOME/DiHOME ratio, induced by sEH inhibition using AUDA, improved renal recovery in response to ischemia/reperfusion injury in C57BL/6 mice (44). Based on these findings and on the described biologic role of LA epoxides, the increase in EpOME/DiHOME ratio in $t$-AUCB-treated mouse heart perfusates may have contributed to enhancing $\mathrm{CRH}$.

Linoleic acid (LA) is also metabolized through hydroxylation by CYP epoxygenases to form hydroxyl-LA metabolites known as hydroxyoctadecadienoic acids (HODEs) (39). The detected two HODE isomers, 9-, and 13-HODE, were increased in $t$-AUCB-treated WT. Like EpOMEs, the physiologic functions of HODEs are still being investigated (39). 13-HODE is suggested to have an anti-inflammatory role in inflammatory diseases through its effect as a PPAR $\gamma$-agonist $(2,4,18,21,88)$. Also, 13-HODE, through increasing prostacyclin $\left(\mathrm{PGI}_{2}\right)$ 
biosynthesis, was involved in splenic and coronary artery relaxation in smooth muscle cells in Mongrel dogs (15). 9-HODE, unlike 13-HODE, was described as pro-inflammatory in an experimental wound-healing model in rats $(26,66)$. In contrast to $13-\mathrm{HODE}$, which can be produced from LA by the action of 12/15-LOX, 9-HODE production by LOX has not been reported in humans; rather, mouse 8-LOX metabolizes LA to 9-HODE (66). In contrast to our data, Luria et al. reported no change in 9- and 13-HODE in $\mathrm{sEH}^{-/}$compared to $\mathrm{sEH}^{+/+}$(47). This discrepancy may be explained by the difference in the source of the metabolites: we used heart perfusate samples, whereas Luria et al (47) used urinary samples. Also, the reported metabolic and functional effects of $\mathrm{sEH}^{--}$(genetic deletion of $\mathrm{sEH}$ ) and pharmacologic inhibition of sEH are not necessarily the same (45). Therefore, and based on our results, the increase in 13-HODE in $t$-AUCB-treated mouse heart perfusates might have played a role in enhancing $\mathrm{CRH}$, whereas the role of 9-HODE is not yet known based on its, thus far, described biologic functions.

Treatment of WT mice hearts with $t$-AUCB significantly decreased prostanoid levels, including PGs and $\mathrm{TxB}_{2}$. $\mathrm{PG}_{-} \mathrm{G}_{2}$ and $\mathrm{PG}-\mathrm{H}_{2}, \mathrm{AA}$ metabolites formed by COX isoforms (1 and 2), are converted to the 4 main bioactive PGs $\left(\mathrm{PG}-\mathrm{D}_{2}, \mathrm{PG}-\mathrm{E}_{2}, \mathrm{PG}-\mathrm{I}_{2}, \mathrm{PG}-\mathrm{F}_{2 \alpha}\right)$ and thromboxanes $\left(\mathrm{TxA}_{2}\right.$ and $\left.\mathrm{TxB}_{2}\right)(74,92)$. Most PGs have pro-inflammatory effects. For example, PG-E augments arterial dilation and increases microvascular permeability (92). However, PG-E $\mathrm{E}_{2}$ was found to have an anti-inflammatory role as well by up-regulating cAMP and inducing secretion of the anti-inflammatory IL-10 (78). Similarly, PG- $\mathrm{D}_{2}$ attenuated inflammation in experimental models of pleuritis and colitis (92). 6-keto-PG-F $\mathrm{F}_{1 \alpha}$, the non-active hydrolysis product of prostacyclin $\left(\mathrm{PG}-\mathrm{I}_{2}\right)$, and $\mathrm{TxB}_{2}$, the inactive degradation product of $\mathrm{TxA}_{2}(92)$, were also decreased by $t$-AUCB. PG-F $\mathrm{F}_{2 \alpha}$ is elevated in patients with chronic inflammatory diseases (92). In 
support of our findings, sEH-inhibition by AUDA-BE reversed lipopolysaccharide (LPS)-induced increase in PG- $\mathrm{D}_{2}$, PG-E $\mathrm{E}_{2}$, 6-keto-PG-F $\mathrm{F}_{1 \alpha}$, and $\mathrm{TxB}_{2}$ (76). Also, sEH-inhibition by AUDA-BE, through increasing the concentrations of EETs, inhibited NF- $\kappa B$ translocation (75). Combination of sEH-inhibition by AUDA-BE and COX-inhibition had an additive effect and further decreased PG- $\mathrm{D}_{2}$ and PG-E $\mathrm{E}_{2}$ (75). Therefore, the associated decrease in the measured PGs could be attributed to sEH-inhibition by $t$-AUCB. However, the impact of these changes on the $t$-AUCBenhanced CRH is not yet clear.

As mentioned earlier, EETs are generated from AA by cytochrome P450 (CYP) epoxygenase enzymes, primarily CYP2C and CYP2J. We targeted the CYP epoxygenase-EET pathway using pharmacologic inhibition of CYP-epoxygenases to further support our findings of the involvement of CYP-epoxygenases in modulating CRH. sEH-inhibition increases the level of EETs by blocking their breakdown; we investigated the effect of blocking the synthesis of EETs on CRH using a CYP epoxygenases-inhibitor. Evidently, inhibiting CYP epoxygenases would negatively impact EET synthesis and, therefore, their effects. P450 enzymes are highest in coronary arteries and in small arterioles (20). MS-PPOH was used as an EET-synthesis inhibitor that blocked the role of EETs as mediators of insulin-mediated augmentation of skeletal muscle perfusion (82). Our data indicate that MS-PPOH significantly decreased CRH after a brief ischemia. These findings suggest that inhibition of CYP epoxygenases by MS-PPOH decreased CRH possibly due to inhibition of EETs synthesis. Further studies to investigate the impact of MS-PPOH on oxylipin profiles are needed to understand the metabolic changes associated with the inhibition of CYP epoxygenases. 
This study also showed that CRH was decreased by T0070907, a PPAR $\gamma$-antagonist, whereas rosiglitazone, a PPAR $\gamma$-agonist, increased CRH. Moreover, T0070907 attenuated $t$ AUCB-enhanced CRH in WT mouse hearts. Studies indicated that EET effects could be mediated by PPAR $\gamma$ receptors $(8,14,46,70)$. Liu et al. suggested that selective sEH inhibition potentiates the anti-inflammatory effect in endothelial cells by increasing the retention of EETs so that PPAR $\gamma$ activation is prolonged (46). Our results suggest that PPAR $\gamma$ receptors are involved in modulating $\mathrm{CRH}$. Also, the attenuated $t$-AUCB-enhanced $\mathrm{CRH}$ in WT mice (by T0070907) suggests that PPAR $\gamma$ may mediate CRH downstream of CYP epoxygenases-EET pathway.

In summary, the findings of this study suggest that the CYP epoxygenases-EET pathway may have a role in mediating $\mathrm{CRH}$ possibly through PPAR $\gamma$ activation, as noted by sEHinhibition, which enhanced CRH, and CYP epoxygenases-inhibition, which decreased CRH. Also, sEH-inhibition is accompanied by changes in AA oxylipins (increased EET/DHET ratio, decreased mid-chain HETEs, and decreased prostanoids) and LA oxylipins (increased EpOME/DiHOME ratio, and increased HODEs), which might have collectively accounted for the observed enhancement of $\mathrm{CRH}$. Therefore, we conclude that sEH-inhibition enhances, whereas CYP epoxygenases-inhibition attenuates CRH, PPAR $\gamma$ activation mediates CRH downstream of the CYP epoxygenases-EET pathway, and the changes in oxylipin profiles associated with sEH-inhibition collectively account for the enhanced CRH. 


\section{CHAPTER THREE}

\section{DISCUSSION, SUMMARY and CONCLUSIONS}

\section{Discussion}

Targeting sEH, through pharmacologic inhibition or genetic deletion, enhanced $\mathrm{CRH}$ in an isolated heart model after brief ischemia. sEH disruption was also associated with changes in various oxylipin profiles. We investigated the role of $\mathrm{sEH}$, oxylipins, and PPAR $\gamma$ in the modulation of CRH using isolated mouse hearts. Our data demonstrated that: 1) Targeting sEH, through pharmacologic inhibition or genetic deletion, enhanced $\mathrm{CRH}$; 2) Disruption of sEH significantly changed oxylipin profiles, including an increased EET/DHET ratio, increased EpOME/DiHOME ratio, increased HODEs, decreased mid-chain HETEs, and decreased prostanoids; 3) Inhibition of CYP epoxygenases pathway attenuated CRH; and 4) The PPAR $\gamma$ receptors are involved in $\mathrm{CRH}$ as evident by the effect of PPAR $\gamma$-antagonist and PPAR $\gamma$-gonist used.

Disruption of $\mathrm{sEH}$ by pharmacologic inhibition ( $t$-AUCB) or genetic deletion $\left(\mathrm{sEH}^{-/-}\right)$ enhanced $\mathrm{CRH}$ after brief ischemia compared to control mice. $\mathrm{CRH}$ may be viewed as a protective mechanism, and is attenuated in pathologic conditions. The association between compromised CRH and some cardiovascular pathologies, such as cardiac hypertrophy (36), metabolic syndrome (6), unstable angina, myocardial infarction, congestive heart failure and cardiac death (30) confirms the significance of CRH. Further, the disrupting CRH was accompanied by significant increase in the measured EET and reduction in the corresponding 
DHETs. The rapid hydration of EETs (24) may explain the challenge of detecting them. EETs are formed by the epoxidation of arachidonic acid (AA) by CYP epoxygenases and possess well-established beneficial cardiovascular effects $(19,53,59,85,102)$. Deletion or inhibition of sEH, the main catabolic enzyme of EETs, has been widely used to increase the level of EETs and to investigate their effects (45). Acute inhibition of sEH, through injection of a sEH inhibitor, lowered blood pressure in spontaneously hypertensive rats (SHR) (101), whereas chronic inhibition of sEH lowered angiotensin-II-induced hypertension (34). Besides lowering blood pressure, sEH inhibition ameliorated renal damage in angiotensin-dependent, salt-sensitive hypertension rats (35). Also, deletion of sEH gene increases endogenous EETs by decreasing the metabolic conversion of EETs into DHETs. The observed CRH increase in mice due to sEH disruption through pharmacology or gene deletion was associated with an increase in 14,15-EET/14,15-DHET ratio without changing basic cardiac functions.

The brief ischemia had no effect on EET or DHET levels, which could be due to the short duration of ischemia (15 seconds). In contrast, DHETs (EETs metabolites) were elevated in the mouse heart perfusate in response to longer (20-min) ischemia (17).

Mid-chain (5-, 11-, 12- and 15-) HETE levels decreased in response to ischemia and to sEH-inhibition $t$-AUCB treatment, but not in $\mathrm{sEH}^{--}$. Mid-chain HETEs are produced through allylic oxidation of AA by lipoxygenase (LOX) (39). Increased formation of these mid-chain HETEs is involved in cardiovascular dysfunction through endothelial cells, smooth muscle cells, and monocytes $(13,68,87,97)$. They were shown to have chemotaxis effects, change vascular tone, and induce the production of vascular endothelial growth factors $(29,56,86,93)$. Unlike the vasodilatory effect of EETs in the kidneys (33), 12-HETE caused vasoconstriction in small renal arteries (48). Also, the generation of mid-chain HETEs is increased in essential hypertension (16) 
suggesting that they could be involved in its pathogenesis. These reports point to opposite effects of EETs and HETEs in vascular biology. Maayah et al. reported that mid-chain HETEs blocked the synthesis of EETs and increased their conversion to DHETs in RL-14 cells (49). Moreover, although sEH is not directly involved in the generation or breakdown of mid-chain HETEs, sEH was found to be essential for mid-chain HETE-mediated induction of cellular hypertrophy (49). Therefore, not only do EETs and mid-chain HETEs have opposite effects, they seem to affect the level of each other. In contrast to our findings, Li et al. reported that the plasma levels of 5-, 11-, 12-, and 15-HETE were not changed by sEH-inhibition in WT mice (45). However, their results were based on plasma samples, whereas ours were based on isolated heart perfusate samples, and demonstrated significant decrease in the level of 5-, 11-, 12-, and 15-HETEs in response to sEH inhibition. Also, Li et al. reported the plasma levels of 5-, 9-, 11-, and 15-HETEs were elevated in $\mathrm{sEH}^{-/-}$compared to $\mathrm{SEH}^{+/+}$mice (45). Again, this discrepancy could be explained by the different source of the samples; ours were from the isolated heart perfusate, whereas theirs were from the plasma (45). Based on our findings, the decrease in mid-chain HETEs in response to ischemia suggests that these metabolites are down regulated in response to ischemic episodes. This is an interesting finding because, out of all oxylipins measured, HETEs were the only oxylipins whose levels were affected by the brief ischemia.

Linoleic acid (LA) epoxidation metabolites, such as 9,10-, and 12,13-EpOMEs, and their corresponding sEH-metabolized DiHOMEs were detected in the heart perfusate samples. As expected, targeting sEH through inhibition (by $t$-AUCB) or deletion $\left(\mathrm{sEH}^{--}\right)$increased EpOME/DiHOME ratio. This resulted from increased 9,10-,12,13-EpOMEs, and decreased 9,10, 12,13-DiHOMEs. The physiological significance of EpOMEs and DiHOMEs remains poorly understood, and the evidence is somewhat contradictory, with a few studies suggesting toxic 
effects, while others indicating beneficial effects of these bioactive metabolites. EpOMEs and DiHOMEs were reported to increase oxidative stress in vascular endothelial cells (96); DiHOMEs were toxic to renal proximal tubular cells (54); and intravenously injected 9,10EpOME had cardiodepressive effects in dogs (90). The main caveat of these studies, which reported that EpOMEs and DiHOMEs had toxic effects, is that very high concentrations (100 $500 \mu \mathrm{M}$ ) were used (39). Contrary to these reports, lower, more physiological concentrations of EpOMEs and DiHOMEs did not have toxic effects and rather had beneficial effects (52). Mitchell et al reported that LA and its oxidative metabolites (EpOMEs and DiHOMEs) did not have toxic effects during acute exposure in Langendorff-perfused rat hearts (52). Pretreatment with 12,13-EpOME protected primary cultures of rabbit renal proximal tubular cells against hypoxia/reoxygenation injury (65). Also, increased EpOME/DiHOME ratio, induced by sEH inhibition using AUDA, improved renal recovery in response to ischemia/reperfusion injury in C57BL/6 mice (44). Based on these findings and on the described biologic role of LA epoxides, the increase in EpOME/DiHOME ratio may have contributed to enhancing $\mathrm{CRH}$.

Linoleic acid (LA) is also metabolized through hydroxylation by CYP epoxygenases to form hydroxyl-LA metabolites known as hydroxyoctadecadienoic acids (HODEs) (39). The detected 13-HODE was increased in $t$-AUCB-treated and $\mathrm{sEH}^{--}$mice versus corresponding controls. However, 9-HODE increased in $t$-AUCB-treated mice, but didn't change in $\mathrm{sEH}^{-1-}$ vs. $\mathrm{sEH}^{+/+}$mice. 9-HODE is considered pro-inflammatory, activating $\mathrm{G}$ protein coupled receptor and JNK $(26,66)$. Similar to EpOMEs, the physiologic function of HODEs is still being investigated (39). 13-HODE is suggested to have an anti-inflammatory role in inflammatory diseases through its effect as a PPAR $\gamma$-agonist $(2,4,18,21,88)$. Also, 13-HODE, through increasing prostacyclin 
$\left(\mathrm{PGI}_{2}\right)$ biosynthesis, was involved in splenic and coronary artery relaxation in smooth muscle cells in Mongrel dogs (15). 9-HODE, unlike 13-HODE, was described as pro-inflammatory in an experimental wound-healing model in rats $(26,66)$. In contrast to our data, Luria et al. reported no change in 9- and 13-HODE in $\mathrm{sEH}^{-/-}$compared to $\mathrm{sEH}^{+/+}$(47). This discrepancy may be explained by the difference in the source of the metabolites: we used heart perfusate samples, whereas Luria et al (47) used urinary samples. Also, the reported metabolic and functional effects of $\mathrm{sEH}^{-/-}$(genetic deletion of $\mathrm{sEH}$ ) and pharmacologic inhibition of $\mathrm{sEH}$ are not necessarily the same (45). Therefore, based on our results, the increase in 13-HODE in sEHdisrupted heart perfusates might have played a role in enhancing $\mathrm{CRH}$, whereas the role of 9HODE is not yet known based on its described biologic functions.

sEH inhibition with $t$-AUCB decreased prostanoid levels, including PGs and $\mathrm{TxB}_{2}$. PG-G and PG- $\mathrm{H}_{2}$, AA metabolites formed by $\mathrm{COX}$ isoforms (1 and 2), are converted to the 4 main bioactive PGs (PG-D 2 , PG-E 2 , PG- $\mathrm{I}_{2}$, PG-F $2 \alpha$ and thromboxanes $\left(\mathrm{TxA}_{2}\right.$ and $\left.\mathrm{TxB}_{2}\right)(74,92)$. Most PGs have pro-inflammatory effects. For example, $\mathrm{PG}-\mathrm{E}_{2}$ augments arterial dilation and increases microvascular permeability (92). However, PG-E $\mathrm{E}_{2}$ was found to have an anti-inflammatory role as well by up-regulating cAMP and inducing secretion of the anti-inflammatory IL-10 (78). Similarly, PG-D 2 attenuated inflammation in experimental models of pleuritis and colitis (92). 6keto-PG-F $\mathrm{F}_{1 \alpha}$, the non-active hydrolysis product of prostacyclin $\left(\mathrm{PG}-\mathrm{I}_{2}\right)$, and $\mathrm{TxB}_{2}$, the inactive degradation product of $\mathrm{TxA}_{2}(92)$, were also decreased by $t$-AUCB. PG-F $\mathrm{F}_{2 \alpha}$ is elevated in patients with chronic inflammatory diseases (92). In support of our findings, sEH-inhibition by AUDABE reversed lipopolysaccharide (LPS)-induced increase in PG- $\mathrm{D}_{2}, \mathrm{PG}-\mathrm{E}_{2}, 6-$ keto-PG-F $\mathrm{F}_{1 \alpha}$, and $\mathrm{TxB}_{2}$ (76). Also, sEH-inhibition by AUDA-BE, through increasing the concentrations of EETs, 
inhibited NF- $\mathrm{KB}$ translocation (75). Combination of sEH-inhibition by AUDA-BE and COXinhibition had an additive effect and further decreased PG- $\mathrm{D}_{2}$ and PG- $\mathrm{E}_{2}(75)$. Therefore, the associated decrease in the measured PGs could be attributed to sEH-inhibition by $t$-AUCB. However, the impact of these changes on the $t$-AUCB-enhanced CRH is not yet clear.

As mentioned earlier, EETs are generated from AA by cytochrome P450 (CYP) epoxygenase enzymes, primarily CYP2C and CYP2J. The CYP epoxygenase-EET pathway was targeted using pharmacologic inhibition of CYP-epoxygenases to further support our findings of the involvement of CYP-epoxygenases in modulating CRH. sEH-inhibition increases the level of EETs by blocking their breakdown; we investigated the effect of blocking the synthesis of EETs on CRH using a CYP epoxygenases-inhibitor. Evidently, inhibiting CYP epoxygenases would negatively impact EET synthesis and, therefore, their effects. CYP450 enzymes are highest in coronary arteries and in small arterioles (20). MS-PPOH was used as an EET-synthesis inhibitor that blocked the role of EETs as mediators of insulin-mediated augmentation of skeletal muscle perfusion (82). Our data indicate that MS-PPOH significantly decreased CRH after a brief ischemia. These findings suggest that inhibition of CYP epoxygenases by MS-PPOH decreased CRH possibly due to inhibition of EETs synthesis. Further studies to investigate the impact of MS-PPOH on oxylipin profiles are needed to understand the metabolic changes associated with the inhibition of CYP epoxygenases.

Also, we investigated the relationship between CRH and PPAR $\gamma$. Our data showed that $\mathrm{CRH}$ was attenuated in $\mathrm{sEH}^{-/}$, WT $\left(\mathrm{sEH}^{++}\right)$, and $t$-AUCB-treated WT mouse hearts by T0070907 (PPAR $\gamma$-antagonist), but enhanced by rosiglitazone (PPAR $\gamma$-agonist). These results from PPAR $\gamma$-antagonist and $\operatorname{PPAR} \gamma$-agonist experiments suggest that $\operatorname{PPAR} \gamma$ receptors are 
involved in modulating CRH. Interestingly, studies indicated that EET effects could be mediated by PPAR $\gamma$ receptors $(8,14,46,70)$. Our lab reported that $\mathrm{sEH}^{-/-}$mouse aorta had increased expression of PPAR $\gamma$ protein and enhanced vascular relaxation compared to $\mathrm{sEH}^{+/+}$ mice (59). Liu et al. suggested that selective sEH inhibition potentiated the anti-inflammatory effect in endothelial cells, presumably by increasing the retention of EETs, which would activate PPAR $\gamma$ (46). These studies indicate that EETs' effects could be mediated by PPAR $\gamma$ receptors. Also, the attenuated $t$-AUCB-enhanced CRH in WT mice (by T0070907) suggests that PPAR $\gamma$ may mediate CRH downstream of CYP epoxygenases-EET pathway.

Infusion of L-NAME, a non-selective NO synthase inhibitor, decreased CRH in both $\mathrm{sEH}^{+/+}$and $\mathrm{sEH}^{-/-}$mice. These data suggest that the presence or absence of $\mathrm{sEH}$ does not have any specific effect on L-NAME-induced attenuation of CRH response in both $\mathrm{sEH}^{+/+}$and $\mathrm{sEH}^{-/-}$ mice. Similarly, Zhang et al. described that the inhibition of the activity of sEH enzyme ameliorates endothelial dysfunction in the $d b / d b$ mice, which is NO-independent, but dependent on cyp450-epoxygenase-derived metabolites (EETs) (103). Our studies have shown that vascular relaxation through $\mathrm{A}_{2 \mathrm{~A}} \mathrm{AR}$ was independent of $\mathrm{NO}$ and cyclooxygenase pathways (57, 58, $60)$. Also, others $(17,39,45)$ reported that $\mathrm{sEH}^{-/-}$mice were associated with elevated EETs levels compared to $\mathrm{sEH}^{+/+}$mice, and these EETs exerted cardioprotective effects against ischemia/reperfusion injury (77). Therefore, the enhanced $\mathrm{CRH}$ in $\mathrm{sEH}^{-/-}$compared to $\mathrm{sEH}^{+/+}$ mice seems to be independent of the NO pathway, but dependent on the changes associated with sEH-deletion. Although cyclooxygenase (COX) pathway was found to be involved in myocardial reactive hyperemia in dogs, and a cross-talk between COX and nitric oxide pathways in dog coronary vessels was suggested (72), a later publication by Hellmann et al. (27) suggested that COX-metabolites, prostanoids, were not involved in reactive hyperemia in human skin. 
Accordingly, the role of prostanoids in mouse $\mathrm{CRH}$ was not investigated in our study, and therefore, cannot be ruled out.

\section{Summary and Conclusions:}

Ischemia continues to pose significant health risks to people in both developed and developing countries. The heart is mainly affected in ischemic heart disease (IHD), which has been a leading cause of morbidity and mortality of long-standing industrialized countries, and in countries with emerging economies (7). Consequences of ischemia on the heart range from functional and structural damage to infarction and death. Physiologically, reperfusion after a period of ischemia is accompanied by increased nourishment in what seems to be an attempt to speed up recovery and to decrease the potential ischemia-induced damage. This increased nourishment is known as coronary reactive hyperemia $(\mathrm{CRH})$. Extensive research has been dedicated to understanding the mechanisms and mediators of CRH. The association between compromised $\mathrm{CRH}$ and some cardiovascular pathologies $(6,30,36)$ confirms the significance of CRH.

The confirmed beneficial effects of EETs, as shown in animal and human epidemiological studies, such as cardioprotection against in ischemia / reperfusion injury (77), and vasodilation $(23,33,71)$ were the basis for our project. sEH is a key element in the biologic roles of EETs. It has been targeted, through deletion or inhibition, to study the effects of EETs. However, studies have also revealed that when sEH was targeted, other oxylipins were also affected. Oxylipins have numerous biologic effects which are still to be uncovered.

Our findings demonstrate that targeting sEH, through pharmacologic inhibition or genetic deletion, is associated with enhanced CRH, and with significant changes in arachidonic acid- 
derived metabolites (EETs, prostanoids, and HETEs), and linoleic acid-derived metabolites (EpOMEs, DiHOMEs, and HODEs). The enhanced CRH associated with disruption of sEH could be through increased EET/DHET ratio, increased 13-HODE, and increased EpOMEs/DiHOMEs ratio. Also, PPAR $\gamma$ activation mediates $\mathrm{CRH}$ downstream of the CYP epoxygenases-EET pathway. Therefore, targeting sEH through pharmacology could be a feasible option for clinical implementation in cardiovascular diseases in which the hyperemic response is blunted or compromised. 


\section{FUTURE DIRECTIONS}

This research opens new prospects for combining functional and biochemical data in trying to better understand the impact of ischemia on cardiac function and the effect of some lipid mediators on modulating that response. It certainly contributes to our understanding of the complex changes in many of the oxylipins in response to pharmacologic intervention or genetic changes; however, it does create more questions regarding the exact role each of the measured oxylipins play in the cardiac response to ischemic insults. More studies need to be done to assess the effect of these different oxylpins on CRH through the use of agonists or antagonists. Luckily, genetic models, which provide specific focus on enzymes involved in the formation or breakdown of EETs. Such models include endothelial over-expression of CYP2J2 (Tie2-CYP2J2 Tr.) and endothelial over-expression sEH (Tie2-sEH Tr.). Future studies to investigate coronary reactive hyperemia in these models compared to wild type need to be performed. Additionally, oxylipins profiling in these models (Tie2-CYP2J2 Tr. and Tie2-sEH Tr.) will also improve our understanding of how oxylipins change and what role they might play when different enzymes are affected under ischemic conditions. 


\section{REFERENCES}

1. Al-Shabrawey M, Mussell R, Kahook K, Tawfik A, Eladl M, Sarthy V, Nussbaum J, El-Marakby A, Park SY, Gurel Z, Sheibani N, and Maddipati KR. Increased expression and activity of 12-lipoxygenase in oxygen-induced ischemic retinopathy and proliferative diabetic retinopathy: implications in retinal neovascularization. Diabetes 60: 614-624, 2011.

2. Altmann R, Hausmann M, Spottl T, Gruber M, Bull AW, Menzel K, Vogl D, Herfarth H, Scholmerich J, Falk W, and Rogler G. 13-Oxo-ODE is an endogenous ligand for PPARgamma in human colonic epithelial cells. Biochem Pharmacol 74: 612-622, 2007.

3. Askari AA, Thomson S, Edin ML, Lih FB, Zeldin DC, and Bishop-Bailey D. Basal and inducible anti-inflammatory epoxygenase activity in endothelial cells. Biochem Biophys Res Commun 446: 633-637, 2014.

4. Belvisi MG and Mitchell JA. Targeting PPAR receptors in the airway for the treatment of inflammatory lung disease. Br J Pharmacol 158: 994-1003, 2009.

5. Berwick ZC, Payne GA, Lynch B, Dick GM, Sturek M, and Tune JD. Contribution of adenosine $\mathrm{A}(2 \mathrm{~A})$ and $\mathrm{A}(2 \mathrm{~B})$ receptors to ischemic coronary dilation: role of $\mathrm{K}(\mathrm{V})$ and K(ATP) channels. Microcirculation 17: 600-607, 2010.

6. Borbouse L, Dick GM, Payne GA, Berwick ZC, Neeb ZP, Alloosh M, Bratz IN, Sturek M, and Tune JD. Metabolic syndrome reduces the contribution of $\mathrm{K}+$ channels to ischemic coronary vasodilation. Am J Physiol Heart Circ Physiol 298: 29, 2010.

7. Buja LM and Vander Heide RS. Pathobiology of Ischemic Heart Disease: Past, Present and Future. Cardiovasc Pathol 25: 214-220, 2016. 
8. Cai Z, Zhao G, Yan J, Liu W, Feng W, Ma B, Yang L, Wang JA, Tu L, and Wang DW. CYP2J2 overexpression increases EETs and protects against angiotensin II-induced abdominal aortic aneurysm in mice. J Lipid Res 54: 1448-1456, 2013.

9. Campbell WB, Gebremedhin D, Pratt PF, and Harder DR. Identification of epoxyeicosatrienoic acids as endothelium-derived hyperpolarizing factors. Circ Res 78: 415-423, 1996.

10. Catella F, Lawson JA, Fitzgerald DJ, and FitzGerald GA. Endogenous biosynthesis of arachidonic acid epoxides in humans: increased formation in pregnancy-induced hypertension. Proc Natl Acad Sci U S A 87: 5893-5897, 1990.

11. Chinetti G, Fruchart JC, and Staels B. Peroxisome proliferator-activated receptors (PPARs): nuclear receptors with functions in the vascular wall. Z Kardiol 3: 125-132, 2001.

12. Coffman JD and Gregg DE. Reactive hyperemia characteristics of the myocardium. Am J Physiol 199: 1143-1149, 1960.

13. Conrad DJ, Kuhn H, Mulkins M, Highland E, and Sigal E. Specific inflammatory cytokines regulate the expression of human monocyte 15-lipoxygenase. Proc Natl Acad Sci U S A 89: 217-221, 1992.

14. Cowart LA, Wei S, Hsu MH, Johnson EF, Krishna MU, Falck JR, and Capdevila JH. The CYP4A isoforms hydroxylate epoxyeicosatrienoic acids to form high affinity peroxisome proliferator-activated receptor ligands. J Biol Chem 277: 35105-35112, 2002.

15. De Meyer GR, Bult H, Verbeuren TJ, and Herman AG. The role of endothelial cells in the relaxations induced by 13-hydroxy- and 13-hydroperoxylinoleic acid in canine arteries. $\mathrm{Br}$ J Pharmacol 107: 597-603, 1992. 
16. Dolegowska B, Blogowski W, Kedzierska K, Safranow K, Jakubowska K, Olszewska M, Rac M, Chlubek D, and Ciechanowski K. Platelets arachidonic acid metabolism in patients with essential hypertension. Platelets 20: 242-249, 2009.

17. Edin ML, Wang Z, Bradbury JA, Graves JP, Lih FB, DeGraff LM, Foley JF, Torphy R, Ronnekleiv OK, Tomer KB, Lee CR, and Zeldin DC. Endothelial expression of human cytochrome P450 epoxygenase CYP2C8 increases susceptibility to ischemia-reperfusion injury in isolated mouse heart. Faseb J 25: 3436-3447, 2011.

18. Emerson MR and LeVine SM. Experimental allergic encephalomyelitis is exacerbated in mice deficient for 12/15-lipoxygenase or 5-lipoxygenase. Brain Res 17: 140-145, 2004.

19. Fang X, Kaduce TL, Weintraub NL, Harmon S, Teesch LM, Morisseau C, Thompson DA, Hammock BD, and Spector AA. Pathways of epoxyeicosatrienoic acid metabolism in endothelial cells. Implications for the vascular effects of soluble epoxide hydrolase inhibition. J Biol Chem 276: 14867-14874, 2001.

20. Fleming I. The pharmacology of the cytochrome P450 epoxygenase/soluble epoxide hydrolase axis in the vasculature and cardiovascular disease. Pharmacol Rev 66: 1106-1140, 2014.

21. Fritsche KL. Too much linoleic acid promotes inflammation-doesn't it? Prostaglandins Leukot Essent Fatty Acids 79: 173-175, 2008.

22. Gabbs M, Leng S, Devassy JG, Monirujjaman M, and Aukema HM. Advances in Our Understanding of Oxylipins Derived from Dietary PUFAs. Adv Nutr 6: 513-540, 2015.

23. Gebremedhin D, Ma YH, Falck JR, Roman RJ, VanRollins M, and Harder DR. Mechanism of action of cerebral epoxyeicosatrienoic acids on cerebral arterial smooth muscle. Am J Physiol 263: H519-525, 1992. 
24. Gilroy DW, Edin ML, De Maeyer RP, Bystrom J, Newson J, Lih FB, Stables M, Zeldin DC, and Bishop-Bailey D. CYP450-derived oxylipins mediate inflammatory resolution. Proc Natl Acad Sci U S A 25: 201521453, 2016.

25. Gleim S, Stitham J, Tang WH, Martin KA, and Hwa J. An eicosanoid-centric view of atherothrombotic risk factors. Cell Mol Life Sci 69: 3361-3380, 2012.

26. Hattori T, Obinata H, Ogawa A, Kishi M, Tatei K, Ishikawa O, and Izumi T. G2A plays proinflammatory roles in human keratinocytes under oxidative stress as a receptor for 9hydroxyoctadecadienoic acid. J Invest Dermatol 128: 1123-1133, 2008.

27. Hellmann M, Gaillard-Bigot F, Roustit M, and Cracowski JL. Prostanoids are not involved in postocclusive reactive hyperaemia in human skin. Fundam Clin Pharmacol 29: 510516, 2015.

28. Hercule HC, Schunck WH, Gross V, Seringer J, Leung FP, Weldon SM, da Costa Goncalves A, Huang Y, Luft FC, and Gollasch M. Interaction between P450 eicosanoids and nitric oxide in the control of arterial tone in mice. Arterioscler Thromb Vasc Biol 29: 54-60, 2009.

29. Honda HM, Leitinger N, Frankel M, Goldhaber JI, Natarajan R, Nadler JL, Weiss JN, and Berliner JA. Induction of monocyte binding to endothelial cells by MM-LDL: role of lipoxygenase metabolites. Arterioscler Thromb Vasc Biol 19: 680-686, 1999.

30. Huang AL, Silver AE, Shvenke E, Schopfer DW, Jahangir E, Titas MA, Shpilman A, Menzoian JO, Watkins MT, Raffetto JD, Gibbons G, Woodson J, Shaw PM, Dhadly M, Eberhardt RT, Keaney JF, Jr., Gokce N, and Vita JA. Predictive value of reactive hyperemia for cardiovascular events in patients with peripheral arterial disease undergoing vascular surgery. Arterioscler Thromb Vasc Biol 27: 2113-2119, 2007. 
31. Hwang J, Kleinhenz DJ, Lassegue B, Griendling KK, Dikalov S, and Hart CM. Peroxisome proliferator-activated receptor-gamma ligands regulate endothelial membrane superoxide production. Am J Physiol Cell Physiol 288: 8, 2005.

32. Imig JD. Epoxides and soluble epoxide hydrolase in cardiovascular physiology. Physiol Rev 92: 101-130, 2012.

33. Imig JD, Navar LG, Roman RJ, Reddy KK, and Falck JR. Actions of epoxygenase metabolites on the preglomerular vasculature. J Am Soc Nephrol 7: 2364-2370, 1996.

34. Imig JD, Zhao X, Capdevila JH, Morisseau C, and Hammock BD. Soluble epoxide hydrolase inhibition lowers arterial blood pressure in angiotensin II hypertension. Hypertension 39: 690-694, 2002.

35. Imig JD, Zhao X, Zaharis CZ, Olearczyk JJ, Pollock DM, Newman JW, Kim IH, Watanabe T, and Hammock BD. An orally active epoxide hydrolase inhibitor lowers blood pressure and provides renal protection in salt-sensitive hypertension. Hypertension 46: 975-981, 2005.

36. Kingsbury MP, Turner MA, Flores NA, Bovill E, and Sheridan DJ. Endogenous and exogenous coronary vasodilatation are attenuated in cardiac hypertrophy: a morphological defect? J Mol Cell Cardiol 32: 527-538, 2000.

37. Kitakaze M, Hori M, Takashima S, Iwai K, Sato H, Inoue M, Kitabatake A, and Kamada T. Superoxide dismutase enhances ischemia-induced reactive hyperemic flow and adenosine release in dogs. A role of 5'-nucleotidase activity. Circ Res 71: 558-566, 1992.

38. Klein LW, Agarwal JB, Schneider RM, Hermann G, Weintraub WS, and Helfant RH. Effects of previous myocardial infarction on measurements of reactive hyperemia and the coronary vascular reserve. J Am Coll Cardiol 8: 357-363, 1986. 
39. Konkel A and Schunck WH. Role of cytochrome P450 enzymes in the bioactivation of polyunsaturated fatty acids. Biochim Biophys Acta 1: 210-222, 2011.

40. Lagarde M, Bernoud-Hubac N, Calzada C, Vericel E, and Guichardant $M$. Lipidomics of essential fatty acids and oxygenated metabolites. Mol Nutr Food Res 57: 13471358, 2013.

41. Lee CR, Imig JD, Edin ML, Foley J, DeGraff LM, Bradbury JA, Graves JP, Lih FB, Clark J, Myers P, Perrow AL, Lepp AN, Kannon MA, Ronnekleiv OK, Alkayed NJ, Falck JR, Tomer KB, and Zeldin DC. Endothelial expression of human cytochrome P450 epoxygenases lowers blood pressure and attenuates hypertension-induced renal injury in mice. Faseb J 24: 3770-3781, 2010.

42. Lee CR, North KE, Bray MS, Couper DJ, Heiss G, and Zeldin DC. CYP2J2 and CYP2C8 polymorphisms and coronary heart disease risk: the Atherosclerosis Risk in Communities (ARIC) study. Pharmacogenet Genomics 17: 349-358, 2007.

43. Lee CR, North KE, Bray MS, Fornage M, Seubert JM, Newman JW, Hammock BD, Couper DJ, Heiss G, and Zeldin DC. Genetic variation in soluble epoxide hydrolase (EPHX2) and risk of coronary heart disease: The Atherosclerosis Risk in Communities (ARIC) study. Hum Mol Genet 15: 1640-1649, 2006.

44. Lee JP, Yang SH, Lee HY, Kim B, Cho JY, Paik JH, Oh YJ, Kim DK, Lim CS, and Kim YS. Soluble epoxide hydrolase activity determines the severity of ischemia-reperfusion injury in kidney. PLoS One 7: 10, 2012.

45. Li L, Li N, Pang W, Zhang X, Hammock BD, Ai D, and Zhu Y. Opposite effects of gene deficiency and pharmacological inhibition of soluble epoxide hydrolase on cardiac fibrosis. PLoS One 9, 2014. 
46. Liu Y, Zhang Y, Schmelzer K, Lee TS, Fang X, Zhu Y, Spector AA, Gill S, Morisseau C, Hammock BD, and Shyy JY. The antiinflammatory effect of laminar flow: the role of PPARgamma, epoxyeicosatrienoic acids, and soluble epoxide hydrolase. Proc Natl Acad Sci U S A 102: 16747-16752, 2005.

47. Luria A, Weldon SM, Kabcenell AK, Ingraham RH, Matera D, Jiang H, Gill R, Morisseau C, Newman JW, and Hammock BD. Compensatory mechanism for homeostatic blood pressure regulation in Ephx2 gene-disrupted mice. J Biol Chem 282: 2891-2898, 2007.

48. Ma YH, Harder DR, Clark JE, and Roman RJ. Effects of 12-HETE on isolated dog renal arcuate arteries. Am J Physiol 261: H451-456, 1991.

49. Maayah ZH and El-Kadi AO. 5-, 12- and 15-Hydroxyeicosatetraenoic acids induce cellular hypertrophy in the human ventricular cardiomyocyte, RL-14 cell line, through MAPKand NF-kappaB-dependent mechanism. Arch Toxicol 90: 359-373, 2016.

50. Massey KA and Nicolaou A. Lipidomics of oxidized polyunsaturated fatty acids. Free Radic Biol Med 59: 45-55, 2013.

51. Michalik L, Auwerx J, Berger JP, Chatterjee VK, Glass CK, Gonzalez FJ, Grimaldi PA, Kadowaki T, Lazar MA, O'Rahilly S, Palmer CN, Plutzky J, Reddy JK, Spiegelman BM, Staels B, and Wahli W. International Union of Pharmacology. LXI. Peroxisome proliferator-activated receptors. Pharmacol Rev 58: 726-741, 2006.

52. Mitchell LA, Grant DF, Melchert RB, Petty NM, and Kennedy RH. Linoleic acid metabolites act to increase contractility in isolated rat heart. Cardiovasc Toxicol 2: 219-230, 2002. 
53. Moghaddam MF, Grant DF, Cheek JM, Greene JF, Williamson KC, and Hammock

BD. Bioactivation of leukotoxins to their toxic diols by epoxide hydrolase. Nat Med 3: 562-566, 1997.

54. Moran JH, Weise R, Schnellmann RG, Freeman JP, and Grant DF. Cytotoxicity of linoleic acid diols to renal proximal tubular cells. Toxicol Appl Pharmacol 146: 53-59, 1997.

55. Morisseau C and Hammock BD. Epoxide hydrolases: mechanisms, inhibitor designs, and biological roles. Annu Rev Pharmacol Toxicol 45: 311-333, 2005.

56. Nakao J, Ooyama T, Ito H, Chang WC, and Murota S. Comparative effect of lipoxygenase products of arachidonic acid on rat aortic smooth muscle cell migration. Atherosclerosis 44: 339-342, 1982.

57. Nayeem MA, Poloyac SM, Falck JR, Zeldin DC, Ledent C, Ponnoth DS, Ansari HR, and Mustafa SJ. Role of CYP epoxygenases in A2A AR-mediated relaxation using A2A ARnull and wild-type mice. Am J Physiol Heart Circ Physiol 295: 19, 2008.

58. Nayeem MA, Ponnoth DS, Boegehold MA, Zeldin DC, Falck JR, and Mustafa SJ. High-salt diet enhances mouse aortic relaxation through adenosine A2A receptor via CYP epoxygenases. Am J Physiol Regul Integr Comp Physiol 296: 24, 2009.

59. Nayeem MA, Pradhan I, Mustafa SJ, Morisseau C, Falck JR, and Zeldin DC. Adenosine A2A receptor modulates vascular response in soluble epoxide hydrolase-null mice through CYP-epoxygenases and PPARgamma. Am J Physiol Regul Integr Comp Physiol 304: 14, 2013.

60. Nayeem MA, Zeldin DC, Boegehold MA, and Falck JR. Salt modulates vascular response through adenosine $\mathrm{A}(2 \mathrm{~A})$ receptor in eNOS-null mice: role of CYP450 epoxygenase and soluble epoxide hydrolase. Mol Cell Biochem 350: 101-111, 2011. 
61. Nayeem MA, Zeldin DC, Boegehold MA, Morisseau C, Marowsky A, Ponnoth DS, Roush KP, and Falck JR. Modulation by salt intake of the vascular response mediated through adenosine $\mathrm{A}(2 \mathrm{~A})$ receptor: role of CYP epoxygenase and soluble epoxide hydrolase. Am $J$ Physiol Regul Integr Comp Physiol 299: 28, 2010.

62. Newman JW, Morisseau C, and Hammock BD. Epoxide hydrolases: their roles and interactions with lipid metabolism. Prog Lipid Res 44: 1-51, 2005.

63. Node K, Ruan XL, Dai J, Yang SX, Graham L, Zeldin DC, and Liao JK. Activation of Galpha s mediates induction of tissue-type plasminogen activator gene transcription by epoxyeicosatrienoic acids. J Biol Chem 276: 15983-15989, 2001.

64. Nolte RT, Wisely GB, Westin S, Cobb JE, Lambert MH, Kurokawa R, Rosenfeld MG, Willson TM, Glass CK, and Milburn MV. Ligand binding and co-activator assembly of the peroxisome proliferator-activated receptor-gamma. Nature 395: 137-143, 1998.

65. Nowak G, Grant DF, and Moran JH. Linoleic acid epoxide promotes the maintenance of mitochondrial function and active $\mathrm{Na}+$ transport following hypoxia. Toxicol Lett 147: 161$175,2004$.

66. Obinata $\mathbf{H}$ and Izumi T. G2A as a receptor for oxidized free fatty acids. Prostaglandins Other Lipid Mediat 89: 66-72, 2009.

67. Park S, Lim S, Chang W, Song H, Lee S, Song BW, Kim HJ, Cha MJ, Choi E, Jang Y, Chung N, Cho SY, and Hwang KC. The inhibition of insulin-stimulated proliferation of vascular smooth muscle cells by rosiglitazone is mediated by the Akt-mTOR-P70S6K pathway. Yonsei Med J 49: 592-600, 2008. 
68. Patricia MK, Kim JA, Harper CM, Shih PT, Berliner JA, Natarajan R, Nadler JL, and Hedrick CC. Lipoxygenase products increase monocyte adhesion to human aortic endothelial cells. Arterioscler Thromb Vasc Biol 19: 2615-2622, 1999.

69. Ponnoth DS, Nayeem MA, Tilley SL, Ledent C, and Jamal Mustafa S. CYPepoxygenases contribute to A2A receptor-mediated aortic relaxation via sarcolemmal KATP channels. Am J Physiol Regul Integr Comp Physiol 303: 26, 2012.

70. Pradhan I, Ledent C, Mustafa SJ, Morisseau C, and Nayeem MA. High salt diet modulates vascular response in $\mathrm{AAR}$ and $\mathrm{A}$ AR mice: role of sEH, PPARgamma, and $\mathrm{K}$ channels. Mol Cell Biochem 5: 5, 2015.

71. Proctor KG, Falck JR, and Capdevila J. Intestinal vasodilation by epoxyeicosatrienoic acids: arachidonic acid metabolites produced by a cytochrome P450 monooxygenase. Circ Res 60: 50-59, 1987.

72. Puybasset L, Bea ML, Ghaleh B, Giudicelli JF, and Berdeaux A. Coronary and systemic hemodynamic effects of sustained inhibition of nitric oxide synthesis in conscious dogs. Evidence for cross talk between nitric oxide and cyclooxygenase in coronary vessels. Circ Res 79: 343-357, 1996.

73. Qin J, Kandhi S, Froogh G, Jiang H, Luo M, Sun D, and Huang A. Sexually dimorphic phenotype of arteriolar responsiveness to shear stress in soluble epoxide hydrolaseknockout mice. Am J Physiol Heart Circ Physiol 309: 9, 2015.

74. Ricciotti E and FitzGerald GA. Prostaglandins and inflammation. Arterioscler Thromb Vasc Biol 31: 986-1000, 2011.

75. Schmelzer KR, Inceoglu B, Kubala L, Kim IH, Jinks SL, Eiserich JP, and Hammock BD. Enhancement of antinociception by coadministration of nonsteroidal anti- 
inflammatory drugs and soluble epoxide hydrolase inhibitors. Proc Natl Acad Sci U S A 103: 13646-13651, 2006.

76. Schmelzer KR, Kubala L, Newman JW, Kim IH, Eiserich JP, and Hammock BD. Soluble epoxide hydrolase is a therapeutic target for acute inflammation. Proc Natl Acad Sci U S A 102: 9772-9777, 2005.

77. Seubert J, Yang B, Bradbury JA, Graves J, Degraff LM, Gabel S, Gooch R, Foley J, Newman J, Mao L, Rockman HA, Hammock BD, Murphy E, and Zeldin DC. Enhanced postischemic functional recovery in CYP2J2 transgenic hearts involves mitochondrial ATPsensitive K+ channels and p42/p44 MAPK pathway. Circ Res 95: 506-514, 2004.

78. Sha W, Brune B, and Weigert A. The multi-faceted roles of prostaglandin E2 in cancerinfiltrating mononuclear phagocyte biology. Immunobiology 217: 1225-1232, 2012.

79. Shahabi P, Siest G, Meyer UA, and Visvikis-Siest S. Human cytochrome P450 epoxygenases: variability in expression and role in inflammation-related disorders. Pharmacol Ther 144: 134-161, 2014.

80. Sharifi-Sanjani M, Zhou X, Asano S, Tilley S, Ledent C, Teng B, Dick GM, and Mustafa SJ. Interactions between $\mathrm{A}(2 \mathrm{~A})$ adenosine receptors, hydrogen peroxide, and KATP channels in coronary reactive hyperemia. Am J Physiol Heart Circ Physiol 304: 22, 2013.

81. Shearer GC and Newman JW. Impact of circulating esterified eicosanoids and other oxylipins on endothelial function. Curr Atheroscler Rep 11: 403-410, 2009.

82. Shim CY, Kim S, Chadderdon S, Wu M, Qi Y, Xie A, Alkayed NJ, Davidson BP, and Lindner JR. Epoxyeicosatrienoic acids mediate insulin-mediated augmentation in skeletal muscle perfusion and blood volume. Am J Physiol Endocrinol Metab 307: 21, 2014. 
83. Sinal CJ, Miyata M, Tohkin M, Nagata K, Bend JR, and Gonzalez FJ. Targeted disruption of soluble epoxide hydrolase reveals a role in blood pressure regulation. J Biol Chem 275: 40504-40510, 2000.

84. Skrzypiec-Spring M, Grotthus B, Szelag A, and Schulz R. Isolated heart perfusion according to Langendorff---still viable in the new millennium. J Pharmacol Toxicol Methods 55: 113-126, 2007.

85. Spector AA, Fang X, Snyder GD, and Weintraub NL. Epoxyeicosatrienoic acids (EETs): metabolism and biochemical function. Prog Lipid Res 43: 55-90, 2004.

86. Stern N, Natarajan R, Tuck ML, Laird E, and Nadler JL. Selective inhibition of angiotensin-II-mediated aldosterone secretion by 5-hydroxyeicosatetraenoic acid. Endocrinology 125: 3090-3095, 1989.

87. Stern N, Yanagawa N, Saito F, Hori M, Natarajan R, Nadler J, and Tuck M. Potential role of 12 hydroxyeicosatetraenoic acid in angiotensin II-induced calcium signal in rat glomerulosa cells. Endocrinology 133: 843-847, 1993.

88. Stoll LL, Morland MR, and Spector AA. 13-HODE increases intracellular calcium in vascular smooth muscle cells. Am J Physiol 266: C990-996, 1994.

89. Strassburg K, Huijbrechts AM, Kortekaas KA, Lindeman JH, Pedersen TL, Dane A, Berger R, Brenkman A, Hankemeier T, van Duynhoven J, Kalkhoven E, Newman JW, and Vreeken RJ. Quantitative profiling of oxylipins through comprehensive LC-MS/MS analysis: application in cardiac surgery. Anal Bioanal Chem 404: 1413-1426, 2012.

90. Sugiyama S, Hayakawa M, Nagai S, Ajioka M, and Ozawa T. Leukotoxin, 9, 10epoxy-12-octadecenoate, causes cardiac failure in dogs. Life Sci 40: 225-231, 1987. 
91. Suryapranata H, Zijlstra F, MacLeod DC, van den Brand M, de Feyter PJ, and Serruys PW. Predictive value of reactive hyperemic response on reperfusion on recovery of regional myocardial function after coronary angioplasty in acute myocardial infarction. Circulation 89: 1109-1117, 1994.

92. Tam VC. Lipidomic profiling of bioactive lipids by mass spectrometry during microbial infections. Semin Immunol 25: 240-248, 2013.

93. Tang DG, Renaud C, Stojakovic S, Diglio CA, Porter A, and Honn KV. 12(S)-HETE is a mitogenic factor for microvascular endothelial cells: its potential role in angiogenesis. Biochem Biophys Res Commun 211: 462-468, 1995.

94. Thuresson ED, Lakkides KM, and Smith WL. Different catalytically competent arrangements of arachidonic acid within the cyclooxygenase active site of prostaglandin endoperoxide H synthase-1 lead to the formation of different oxygenated products. J Biol Chem 275: 8501-8507, 2000.

95. Tourdot BE, Ahmed I, and Holinstat M. The emerging role of oxylipins in thrombosis and diabetes. Front Pharmacol 4: 7, 2014.

96. Viswanathan S, Hammock BD, Newman JW, Meerarani P, Toborek M, and Hennig B. Involvement of CYP 2C9 in mediating the proinflammatory effects of linoleic acid in vascular endothelial cells. J Am Coll Nutr 22: 502-510, 2003.

97. Wen Y, Nadler JL, Gonzales N, Scott S, Clauser E, and Natarajan R. Mechanisms of ANG II-induced mitogenic responses: role of 12-lipoxygenase and biphasic MAP kinase. Am J Physiol 271: C1212-1220, 1996.

98. Wray J and Bishop-Bailey D. Epoxygenases and peroxisome proliferator-activated receptors in mammalian vascular biology. Exp Physiol 93: 148-154, 2008. 
99. Xu HE, Lambert MH, Montana VG, Plunket KD, Moore LB, Collins JL, Oplinger JA, Kliewer SA, Gampe RT, Jr., McKee DD, Moore JT, and Willson TM. Structural determinants of ligand binding selectivity between the peroxisome proliferator-activated receptors. Proc Natl Acad Sci U S A 98: 13919-13924, 2001.

100. Yang W, Tuniki VR, Anjaiah S, Falck JR, Hillard CJ, and Campbell WB. Characterization of epoxyeicosatrienoic acid binding site in U937 membranes using a novel radiolabeled agonist, 20-125i-14,15-epoxyeicosa-8(Z)-enoic acid. J Pharmacol Exp Ther 324: 1019-1027, 2008.

101. Yu Z, Xu F, Huse LM, Morisseau C, Draper AJ, Newman JW, Parker C, Graham L, Engler MM, Hammock BD, Zeldin DC, and Kroetz DL. Soluble epoxide hydrolase regulates hydrolysis of vasoactive epoxyeicosatrienoic acids. Circ Res 87: 992-998, 2000.

102. Zeldin DC. Epoxygenase pathways of arachidonic acid metabolism. J Biol Chem 276: 36059-36062, 2001.

103. Zhang LN, Vincelette J, Chen D, Gless RD, Anandan SK, Rubanyi GM, Webb HK, MacIntyre DE, and Wang YX. Inhibition of soluble epoxide hydrolase attenuates endothelial dysfunction in animal models of diabetes, obesity and hypertension. Eur J Pharmacol 654: 6874, 2011.

104. Zhao X, Du JQ, Xu DY, and Zhao SP. Effects of soluble epoxide hydrolase inhibitor on the expression of fatty acid synthase in peripheral blood mononuclear cell in patients with acute coronary syndrome. Lipids Health Dis 12: 12-13, 2013.

105. Zhou X, Teng B, Tilley S, and Mustafa SJ. A1 adenosine receptor negatively modulates coronary reactive hyperemia via counteracting A2A-mediated $\mathrm{H} 2 \mathrm{O} 2$ production and KATP opening in isolated mouse hearts. Am J Physiol Heart Circ Physiol 305: 16, 2013. 


\title{
A h mad Hanif
}

\author{
Curriculum Vitae
}

\section{Pharmaceutical and Pharmacological Sciences Department}

School of Pharmacy

West Virginia University

Robert C. Byrd Health Sciences Center

P.O. Box 9530

1 Medical Center Dr.

Morgantown, WV 26506-9530 - USA

Email: ahanif@mix.wvu.edu , dr_hanif75@hotmail.com

Phone: +1304 9069289

\section{Education:}

- 2011-2016: $\quad \mathrm{PhD}$

Department of Pharmaceutical and Pharmacological Sciences

West Virginia University

Morgantown, WV, USA 
- 1993 - 1998: $\quad$ B. Sc. Pharmacy

College of Pharmacy, University of Jordan

Amman, Jordan

Training Courses:

- “Customer Relationship Management” (CRM) by Hikma

- “Communication Skills” By CCM/TACK, Greece (Jan. 2004)

- “Negotiation Skills” By CCM/TACK, Greece (Mar. 2004)

- "Presentation Skills” By CCM/TACK, Greece (Oct. 2004)

- “Strategic Marketing in Action” By CCM/TACK, Greece (Aug. 2006)

- “Advanced Selling skills” By Merlin Training \& Development, Jordan (Oct. 2000)

- “Basic Selling skills” By Merlin Training \& Development, Jordan (June 1998)

\section{Qualifications and Work Experience:}

$2011-2016$

PhD in Pharmaceutical and Pharmacological Sciences

Research project: "Modulation of Coronary Reactive Hyperemia through Soluble Epoxide Hydrolase: Role of Oxylipins and PPAR $\gamma$ ”

Department of Basic Pharmaceutics Sciences, WVU

Morgantown, WV, USA

Sept. 2008 - June 2011: 


\section{Senior Product Manager}

In charge of training new recruits; oversaw an array of products' development and related marketing activities in the company's markets of UAE, Kuwait, Bahrain, Oman and Qatar

Marketing Department, Hikma Pharmaceuticals

Dubai, UAE

Jan. 2003 - Jan. 2007:

\section{Regional Product Manager}

In charge of training new recruits; oversaw an array of products' development and related marketing activities in the company's markets of Saudi Arabia, UAE, Bahrain, and Qatar Marketing Department, Hikma Pharmaceuticals

Riyadh, Saudi Arabia

June 2001 - Jan. 2003:

\section{Assistant Product Manager}

In charge of training new recruits; followed up products' launches and marketing activities

Marketing Department, APM Pharmaceuticals (became part of Hikma Group in 2007)

Riyadh, Saudi Arabia

June 1998 - May 2001:

\section{Medical Representative}


Detailed pharmaceutical drugs to target physicians to enhance their prescribing rate and ultimately product sales

APM Pharmaceuticals (became part of Hikma Group in 2007)

Amman, Jordan

February 1998 - June 1998:

\section{Pharmacist}

Dispensed medicines and counselled patients

Maram community pharmacy

Zarqa, Jordan

Teaching and Mentorship:

Spring 2016: Instructor: Biochemical Pharmacology 2 (PHAR 814), for PharmD students, School of Pharmacy, WVU, Morgantown, WV

Fall 2016: Instructor: Pharmacology / Cardiology (PHAR 824), for PharmD students, School of Pharmacy, WVU, Morgantown, WV

2012 - 2016: Trained graduate students and laboratory technicians the experimental techniques and procedures employed at our lab, such as the Langendorff perfused heart, Cardiac perfusate collection and preservation, organ bath, breeding and maintenance of animal colonies, and cell culture 
2001 -2011: Trained and taught company recruits the communication skills, medical and pharmaceutical backgrounds for the pharmaceutical items they detailed to target physicians.

\section{Experimental Skills:}

- Langendorff perfused mouse heart:

This technique included the euthanasia of the mouse, thoracotomy, heart isolation and preparation, hanging and retrograde perfusion, maintaining a beating heart for 2-3 hours, and recording cardiac function.

- Organ/tissue bath:

This technique included the euthanasia of the mouse, thoracotomy, aorta isolation and preparation, hanging and recording aortic response to different drugs.

- Cell culture:

This included the septic maintenance and passage of cell cultures, harvesting and freezing cell samples for analysis.

- Molecular techniques:

Accustomed to the common molecular techniques such as Western Blot, and PCR. 
- Computer programs and Powerlab system:

Well-acquainted with common computer programs, such as MS Word, Excel, PowerPoint. Also, has extensive experience with Powerlab System used to acquire and analyse data from Langendorff and Organ Bath experiments.

- Statistical software:

Well-familiarized with Prism-5 and MS-Excel Data Analysis software.

- Animal handling:

Worked on rodents (rats and mice) and certified by WVU's IACUC (Institutional Animal Care and Use Committee).

Awards and Honors:

2016: $\quad$ ASPET Graduate Student Travel Award at Experimental Biology 2016, San Diego, CA, USA

2014: Invited speaker for "12-Lipoxygenase and Disease: New Insights into Regulation and Inhibition of a Critical Enzyme" symposium organized by the American Society for Pharmacology and Experimental Therapeutics (ASPET) at Experimental Biology 14, San Diego, CA, USA 
2014: $\quad$ ASPET Graduate Student Travel Award at Experimental Biology 2014, San Diego, CA, USA

2014: WVU Graduate Student Travel Award by the Pharmaceutical and Pharmacological Sciences department to attend EB 14, San Diego, CA, USA

2014: $\quad$ WVU Dean's Travel Award to attend EB 14, San Diego, CA

2012: NIH Cardiovascular and Pulmonary Disease Training Grant (NHLBI-T32 PreDoctoral training grant) (selected for appointment)

Professional Membership:

2012: American Society for Pharmacology and Experimental Therapeutics (ASPET)

2012: The American Physiological Society (APS)

2006: National Association for the Boards of Pharmacy, USA

1998: Pharmaceutical Association, Jordan

Abstracts: National/International Conferences: 
1. "Increased Endothelial Expression of Human Soluble Epoxide Hydrolase Negatively Modulates Coronary Reactive Hyperemia in Isolated Mouse Heart”. Ahmad Hanif, Darryl C. Zeldin and Mohammed A. Nayeem. Presented at Experimental Biology Meeting 2015, Boston, MA

2. "Deletion of Soluble Epoxide Hydrolase Modulates Coronary Reactive Hyperemia in Isolated Mouse Hearts". Ahmad Hanif, Darryl C. Zeldin and Mohammed A. Nayeem. Presented at Experimental Biology Meeting 2015, Boston, MA

3. "Deletion of Soluble Epoxide Hydrolase Modulates Coronary Reactive Hyperemia in Isolated Mouse Hearts". Ahmad Hanif, Darryl C. Zeldin and Mohammed A. Nayeem. Presented at Experimental Biology Meeting 2014, San Diego, CA

4. "Inhibition of Soluble Epoxide Hydrolase by t-AUCB Modulates Coronary Reactive Hyperemia in Isolated Mouse Hearts". Ahmad Hanif, Christophe Morisseau and Mohammed A. Nayeem. Presented at Experimental Biology Meeting 2014, San Diego, CA

5. "Increased Endothelial Expression of Human Cytochrome P450 Epoxygenase 2J2 Positively Modulates Coronary Reactive Hyperemia in Isolated Mouse Heart”. Ahmad Hanif, Darryl C. Zeldin, John R. Falck and Mohammed A. Nayeem. Presented at Experimental Biology Meeting 2015, Boston, MA

\section{Publications:}


1. "Deletion of Soluble Epoxide Hydrolase Enhances Coronary Reactive Hyperemia in Isolated Mouse Heart: Role of Oxylipins and PPAR ”. Ahmad Hanif, Matthew L. Edin, Darryl C. Zeldin, Christophe Morisseau, and Mohammed A. Nayeem. (American Journal of Physiology-Regulatory, Integrative and Comparative Physiology, Am J Physiol Regul Integr Comp Physiol. 2016 Oct 1;311(4):R676-R688. doi: 10.1152/ajpregu.00237.2016).

2. "Effect of Soluble Epoxide Hydrolase Inhibition on Coronary Reactive Hyperemia: Role of Oxylipins and PPAR ". Ahmad Hanif, Matthew L. Edin, Darryl C. Zeldin, Christophe Morisseau, and Mohammed A. Nayeem. (PLoS One. 2016 Sep 1;11(9):e0162147. doi: 10.1371/journal.pone.0162147. eCollection 2016).

3. “Increased Endothelial Expression of Human Soluble Epoxide Hydrolase Negatively Modulates Coronary Reactive Hyperemia in Isolated Mouse Heart”. Ahmad Hanif, Darryl C. Zeldin and Mohammed A. Nayeem. (Under review).

Extra-Curricular Activities:

2014 - 2016: ISM Sunday school Principal: after volunteering as a teacher for a semester, I was elected principal for this 108-student Sunday school. I managed school curricula, recruiting teachers, student s evaluation, and furniture and disposables supplies. 
2014: Habitat for Humanity - Morgantown: volunteered to help in a number of humanitarian projects inside Morgantown, such as building houses for people who suffered homelessness due to natural disasters. 